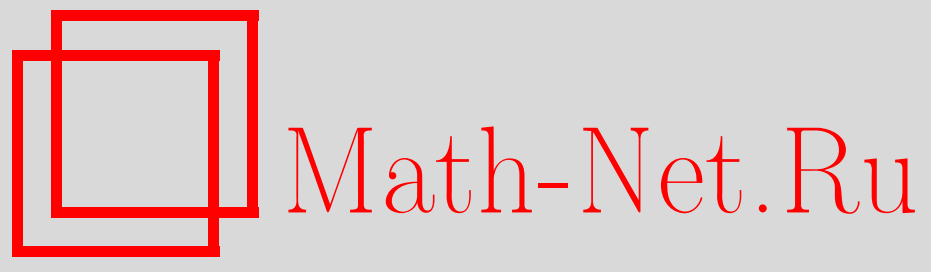

А. А. Шкаликов, Возмущения самосопряженных и нормальных операторов с дискретным спектром, УМH, 2016, том 71, выпуск 5, 113-174

DOI: https://doi.org/10.4213/rm9740

Использование Общероссийского математического портала Math-Net.Ru подразумевает, что вы прочитали и согласны с пользовательским соглашением http://www . mathnet.ru/rus/agreement

Параметры загрузки:

IP : 54.210 .77 .194

26 апреля 2023 г., 12:59:45

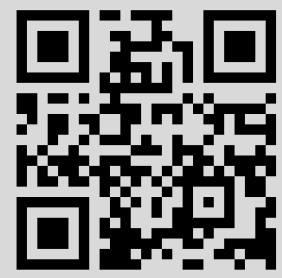




\title{
Возмущения самосопряженных и нормальных операторов с дискретным спектром
}

\begin{abstract}
А. А. Шкаликов
В статье изучаются спектральные свойства операторов вида $A=T+B$, где $B$ - несимметрический оператор, подчиненный самосопряженному или нормальному оператору $T$. Приводятся определения различных видов возмущений относительно оператора $T$ : вполне подчиненные, подчиненные с порядком $p<1$, локально подчиненные. Аналоги таких видов возмущений рассматриваются также для операторов, определяемых квадратичными формами. В случаях разных видов возмущений доказаны теоремы о сохранении свойств полноты корневых векторов операторов, о сохранении свойств базисности или безусловной базисности, о сравнении спектров операторов $T$ и $T+B$. Дается обзор работ по этой теме.

Библиография: 89 названий.

Ключевые слова: возмущения линейных операторов, оценки резольвенты, условия $p$-подчинения, условия локального подчинения, суммы квадратичных форм операторов, безусловные базисы, базисы Рисса, метод суммирования Абеля-Лидского.
\end{abstract}

DOI: $10.4213 / \mathrm{rm} 9740$

\section{СОДЕРЖАНИЕ}

1. Введение................................................

2. Подчиненные возмущения и оценки резольвенты возмущенных опера-

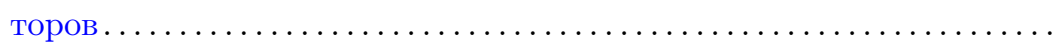

3. Подчиненные возмущения порядка $p<1$. Оценки резольвенты возмущенных операторов ............................... 122

4. Основная теорема об оценке резольвенты и теоремы о полноте ...... 125

5. Суммируемость спектральных разложений методом Абеля-Лидского.. 131

6. Теоремы о безусловной базисности ......................... 135

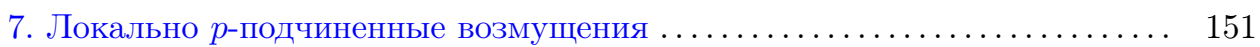

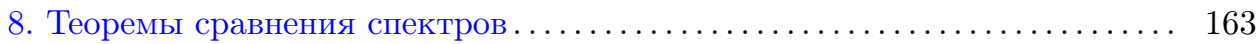

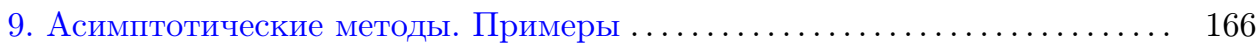

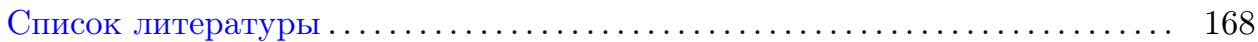

Работа выполнена при поддержке Российского научного фонда (проект № 14-11-00754).

(C) А. А. Шкаликов, 2016 


\section{1. Введение}

Пусть $T=T^{*}$ - самосопряженный неограниченный оператор в комплексном гильбертовом пространстве $H$ с областью определения $\mathscr{D}(T)$. Цель работы обсудить известные и получить новые результаты о спектральных свойствах оператора $A=T+B$, где $B$ - несимметрический оператор, в каком-то смысле подчиненный исходному оператору $T$. Мы рассмотрим различные формы подчинения, а в конце работы на конкретных и относительно простых примерах обсудим возможности применения полученных результатов и проведем сравнительный анализ различных методов.

При изложении основных результатов работы предполагается, что невозмущенный оператор $T$ имеет дискретный спектр. Его собственные значения далее обозначаются через $\left\{\mu_{k}\right\}$, а соответствующие нормированные собственные векторы - через $\left\{\varphi_{k}\right\}$. Система собственных векторов самосопряженного или нормального оператора $T$ образует ортонормированный базис в пространстве $H$, где определен оператор. Основные рассматриваемые ниже вопросы таковы: при каких возмущениях $B$ система собственных и присоединенных (корневых) векторов оператора $A=T+B$ остается полной, является базисом для суммирования методом Абеля-Лидского, является базисом или безусловным базисом? как различные типы возмущений влияют на асимптотику собственных значений?

Исследования на эту тему имеют давнюю историю. Как широкая самостоятельная тема в теории операторов она начала формироваться после выхода работ М. В. Келдыша [1]-[3]. Базой для этих работ послужили монография Я. Д. Тамаркина [4] и статья Т. Карлемана [5], хотя ни в одном из этих двух исследований нет каких-либо результатов по теории возмущений. В заметке [1] результаты были только анонсированы, а в статье [2] была проделана только подготовительная работа для получения теорем сравнения функций распределения спектров возмущенного и невозмущенного операторов. Доказательства анонсированных результатов (не всех) появились лишь спустя 20 лет в [3]. Конечно, в промежутке между выходом статей [1], [2] и статьи [3] публиковались работы с независимыми исследованиями - см., в частности, работы Ф. Браудера [6]-[8], М. С. Лифшица [9], Б. Р. Мукминова [10], И. М. Глазмана [11], М. Г. Крейна [12], В. Б. Лидского [13]-[15], А. С. Маркуса [16], [17], В.И. Мацаева [18]-[20], С. Агмона [21], [22], В.Э. Кацнельсона [23], [24]. Существенное влияние на развитие теории оказала монография И. Ц. Гохберга и М. Г. Крейна [25]. В последующие годы акцент сместился на исследования кратных разложений, т.е. исследования спектральных проблем пучков операторов (полиномов от спектрального параметра) и более общих оператор-функций от спектрального параметра. Вопросы спектральной теории стали связываться с проблемами разрешимости операторно-дифференциальных уравнений - отметим работы П. Д. Лакса [26] и С. Агмона и Л. Ниренберга [27]. В этой области возникли тонкие и глубокие проблемы, связанные с факторизацией пучков и оператор-функций, проблемы выделения части корневых векторов, которые отвечают множителям в факторизации. В последнее время эти проблемы зачастую коротко называют проблемой половины в спектральной теории. Но это самостоятельная очень широкая тема, тесно связанная с теорией самосопряженных и диссипативных операторов в пространстве с индефинитной метрикой. В этой статье мы затрагивать ее не будем. Частично 
с этими вопросами можно ознакомиться по книге [28] и статье [29]. Более поздних обзоров, достаточно широко охватывающих эту тему, по-видимому, нет.

При доказательстве многих из приведенных ниже результатов свойство самосопряженности невозмущенного оператора $T$ можно ослабить. А именно, достаточно требовать, чтобы $T$ был нормальным оператором, спектр которого локализован на конечном числе лучей комплексной плоскости или даже в некоторых параболических областях, примыкающих к этим лучам. На первый взгляд такое обобщение может показаться искусственным. Но оно оправданно, если иметь в виду приложения к спектральным задачам, зависящим полиномиально от спектрального параметра, т.е. к пучкам операторов. Чтобы понять, о чем идет речь, читатель может ознакомиться, например, с работами [3], [28]-[31].

Конечно, тема статьи является частью общей теории возмущений операторов, замечательное изложение которой имеется в монографии Т. Като [32]. Но в [32] почти не представлены результаты по теме этой статьи. Причина проста: в момент выхода первого издания книги Т. Като развитие этой тематики только начиналось - и начиналось с работ советских математиков, перевод которых на английский язык тогда не проводился. Однако в книге [25] эта тематика представлена как одна из основных, хотя излагалась она в рамках теории ограниченных операторов, что затрудняло непосредственное использование результатов для дифференциальных операторов, связанных с конкретными задачами механики. После выхода книги [25] тематике настоящей статьи было посвящено очень большое число работ, относящихся как к общим, так и к конкретным дифференциальным операторам. Частичный обзор библиографии и конкретных задач, к которым результаты этой статьи могут быть применены, имеется в статьях [33]--[35], обзоре [36], и книгах [37] и [38]. Здесь мы не стремимся охватить широкий спектр работ. Главная наша цель - описать основные идеи и методы, связанные с результатами, которые мы считаем наиболее важными. Имеющиеся в литературе полные доказательства относящихся к теме результатов часто технически сложны, и их детальный разбор - нелегкая задача для читателя. Поэтому, где возможно, мы даем полные доказательства или проводим доказательство для наиболее важных частных случаев.

Побудительным мотивом для написания этой статьи явились работы последних лет, выполненные Б. С. Митягиным, Дж. Аддучи, П. Джаковым и П. Зиглом - см. [39]-[50]. В изложении используются результаты автора [51], [52], некоторые из которых были анонсированы в более частном виде.

Автор благодарит М. С. Аграновича, М. М. Маламуда, Б. С. Митягина и А. М. Савчука, которые ознакомились со статьей и сделали ряд замечаний.

\section{2. Подчиненные возмущения и оценки резольвенты возмущенных операторов}

Пусть $T$ - замкнутый, плотно определенный оператор в гильбертовом пространстве $H$; через $\mathscr{D}(T)$ обозначаем область его определения, а через $\sigma(T)$ и $\rho(T)$ - его спектр и резольвентное множество соответственно. Если $T$ замкнут, а $\mathscr{D}(T)$ плотна, то однозначно определен сопряженный оператор $T^{*}$, который обладает теми же свойствами, при этом $T=T^{* *}$. Оператор $T=T^{* *}$ называется нормальным, если

$$
T^{*} T=T T^{*} .
$$


Согласно теореме Дж. фон Неймана (см. [32; гл. 5, § 3.7]) оба оператора в этом равенстве самосопряженные. Из определения получаем $[32 ;$ гл. $5, \S 3.8]$, что $\mathscr{D}(T)=\mathscr{D}\left(T^{*}\right)$ и $\|T x\|=\left\|T^{*} x\right\|$ для $x \in \mathscr{D}(T)$. Если $\operatorname{Ker} T=\{0\}$, то определенный на плотном множестве оператор $T^{*} T^{-1}$ продолжается по непрерывности на все $H$ как унитарный. Для нормальных операторов, так же как для самосопряженных, справедлива спектральная теорема, которую можно формулировать как в терминах функционального исчисления, так и в терминах оператора умножения или в терминах интегрального представления с помощью семейства ортогональных проекторов [53; гл. 10, 14]. В случае компактного вложения $\mathscr{D}(T) \hookrightarrow H$ нормальный оператор $T$ имеет дискретный спектр и допускает представление

$$
T=\sum_{k} \mu_{k}\left(\cdot, \varphi_{k}\right) \varphi_{k}, \quad \mathscr{D}(T)=\left\{x \in H: \sum_{k}\left|\mu_{k}\left(x, \varphi_{k}\right)\right|^{2}<\infty\right\},
$$

где $\left\{\varphi_{k}\right\}$ - полная ортонормированная система собственных функций, отвечающих собственным значениям $\mu_{k}$, имеющим предельную точку только в $\infty$. Далее мы будем использовать следствие спектральной теоремы (очевидное в случае дискретного спектра): если $0, \lambda \notin \rho(T)$, то

$$
\left\|T^{\alpha}(T-\lambda)^{-\beta}\right\|=\sup _{t \in \sigma(T)} \frac{|t|^{\alpha}}{|t-\lambda|^{\beta}}, \quad \alpha, \beta \geqslant 0
$$

где $T^{\alpha}=\sum \mu_{k}^{\alpha}\left(\cdot \varphi_{k}\right) \varphi_{k}$ и для функции $\mu^{\alpha}$ биксируется подходящая ветвь корня.

Оператор $B$ называется $T$-подчиненным (или $T$-ограниченным), если $\mathscr{D}(B) \supset$ $\mathscr{D}(T)$ и найдутся постоянные $b$ и $M$ такие, что

$$
\|B x\| \leqslant b\|T x\|+M\|x\| \quad \forall x \in \mathscr{D}(T) .
$$

Нижняя грань $b^{\prime}$ постоянных $b$, при которых выполняется оценка $(2.2)$ с постоянной $M=M(b)$, называется $T$-гранъю оператора $B$. В случае $b^{\prime}=0$ оператор $B$ назовем вполне $T$-подчиненным.

Сложнее определить $T$-подчиненность в смысле квадратичных форм, которая будет важна в дальнейшем. Обычно в литературе определяют это понятие в случае самосопряженного равномерно положительного оператора $T$. В этом случае оператор $T$ определяется своей квадратичной формой $\mathfrak{t}[x, x]=$ $\left(T^{1 / 2} x, T^{1 / 2} x\right)$ на области $\mathscr{D}(\mathfrak{t})=\mathscr{D}\left(T^{1 / 2}\right)$. Предположим, что задана форма $\mathfrak{b}=\mathfrak{b}[x, x]$ такая, что

$$
|\mathfrak{b}[x, x]| \leqslant b \mathfrak{t}[x, x]+M\|x\|^{2}, \quad \mathscr{D}(\mathfrak{b}) \supset \mathscr{D}(\mathfrak{t}),
$$

с некоторыми постоянными $b<1, M>0$. Тогда сумма форм $\mathfrak{t}+\mathfrak{b}$ оказывается $m$-секториальной и замкнутой, а значит, однозначно определяет некоторый $m$-секториальный оператор, который обозначаем через $T+B$. Для этого оператора справедлива первая теорема о представлении [32; гл. 6, §3.1]. Однако такой подход слишком ограничителен - с его помощью нельзя определить сумму форм, например, в случае самосопряженного невозмущенного оператора $T$, 
если этот оператор не является полуограниченным. Для случая $T=T^{*}$ и подчиненности формы $\mathfrak{b}$ оператору $T$ с порядком $p<1$ (см. раздел 3 ) определение суммы $T+B$ было предложено А. С. Маркусом и В. И. Мацаевым [54]. Аналогичные аргументы использовали П. Джаков и Б. С. Митягин [45] при исследовании оператора Дирака с комплексным потенциалом. В настоящей статье мы предлагаем более общий подход.

Оператор $T$ называется позитивным, если отрицательный луч $(-\infty, 0]$ принадлежит резольвентному множеству $T$ и выполняется оценка

$$
\left\|(T-\lambda)^{-1}\right\| \leqslant \frac{C}{|\lambda|+1} \quad \forall \lambda \leqslant 0 .
$$

Известно [55; гл. 4, § 14], что для позитивных операторов корректно определены дробные степени оператора $T$, являющиеся замкнутыми операторами в пространстве $H$. При умножении степеней $T^{\alpha}$ сохраняются обычные правила сложения показателей. Справедливо также равенство $\left(T^{\alpha}\right)^{*}=\left(T^{*}\right)^{\alpha}$. В частности, однозначно определен квадратный корень $T^{1 / 2}$, который является замкнутым оператором, при этом его спектр находится в угле $|\arg \lambda|<\pi / 2$, а оценка $(2.4)$ для $T^{1 / 2}$ выполняется с другой константой во всей левой полуплоскости.

Гильбертово пространство с нормой графика

$$
\|x\|_{1}^{2}=\left\|T^{1 / 2} x\right\|^{2}+\|x\|^{2}
$$

обозначим через $H_{1}$, а дуальное к нему по отношению к скалярному произведению в $H$ обозначим через $H_{-1}$. Согласно условию (2.4) оператор $T$ обратим. По теореме о полярном представлении $[32 ;$ гл. $6, \S 2.7]$ имеем $T^{1 / 2}=U R$, где $U$ - унитарный оператор в $H$, а $R=\left(\left(T^{*}\right)^{1 / 2} T^{1 / 2}\right)^{1 / 2}=:\left|T^{1 / 2}\right|$. Из определения следует, что операторы

$$
T^{1 / 2}: H_{1} \rightarrow H, \quad R: H_{1} \rightarrow H, \quad R: H \rightarrow H_{-1}
$$

ограничены и обратимы, т. е. биективны. Далее будем предполагать выполненным условие

$$
\mathscr{D}\left(\left(T^{*}\right)^{1 / 2}\right)=\mathscr{D}\left(T^{1 / 2}\right) .
$$

Вопрос о том, выполняется ли это условие для позитивных операторов - известная проблема, которую называют проблемой Като для квадратного корня (Kato square root problem). Она отрицательно решена: имеются позитивные и даже $m$-секториальные операторы, для которых равенство (2.6) нарушается. Но это условие выполняется для широкого класса как общих, так и дифференциальных операторов. Литература и эффективные достаточные условия имеются, например, в статье М. С. Аграновича и А. М. Селицкого [56]. Конечно, для нормальных операторов это равенство выполняется.

Квадратичную форму $\mathfrak{b}=\mathfrak{b}[x, x]$ назовем $T$-подчиненной, если $\mathscr{D}(\mathfrak{b}) \supset \mathscr{D}\left(T^{1 / 2}\right)$ и выполнено неравенство (2.3), где

$$
\mathfrak{t}[x, x]=\left(T^{1 / 2} x, T^{1 / 2} x\right)=\|R x\|^{2},
$$

а $M$ и $b-$ постоянные. 
Теорема 2.1. Пусть форма $\mathfrak{b}$ является Т-подчиненной и выполнено условие (2.6). Тогда существует ограниченный оператор $B: H_{1} \rightarrow H_{-1}$ такой, что

$$
\mathfrak{b}[x, x]=(B x, x) \quad \forall x \in \mathscr{D}\left(T^{1 / 2}\right),
$$

а оператор $T$ продолжается по непрерывности как ограниченный оператор из $H_{1}$ в $H_{-1}$. Тем самым, сумма форм $(T x, x)+\mathfrak{b}[x, x]$, определенная при $x \in \mathscr{D}(T)$, представляется в виде

$$
((T+B) x, x), \quad \text { әде } T+B: H_{1} \rightarrow H_{-1} \text { ограничен. }
$$

ДокАЗАтЕльство. Заметим, что если форма $\mathfrak{b}$ определена на $H_{1}$ и выполняется оценка (2.3), то для квадратичной формы

$$
\widehat{\mathfrak{b}}[y, y]=\mathfrak{b}\left[T^{-1 / 2} y, T^{-1 / 2} y\right], \quad y \in H,
$$

получаем оценку

$$
|\widehat{\mathfrak{b}}[y, y]| \leqslant b\|y\|^{2}+M\left\|T^{-1 / 2} y\right\|^{2} \leqslant C\|y\|^{2}, \quad C=\text { const } .
$$

Поэтому форма $\widehat{\mathfrak{b}}$ однозначно определяет ограниченный оператор $\widehat{B}: H \rightarrow H$ такой, что

$$
\mathfrak{b}[x, x]=\left(\widehat{B} T^{1 / 2} x, T^{1 / 2} x\right)=\left(\left(T^{*}\right)^{1 / 2} \widehat{B} T^{1 / 2} x, x\right), \quad x \in H_{1}
$$

(здесь $\left.\left(T^{*}\right)^{1 / 2}=\left(T^{1 / 2}\right)^{*}=(U R)^{*}=R U^{*}\right)$. Поэтому из $(2.5)$ следует, что этот оператор допускает продолжение по непрерывности как ограниченный оператор из $H$ в $H_{-1}$. Следовательно, форма $\mathfrak{b}$ однозначно представляется ограниченным оператором $B=\left(T^{*}\right)^{1 / 2} \widehat{B} T^{1 / 2}: H_{1} \rightarrow H_{-1}$.

При выполнении условия (2.6) выполнено равенство $\mathscr{D}\left(\left(T^{*}\right)^{1 / 2}\right)=H_{1}$. Поэтому оператор $\left(T^{*}\right)^{1 / 2}:=H_{1} \rightarrow H$ биективен. Поскольку $R: H_{1} \rightarrow H$ биективен, то

$$
\left(T^{*}\right)^{1 / 2}=F R, \quad T^{1 / 2}=R F^{*}, \quad \text { где } F: H \rightarrow H \text { биективен. }
$$

Но тогда оператор $T=T^{1 / 2} T^{1 / 2}=R F^{*} T^{1 / 2}: H_{1} \rightarrow H_{-1}$ также биективен. Теорема доказана.

Мы получили операторную реализацию суммы форм, но для получения информации о спектре этого оператора нужна дальнейшая работа. Для сужения оператора $T+B$ на пространство $H$ с областью

$$
\mathscr{D}(T+B)=\left\{x \in H_{1}:(T+B) x \in H\right\}
$$

будем использовать то же обозначение $T+B$. Отметим, что если оператор $T+B: H_{1} \rightarrow H_{-1}$ есть биекция, то $T+B$ является замкнутым ограниченно обратимым оператором в $H$ (так как его образ совпадает со всем пространством). Однако область $(2.8)$ не совпадает с $\mathscr{D}(T)$, если условие $\mathscr{D}(B) \supset \mathscr{D}(T)$ не выполнено. В общем случае (когда нет биективности), оператор $T+B$ может быть даже не замыкаемым в пространстве $H$. Мы получим в этом разделе 
эффективные достаточные условия для корректной определенности оператоpa $T+B$.

Форму $\mathfrak{b}$ будем называть вполне $T$-подчиненной, если нижняя грань постоянных $b$, при которых выполняется неравенство (2.3), равна нулю. При этом всегда (в том числе и в формулировках утверждений) будем предполагать, что оператор $T$ позитивный и выполняется условие (2.6). Понятие вполне подчиненности связано с компактностью оператора $B T^{-1}$ и оператора $\widehat{B}$, порожденного формой (2.7).

ПреДЛОЖениЕ 2.2. Если оператор $B T^{-1}$ компактен, то оператор В являтся вполне Т-подчиненным. Аналогично, если оператор $\widehat{B}$, порожденный формой (2.7), компактен, то форма $\mathfrak{b}$ является вполне T-подчиненной.

ДокАзАтельство. Пусть оператор $K=B T^{-1}$ компактен в $H$. Тогда его можно приблизить (см., например, $[57 ;$ гл. $5, \S 85])$ по операторной норме конечномерными операторами, т. е.

$$
K=\sum_{k=1}^{n}\left(\cdot, x_{k}\right) y_{k}+K_{\varepsilon}, \quad\left\|K_{\varepsilon}\right\| \leqslant \varepsilon .
$$

Из этого представления получаем, что достаточно доказать утверждение для одномерного оператора $K=(\cdot x) y$. Область $\mathscr{D}\left(T^{*}\right)=\operatorname{Im}\left(T^{*}\right)^{-1}$ является плотным в $H$ множеством, поэтому достаточно доказать утверждение для такого оператора при условии $\psi \in \mathscr{D}\left(T^{*}\right)$, т. е. для $\psi=\left(T^{*}\right)^{-1} z, z \in H$. Но для одномерных операторов такого вида имеем $\|K T x\| \leqslant\|z\|\|\varphi\|\|x\|$, т. е. оценка $(2.2)$ выполняется при $b=0$.

Доказательство для форм проводится аналогично. Предложение доказано.

ЗАмечание 2.3. Если оператор $B$ вполне $T$-подчинен, то оператор $K=$ $B T^{-1}$ может не быть компактным. Однако если $T^{-1}$ компактен (это эквивалентно компактности вложения $\mathscr{D}(T) \hookrightarrow H$ ), то обратное к предложению 2.2 утверждение верно: полная $T$-подчиненность оператора $B$ влечет компактность оператора $K=B T^{-1}$. Действительно, положим $T^{-1}=\sum_{k=1}^{n}\left(\cdot, x_{k}\right) y_{k}+R_{\varepsilon}$, где $\left\|R_{\varepsilon}\right\| \leqslant \varepsilon$. Из оценки (2.2), полагая в ней $b=\varepsilon$, получаем неравенство $\|K x\| \leqslant 2 \varepsilon$ для всех $x$, принадлежащих подпространству конечной коразмерности в $H$. Тогда компактность оператора $K$ следует из определения. Доказательство для форм получается аналогичным образом.

Отметим еще, что в случае компактного вложения $\mathscr{D}(T) \hookrightarrow H$ вполне подчиненные возмущения называют относительно компактными возмущениями.

Пусть $\Gamma_{\varphi}=\{\lambda \in \mathbb{C}: \arg \lambda=\varphi\}-$ луч в комплексной плоскости $\mathbb{C}$ с началом в нуле. Очевидно, что резольвента самосопряженного оператора $T$ на лучах $\Gamma_{\varphi}$ при $\varphi \neq 0, \pi$ подчинена оценке

$$
\left\|(T-\lambda)^{-1}\right\| \leqslant C|\lambda|^{-1}, \quad|\lambda|>r_{0},
$$

где $r_{0}=0$ и $C=|\sin \varphi|^{-1}$. Резольвента любого оператора не может при $\lambda \rightarrow \infty$ убывать быстрее, более того, $C=1$ является наилучшей возможной константой для любого луча. Поэтому разумно говорить, что луч $\Gamma_{\varphi}$ является лучом 
наилучшего убывания резольвенты, если на этом луче асимптотически (при достаточно больших $r_{0}$ ) выполняется оценка $(2.9)$. Очевидно, что $\Gamma_{\varphi}$ является лучом наилучшего убывания резольвенты $T$ тогда и только тогда, когда оператор $-e^{-i \varphi} T+c$ является позитивным при достаточно большом $c$.

ЗАмЕчАНИЕ 2.4. Если луч $\Gamma_{\varphi}$ является лучом наилучшего убывания резольвенты оператора $T$, то найдется малый сектор $\Lambda_{\delta}(\varphi)=\{\lambda \in \mathbb{C}:|\arg \lambda-\varphi|<\delta\}$, в котором оценка (2.9) выполняется с некоторой другой константой $C$. Действительно, положим $\mu=e^{i \tau} \lambda$. Тогда

$$
(T-\mu)^{-1}=(T-\lambda)^{-1}[1+R(\mu)]^{-1}, \quad R(\mu)=\left(1-e^{i \tau}\right) \lambda(T-\lambda)^{-1},
$$

причем $\|R(\mu)\|<1 / 2$, если $\tau \leqslant \delta$ и $\left|e^{i \delta}-1\right| C<1 / 2$, где $C$ - постоянная из $(2.9)$. Следовательно, для всех $\lambda \in \Lambda_{\delta}(\varphi)$ верна оценка (2.9) с удвоенной константой $C$. Поэтому если $T$ обратим, обладает дискретным спектром и имеет луч наилучшей оценки резольвенты, то, не ограничивая общности, можно считать, что этот луч не проходит через собственные значения, а потому, с точностью до умножения на скаляр, оператор $T$ является позитивным.

Теорема 2.5. Пусть $\Gamma_{\varphi}-$ луч наилучшего убывания резолъвенты оператора T. Если оператор В вполне T-подчинен, то этот же луч является лучом наилучшего убывания резольвенты возмущенного оператора $A=T+B$. Пусть $T$ - нормальный и позитивный оператор с точностъю до умножения на скаляр $e^{i \varphi}$, a $\mathfrak{t}$ - квадратичная форма, отвечающая этому оператору. Если квадратичная форма $\mathfrak{b}$ является вполне T-подчиненной, то порождаемый квадратичной формой $\mathfrak{t}+\mathfrak{b}$ оператор $T+B$ с областъю (2.8) является замкнутым оператором в $H$, а $\Gamma_{\varphi}$ является лучом наилучшего убывания его резольвенты.

ДокАзАтЕльство. Первое утверждение получается просто. Пусть $B$ вполне $T$-подчиненный оператор. Тогда выполнена оценка (2.2) с произвольно малым числом $b$. На луче $\Gamma_{\varphi}$ имеем

$$
(T+B-\lambda)^{-1}=(T-\lambda)^{-1}(1+S(\lambda))^{-1}, \quad S(\lambda)=B(T-\lambda)^{-1} .
$$

Далее, в силу равенства $T(T-\lambda)^{-1}=1+\lambda(T-\lambda)^{-1}$ имеем

$$
\|S(\lambda) x\| \leqslant b\left\|T(T-\lambda)^{-1} x\right\|+M\left\|(T-\lambda)^{-1} x\right\| \leqslant b(1+C)+M C|\lambda|^{-1}<\frac{1}{2}\|x\|,
$$

если взять $b$ достаточно малым, а $|\lambda|$ достаточно большим. Тогда из (2.10) получаем нужное утверждение.

Теперь пусть оператор $T$ нормальный ${ }^{1}$ и позитивный (не ограничивая общности, считаем, что луч $\Gamma_{\varphi}$ совпадает с $\left.\mathbb{R}_{-}\right)$. Тогда при $\lambda \leqslant 0$ корректно определены квадратные корни $(T-\lambda)^{-1 / 2}$. Справедливо равенство

$$
(T+B-\lambda)=(T-\lambda)^{1 / 2}\left(1+S_{1}(\lambda)\right)(T-\lambda)^{1 / 2},
$$

\footnotetext{
${ }^{1} \mathrm{~B}$ действительности утверждение теоремы 2.5 сохраняет силу не только для нормальных, но и для произвольных позитивных операторов, для которых выполнено условие (2.6). Доказательство будет представлено в другой работе.
} 
где $S_{1}(\lambda)=(T-\lambda)^{-1 / 2} B(T-\lambda)^{-1 / 2}$. Положим

$$
\begin{gathered}
V(\lambda)=(T-\lambda)^{-1 / 2}, \quad S_{2}(\lambda)=V(\lambda)^{*} B V(\lambda)=U(\lambda) S_{1}(\lambda), \\
U(\lambda)=V(\lambda)^{*} V^{-1}(\lambda) .
\end{gathered}
$$

Поскольку $T$ нормальный, то $U(\lambda)$ - унитарный оператор, а потому $\left\|S_{1}(\lambda) x\right\|=$ $\left\|S_{2}(\lambda) x\right\|$. Далее,

$$
\left|\left(S_{2}(\lambda) x, x\right)\right|=|(B V(\lambda) x, V(\lambda) x)| \leqslant b\left\|T^{1 / 2} V(\lambda) x\right\|^{2}+M\|V(\lambda) x\|^{2} .
$$

Согласно (2.1), на луче $\mathbb{R}_{-}$получаем оценки

$$
\left\|T^{1 / 2}(T-\lambda)^{-1 / 2}\right\| \leqslant C, \quad\left\|(T-\lambda)^{-1 / 2}\right\| \leqslant C(|\lambda|+1)^{-1 / 2}, \quad \lambda \in \mathbb{R}_{-},
$$

где постоянная $C$ зависит только от раствора угла, в котором нет точек спектра оператора $T$. Поэтому первое слагаемое в правой части неравенства $(2.14)$ ограничено числом $b C\|x\|^{2}$, не превосходящим $\|x\|^{2} / 4$, если $b$ взять достаточно малым, а второе слагаемое оценивается сверху величиной $M C(|\lambda|+1)^{-1}\|x\|^{2}<$ $\|x\|^{2} / 4$, если $|\lambda|>r_{0} \gg 1$. Следовательно, $\left\|S_{2}(\lambda)\right\|=\left\|S_{1}(\lambda)\right\|<1 / 2$ при достаточно больших $|\lambda|$. Но тогда

$$
\begin{gathered}
(T+B-\lambda)^{-1}=(T-\lambda)^{-1 / 2}\left(1+S_{1}(\lambda)\right)^{-1}(T-\lambda)^{-1 / 2}, \\
\left\|(T+B-\lambda)^{-1}\right\| \leqslant 2\left\|(T-\lambda)^{-1 / 2}\right\|^{2} \leqslant 2 C(1+|\lambda|)^{-1} .
\end{gathered}
$$

Это завершает доказательство теоремы.

Отметим, что представление (2.12) выражает сущность проблемы. Сумме форм $\mathfrak{t}+\mathfrak{b}$ мы ставим в соответствие оператор

$$
T+B=\lambda+(T-\lambda)^{1 / 2}\left(1+S_{1}(\lambda)\right)(T-\lambda)^{1 / 2},
$$

где $S_{1}=U^{-1} S_{2}$, оператор $U$ унитарный, а оператор $S_{2}$ порождается ограниченной квадратичной формой. Оператор $T+B$ в пространстве $H$ может оказаться "плохим" (т. е. даже незамыкаемым). Но в случае $\left\|S_{1}\right\|<1$ оператор $T+B-\lambda$ с областью (2.8) замкнут, так как корректно определен обратный оператор, выражаемый равенством (2.15).

ПреДЛОЖЕНИЕ 2.6. Пусть Т - нормальный оператор, а его спектр в некотором секторе

$$
\Lambda_{\alpha}=\left\{\lambda \in \mathbb{C}:|\arg \lambda| \leqslant \frac{\pi \alpha}{2}\right\}, \quad \alpha<2,
$$

расположен на положительной полуоси. Если $B$ вполне Т-подчинен, то при любом $\varepsilon>0$ и достаточно большом $r_{0}$ область

$$
\Omega_{\varepsilon}=\left\{\lambda \in \Lambda_{\alpha-\varepsilon} \backslash \Lambda_{\varepsilon},|\lambda|>r_{0}\right\}
$$

не содержит точек спектра оператора $A=T+B$ и для всех $\lambda \in \Omega_{\varepsilon}$ справедлива оценка (2.9). Аналогичное утверждение справедливо для оператора $T+B$, если оператор $B$ порожден вполне $T$-подчиненной формой. 
ДокАЗАТЕЛьство. Это утверждение является следствием теоремы 2.5 и замечания 2.4 .

ЗАмечАниЕ 2.7. В случае компактности вложения $\mathscr{D}(T) \hookrightarrow H$ оператор $T^{-1}$ компактен, т. е. оператор $T$ имеет дискретный спектр. Для вполне $T$-подчиненных возмущений (обоих типов) резольвентное множество $\rho(T+B)$ при наших условиях на оператор $T$ непусто, а оператор $(T+B-\lambda)^{-1}$ при $\lambda \in \rho(T+B)$ компактен. Поэтому спектры возмущенных операторов также дискретны.

\section{3. Подчиненные возмущения порядка $p<1$. Оценки резольвенты возмущенных операторов}

В этом разделе мы предполагаем, что невозмущенный оператор $T$ является нормальным и позитивным с точностью до умножения на скаляр. В частности, $T$ обратим и корректно определены его дробные степени $T^{p}$. В литературе рассматривались разные типы возмущений, более сильные, нежели вполне $T$-подчиненные. Пусть $0 \leqslant p<1$. Будем говорить, что оператор $B$ сильно $p$-подчинен оператору $T$, если он $T^{p}$-подчинен при некотором $p<1$. С учетом обратимости оператора $T$ это эквивалентно оценке

$$
\left\|B T^{-p} x\right\| \leqslant b\|x\| \quad \forall x \in \mathscr{D}(T), \quad b=\text { const },
$$

т. е. ограниченности оператора $B T^{-p}$. Наряду с этим условием рассмотрим условие

$$
\|B x\| \leqslant b\|T x\|^{p}\|x\|^{1-p} \quad \forall x \in \mathscr{D}(T) .
$$

При его выполнении оператор $B$ назовем $p$-подчиненным оператору $T$. Известно [55; теорема 12.2], что условие (3.1) влечет (3.2). Обратное утверждение неверно (этот факт доказан в [58]). Однако если $B$ является $p$-подчиненным, то он $T^{q}$-подчинен при любом $q>p$ (см. [55; теорема 12.3]).

Чтобы дать более точные формулировки последующих теорем, неравенство (3.2) запишем при $p>0$ в модифицированном виде

$$
\|B x\| \leqslant b\|T x\|^{p}\|x\|^{1-p}+M\|x\| \quad \forall x \in \mathscr{D}(T) .
$$

Нижнюю грань постоянных $b$, при которых выполнено это неравенство с некоторыми постоянными $M=M(b)$, назовем точной гранъю $p$-подчиненности.

Теперь определим $p$-подчиненность в смысле форм. Зафиксируем $p \in[0,1)$. Будем говорить, что форма $\mathfrak{b}=\mathfrak{b}[x, x]$ силъно p-подчинена оператору $T$, если она является $T^{p}$-подчиненной. С учетом обратимости оператора $T$ это эквивалентно неравенству

$$
|\mathfrak{b}[x, x]| \leqslant b\left\|T^{p / 2} x\right\|^{2} \quad \forall x \in \mathscr{D}\left(T^{p / 2}\right) .
$$

Известно [59; гл. 1.15], что $\mathscr{D}\left(T^{\theta}\right)=H_{\theta}$ при $\theta \in[0,1 / 2)$. Здесь $H_{\theta}=\left[H_{1}, H\right]_{\theta}-$ интерполяционные пространства, $H_{1}=\mathscr{D}\left(T^{1 / 2}\right)$, с нормой графика. Тогда условие (3.4) эквивалентно ограниченности оператора $B: H_{p} \rightarrow H_{-p}$, порожденного формой $\mathfrak{b}$ (см. теорему 2.1), где $H_{-p}$ - дуальное к $H_{p}$ пространство. 
В работе [54] введено следующее условие:

$$
|\mathfrak{b}[x, x]| \leqslant b\left\|T^{1 / 2} x\right\|^{2 p}\|x\|^{2-2 p} \quad \forall x \in \mathscr{D}\left(T^{1 / 2}\right) .
$$

В этом случае форму $\mathfrak{b}$ будем называть $p$-подчиненной оператору $T, 0 \leqslant p<1$. Нетрудно видеть, что это условие слабее условия (3.4). При его выполнении в [54] получены теоремы сравнения функций распределения спектров операторов $T$ и $T+B$ (здесь и далее сумму $T+B$ мы понимаем так, как это определено в теореме 2.1). Теоремы о безусловной базисности и базисности для суммирования методом Абеля-Лидского при выполнении условия (3.5) и в предположении $T \gg 0$ получил М. С. Агранович [60].

При $p>0$ условие (3.5) также запишем в более точном виде

$$
|\mathfrak{b}[x, x]| \leqslant b\left\|T^{1 / 2} x\right\|^{2 p}\|x\|^{2-2 p}+M\|x\|^{2} \quad \forall x \in \mathscr{D}\left(T^{1 / 2}\right) .
$$

Нижнюю грань постоянных $b$, при которых выполнено это неравенство с некоторыми постоянными $M=M(b)$, назовем точной гранъю $p$-подчиненности.

Докажем утверждения о локализации спектра оператора $T+B$ при $p$-подчиненных возмущениях.

ПреДЛОЖеНИЕ 3.1. Пусть T - нормалъный оператор $u$, так же как в предложении 2.6, $\sigma(T) \cap \Lambda_{\alpha} \subset \mathbb{R}_{+}$, где сектор $\Lambda_{\alpha}$ определен формулой (2.16). Рассмотрим параболические области (при $p=0$ полуполосы)

$$
\Pi_{p, h}=\left\{\lambda=\sigma+i \tau \in \mathbb{C}: \sigma \geqslant 0,|\tau| \leqslant h \sigma^{p}\right\} .
$$

Если оператор $B$ является р-подчиненным с точной гранъю $b^{\prime}$, то спектр опеpатора $A=T+B$, расположенный внутри вдвое меньшего сектора $\Lambda_{\alpha / 2}$, асимптотически локализован в области $\Pi_{p, h}$, если $h>b^{\prime}$, а в области $\Lambda_{\alpha / 2} \backslash \Pi_{p, h}$ при любом $\varepsilon>0$ асимптотически выполняется оценка

$$
\left\|(A-\lambda)^{-1}\right\| \leqslant\left(\frac{b^{\prime}+\varepsilon}{h-b^{\prime}}\right) \frac{1}{|\tau|}, \quad|\lambda| \gg 1 .
$$

ДокаЗАтеЛЬСтво (ср. [58]). Положим $S(\lambda)=B(T-\lambda)^{-1}$. Тогда

$$
(A-\lambda)^{-1}=(T-\lambda)^{-1}(1+S(\lambda))^{-1} .
$$

Из условия (3.3) следует, что

$$
\|S(\lambda)\| \leqslant b\left\|T(T-\lambda)^{-1}\right\|^{p}\left\|(T-\lambda)^{-1}\right\|^{1-p}+M\left\|(T-\lambda)^{-1}\right\| .
$$

Из оценки (2.1) получаем

$$
\left\|T(T-\lambda)^{-1}\right\|=\sup _{t \in \sigma(T)}\left|\frac{t}{t-\lambda}\right|=\inf _{t \in \mathbb{R}_{+}}\left|1-\frac{\lambda}{t}\right| \leqslant \frac{|\lambda|}{\tau}, \quad \lambda=\eta+i \tau,
$$

так как

$$
\left(1-\frac{\lambda}{t}\right)^{2}=1-\frac{2 \eta}{t}+\frac{|\lambda|^{2}}{t^{2}}=\left(\frac{|\lambda|}{t}-\frac{\eta}{|\lambda|}\right)^{2}+\frac{\tau^{2}}{|\lambda|^{2}} \geqslant \frac{\tau^{2}}{|\lambda|^{2}}
$$


Здесь мы учитываем, что спектр оператора $T$, расположенный вне сектора $\Lambda_{\alpha}$, на оценки внутри сектора $\Lambda_{\alpha / 2}$ не влияет. Из полученных оценок следует, что

$$
\|S(\lambda)\| \leqslant b\left(\frac{|\lambda|}{|\tau|}\right)^{p}\left(\frac{1}{|\tau|}\right)^{1-p}+\frac{M}{|\tau|} \leqslant b \frac{|\lambda|^{p}}{|\tau|}(1+o(1)) .
$$

Здесь $o(1)=O\left(|\lambda|^{-p}\right) \rightarrow 0$ при $|\lambda| \rightarrow \infty$ (мы предполагаем, что $p>0$; при $p=0$ имеем $o(1)=0)$. В секторе $\Lambda_{\alpha / 2}$ верна очевидная оценка $\left\|(T-\lambda)^{-1}\right\| \leqslant|\tau|^{-1}$. Поэтому в этом секторе при $|\tau| \geqslant h|\lambda|^{p}, h>b$ (т. е. вне $\Pi_{p, h}$ ), получаем

$$
\|S(\lambda)\| \leqslant \frac{b}{h}(1+o(1)), \quad\left\|(1+S(\lambda))^{-1}\right\| \leqslant \frac{b}{h-b}<\frac{b^{\prime}+\varepsilon}{h-b^{\prime}}, \quad|\lambda| \gg 1 .
$$

Здесь число $\varepsilon$ может быть выбрано произвольно малым, так как число $b$ может быть выбрано произвольно близким к $b^{\prime}$. При $|\tau|=\eta^{p}$ имеем $|\lambda| \sim \eta$, и, следовательно, кривая $|\tau|=h|\lambda|^{p}$ асимптотически совпадает с границей параболической области $\Pi_{p, h}$. Поэтому последняя оценка остается справедливой вне области $\Pi_{p, h}$. Тогда из (3.9) следует (3.8). Предложение доказано.

ПреДЛОЖЕНИЕ 3.2. Пусть Т - нормальный оператор с такой же локализаиией спектра, как в предложении 3.1. Пусть форма $\mathfrak{b}=\mathfrak{b}[x, x]$ является $p$-подчиненной оператору $T$ с точной гранъю $b^{\prime}$. Тогда спектр оператора $A=T+B$, расположенный внутри вдвое менъшего сектора $\Lambda_{\alpha / 2}$, асимптотически локализован в области $\Pi_{p, h}$, если $h>2 b^{\prime}$, а в области $\Lambda_{\alpha / 2} \backslash \Pi_{p, h}$ при любом $\varepsilon>0$ асимптотически выполняется оценка

$$
\left\|(A-\lambda)^{-1}\right\| \leqslant\left(\frac{2 b^{\prime}+\varepsilon}{h-2 b^{\prime}}\right) \frac{1}{|\tau|}, \quad \mid \lambda \gg 1 .
$$

ДокАЗАтЕльство. Будем проводить доказательство на основе представлений (2.12) и (2.13). В силу (3.6) получаем

$$
\begin{aligned}
&\left|\left(S_{1}(\lambda) x, x\right)\right|=\left|\left(S_{2}(\lambda) x, x\right)\right|=|(B V(\lambda) x, V(\lambda) x)| \\
& \leqslant b\left\|T^{1 / 2} V(\lambda) x\right\|^{2 p}\|V(\lambda) x\|^{2-2 p}+M\|V(\lambda) x\|^{2} \\
& \leqslant b\left\|T^{1 / 2}(T-\lambda)^{-1 / 2}\right\|^{2 p}\left\|(T-\lambda)^{-1 / 2}\right\|^{2-2 p}\|x\|^{2} \\
& \quad+M\left\|(T-\lambda)^{-1 / 2}\right\|^{2}\|x\|^{2} .
\end{aligned}
$$

Так же, как в предложении 3.1, из (2.1) получаем оценку

$$
\left\|T^{1 / 2}(T-\lambda)^{-1 / 2}\right\|^{2}=\left(\sup _{t \in \sigma(T)}\left|\frac{t}{t-\lambda}\right|^{1 / 2}\right)^{2} \leqslant \frac{|\lambda|}{|\tau|}, \quad\left\|(T-\lambda)^{-1 / 2}\right\|^{2} \leqslant \frac{1}{|\tau|} .
$$

Билинейная форма оператора $S_{1}$ в гильбертовом пространстве выражается через квадратичную, откуда следует, что $\left\|S_{1}\right\|=\left\|S_{2}\right\| \leqslant 2\left|\left(S_{1} x, x\right)\right|\|x\|^{-2}$. Завершается доказательство точно так же, как доказательство предложения 3.1, с той лишь разницей, что константы $b$ и $b^{\prime}$ удваиваются.

Мы отмечали, что условие $p$-подчиненности в смысле (3.1) сильнее, нежели условие (3.2). Однако его проще проверять и оно определено не только для 
$p \in[0,1)$, но и для всех отрицательных $p$. При $p<0$ спектр $T+B$ прижимается к вещественной оси и локализуется под гиперболами. Легко получается следующее предложение.

ПредЛОЖЕНИЕ 3.3. Пусть Т - нормальный оператор с такой же локализацией спектра, как в предложении 3.1. Если условие (3.1) выполнено при $p<0$, mo

$$
\left\|B(T-\lambda)^{-1}\right\| \leqslant C b \max \left(\frac{1}{|\lambda|}, \frac{|\lambda|^{p}}{\operatorname{dist}(\lambda, \sigma(T))}\right) .
$$

При этом спектр оператора $A=T+B$ локализуется в области следующего вида: $|\tau|<C_{1}|\lambda|^{p}$.

Другие формы $p$-подчиненных возмущений будут рассмотрены в разделе 7.

\section{4. Основная теорема об оценке резольвенты и теоремы о полноте}

В этом разделе мы предполагаем, что оператор $T$ плотно определен в пространстве $H$ и имеет компактную резольвенту. Не ограничивая общности, считаем, что $0 \in \rho(T)$, т. е. оператор $T^{-1}$ компактен. Тогда $T$ замкнут и множество $\mathscr{D}(T)$ после введения нормы графика

$$
\|x\|_{T}^{2}=\|T x\|^{2}+\|x\|^{2}
$$

превращается в гильбертово пространство, которое обозначим через $H_{T}$. Компактность оператора $T^{-1}$ влечет компактность вложения $H_{T} \hookrightarrow H$. Согласно теореме Дж. фон Неймана оператор $T^{*} T$ самосопряжен. Обозначим $S:=$ $\left(T^{*} T\right)^{1 / 2}$. Справедлива теорема о полярном разложении $T=U S$, где $U-$ унитарный оператор (здесь учитываем, что $T$ обратим).

Обозначим $K=T^{-1}$. Собственные значения положительного компактного оператора $\left(K K^{*}\right)^{1 / 2}$ называют s-числами оператора $K$. Из представления $T=U S$ получаем равенство $s_{j}\left(T^{-1}\right)=s_{j}^{-1}(S)$. Важно знать, что $s_{j}(K)$ имеют такой же порядок, как s-числа оператора вложения $I: H_{T} \rightarrow H$. Последняя задача хорошо изучена для функциональных пространств $H_{T}$ и $H$ (см., например, [59; гл. 4.10]). Отметим также, что вопросы об оценках и асимптотическом поведении s-чисел и собственных значений дифференциальных операторов изучены достаточно полно. Подробную информацию можно найти в обзорах [61], [62] и [63].

Далее, условимся говорить, что оператор $T$ имеет порядок $\alpha$, если

$$
s_{j}\left(T^{-1}\right) \leqslant c j^{-\alpha}, \quad c=\text { const } .
$$

Очевидно, что это условие эквивалентно следующему:

$$
\lambda_{j}(S) \geqslant c^{-1} j^{\alpha},
$$

где $\lambda_{j}(S)$ - собственные значения оператора $S$. Здесь собственные значения оператора $S$ нельзя заменить на собственные значения оператора $T$. Оператор $T$ может вовсе не иметь собственных значений (это так, если $T^{-1}$ - вольтерров оператор). Конечно, если $T$ - самосопряженный или нормальный оператор, 
то можно считать, что $S=|T|$. Тогда условие (4.1) эквивалентно следующему:

$$
\varlimsup_{t \rightarrow \infty} \frac{n(t)}{t^{\beta}} \leqslant c, \quad \beta=\alpha^{-1},
$$

где $n(t)=\sum_{\left|\mu_{k}\right|<t} 1-$ функция распределения собственных значений оператора T. Можно говорить о точном порядке оператора $T$. Он определяется как верхняя грань чисел $\alpha$ в неравенстве (4.1). Сделаем важное замечание: два оператора с дискретным спектром, имеющие одинаковую область определения, имеют одинаковые порядки. Действительно, если $S_{1}$ и $S_{2}$ - положительные операторы, осуществляющие биекцию $H_{T}$ на $H$, то оператор $S_{2} S_{1}^{-1}$ ограничен и обратим в $H$, а тогда из свойств s-чисел (см. [25; гл. 2]) следует, что $s_{j}\left(S_{1}^{-1}\right) \asymp s_{j}\left(S_{2}^{-1}\right)$.

Замечательную роль в теории несамосопряженных операторов играют приведенные ниже теоремы об оценках резольвенты. Первая из них дает оценку фредгольмовой резольвенты ядерного оператора, а вторая (основная) - представление для резольвенты неограниченного оператора конечного порядка $\alpha$.

Напомним, что оператор $K$ называется ядерным, если $\sum s_{j}(K)<\infty$. Оператор-функция $(1-\lambda K)^{-1}$ называется фредгольмовой резольвентой.

Теорема 4.1. Пусть $\left\{s_{j}\right\}_{1}^{\infty} u\left\{\lambda_{j}\right\}_{1}^{\infty}$-s-числа и собственные значения (занумерованные с учетом алгебраической кратности) ядерного оператора $K$. Тогда

$$
(1-\lambda K)^{-1}=\frac{F(\lambda)}{D(\lambda)}, \quad \text { где } \quad D(\lambda)=\prod_{j=1}^{\infty}\left(1-\lambda \lambda_{j}\right) .
$$

Произведение $D(\lambda)$ сходится и является целой функиией порядка $\leqslant 1$ и минимального типа при порядке 1. Оператор-функиия $F(\lambda)$ также иелая и допускает оченку

$$
\|F(\lambda)\| \leqslant \prod_{j=1}^{\infty}\left(1+|\lambda| s_{j}\right) .
$$

Тем самым, $F(\lambda)$ также имеет порядок $\leqslant 1$ и минимальный тип при порядке 1 .

По существу эта теорема принадлежит М. В. Келдышу [1], [3]. В своем доказательстве он опирался на более ранний результат Т. Карлемана [5], который формулируется так: если оператор $K^{2}$ ядерный и вольтерров (т.е. не имеет собственных значений), то фредгольмова резольвента оператора $K$ есть целая функиия порядка роста 2 и минимального типа.

После выхода работы [1], в которой результаты были только анонсированы, независимые доказательства некоторых вариантов теоремы 4.1 получили Ф. Браудер [6], М. Г. Крейн [12], С. Агмон [21] и В. Б. Лидский [14]. Во всех этих работах для доказательства привлекались сложные в техническом отношении результаты об оценках характеристических определителей бесконечного порядка. Неожиданно короткое и элегантное доказательство этой теоремы нашел В. И. Мацаев [20]. Это доказательство с некоторой модификацией приведено в книге И. Ц. Гохберга и М. Г. Крейна [25; гл. 5, теорема 5.1]. 
Теорема 4.2. Пусть $T$ - неограниченный оператор конечного порядка $\alpha$. Тогда его резольвента является мероморфной оператор-функиией

$$
(T-\lambda)^{-1}=\frac{\Phi(\lambda)}{D(\lambda)}
$$

где $D$ - скалярная функиия, а $\Phi$ - оператор-функиия со значениями в идеале компактных операторов. Обе функции - целье порядка роста $\leqslant \alpha^{-1} u$ нормального типа при порядке $\alpha^{-1}$. Фредголвмова резолввента $T(T-\lambda)^{-1}=$ $\left(1-\lambda T^{-1}\right)^{-1}$, как оператор-функция со значениями в пространстве ограниченных операторов, обладает тем же свойством.

Заметим, что в литературе можно встретить другие (эквивалентные и не эквивалентные) формулировки этой важной теоремы. Мы отдали предпочтение приведенной формулировке, считая ее наиболее удобной для приложений. Мы не знаем работы, где можно было бы найти короткое доказательство сформулированной теоремы (из результатов работы [3] для фредгольмовой резольвенты оператора $T^{-1}$ получается менее точный результат). Поэтому мы приведем здесь полное доказательство.

ДоКАЗАТЕЛЬСтво теоремы 4.2. Через $\left\{\mu_{j}\right\}$ обозначаем собственные значения оператора $T$, а через $\left\{\lambda_{j}\right\}=\left\{\mu_{j}^{-1}\right\}$ и $\left\{s_{j}\right\}$ - собственные значения и s-числа оператора $K=T^{-1}$. Считаем, что числа $\left\{\lambda_{j}\right\}$ и $\left\{s_{j}\right\}$ занумерованы в порядке убывания модулей. Сначала рассмотрим случай, когда порядок $T$ больше 1. Согласно определению, из (4.1) получаем, что в этом случае оператор $K$ ядерный, а тогда сходятся канонические произведения

$$
D(\lambda):=\prod_{j=1}^{\infty}\left(1-\lambda \lambda_{j}\right)=\prod_{j=1}^{\infty}\left(1-\frac{\lambda}{\mu_{j}}\right), \quad S(\lambda):=\prod_{j=1}^{\infty}\left(1+\lambda s_{j}\right) .
$$

При этом сходимость первого произведения обеспечивается оценкой

$$
\prod_{j=1}^{n}\left(1+|\lambda|\left|\lambda_{j}\right|\right) \leqslant \prod_{j=1}^{n}\left(1+|\lambda| s_{j}\right)
$$

которая верна при любом $n \in \mathbb{N}$. Эта оценка есть следствие теоремы Вейля [64] о свойствах s-чисел компактных операторов (см. [64], [25; гл. 2, следствие 3.1]).

Пусть $s(t)=\sum_{s_{k} \geqslant 1 / t} 1-$ функция распределения нулей функции $S(\lambda)$, определенной в (4.4). Очевидно, что условие (4.1) влечет оценку

$$
s(t) \leqslant c t^{\beta}, \quad \beta=\alpha^{-1}
$$

(верно и обратное утверждение). 
Известно [65; теорема 4.2], что

$$
\ln |S(\lambda)| \leqslant C\left(\int_{0}^{r} \frac{s(t)}{t} d t+r \int_{r}^{\infty} \frac{s(t)}{t} d t\right), \quad r=|\lambda|, \quad C=\text { const } .
$$

Тогда из (4.6) следует неравенство $\ln |S(\lambda)| \leqslant r^{\beta}$, т. е. функция $S(\lambda)$ имеет порядок роста $\leqslant \beta$ и нормальный тип при порядке $\beta$. Из (4.5) получаем, что тем же свойством обладает функция $D(\lambda)$.

Согласно теореме 4.1 фредгольмова резольвента $T(T-\lambda)^{-1}$ представима как отношение функций $F$ и $D$, при этом $\|F(\lambda)\| \leqslant S(|\lambda|)$. Так как порядки функций $S$ и $D$ не превышают $\beta$, то мероморфная функция $\left(1-\lambda T^{-1}\right)^{-1}$ (а тогда и $\left.(T-\lambda)^{-1}\right)$ имеет порядок $\leqslant \beta$ и нормальный тип.

При $\alpha \leqslant 1$ выберем число $q \in \mathbb{N}$ такое, что $\alpha q>1$, и воспользуемся равенствами

$$
\begin{aligned}
\left(1-\lambda^{q} K^{q}\right) & =(1-\lambda K)\left(1+\lambda K+\cdots+\lambda^{q-1} K^{q-1}\right), \\
(1-\lambda K)^{-1} & =\left(1+\lambda K+\cdots+\lambda^{q-1} K^{q-1}\right)\left(1-\lambda^{q} K^{q}\right)^{-1} .
\end{aligned}
$$

Из неравенств $s_{j}\left(K^{q}\right) \leqslant s_{j}^{q}(K)$ [25; гл. 2] следует, что оператор $K^{q}$ ядерный, а его фредгольмова резольвента $\left(1-\zeta K^{q}\right)^{-1}$ является мероморфной оператор-функцией порядка роста $\leqslant(\alpha q)^{-1}$ и нормального типа при порядке $(\alpha q)^{-1}$. Следовательно, оператор-функция $\left(1-\lambda^{q} K^{q}\right)^{-1}$ является мероморфной функцией и имеет порядок роста $\leqslant \alpha^{-1}$ и нормальный тип при порядке $\leqslant \alpha^{-1}$. Из равенства (4.7) получаем, что этим же свойством обладает оператор-функция $(1-\lambda K)^{-1}$, а тогда и $T^{-1}(1-\lambda K)^{-1}$. Этим завершается доказательство теоремы.

ЗАмЕчАниЕ 4.3. Из приведенного доказательства следует, что постоянная $c$ в неравенствах (4.1) отвечает за тип мероморфной функции $(T-\lambda)^{-1}$. В частности, если в (4.1) постоянную $с$ можно взять произвольно большой, то $(T-\lambda)^{-1}$ будет функцией минимального типа при порядке $\alpha^{-1}$. Легко также видеть, что если $T^{-1}$ принадлежит при некотором $\beta>0$ идеалу Неймана-Шаттена $\mathfrak{S}_{\beta}[25$; гл. 3], т. е. если

$$
\sum s_{j}^{\beta}\left(T^{-1}\right)<\infty
$$

то резольвента $(T-\lambda)^{-1}$ является мероморфной оператор-функцией порядка $\beta$ и минимального типа. Этот факт доказывается с помощью тех же аргументов, что и выше.

Следующая теорема дает представление резольвенты оператора в малой окрестности полюса.

Теорема 4.4. Главная часть резольвенты $(T-\lambda)^{-1}$ в окрестности полюса $\lambda=\mu$ имеет представление

$$
\sum_{j=1}^{N} \sum_{s=0}^{p_{j}} \frac{\left(\cdot, \psi_{j}^{0}\right) \varphi_{j}^{0}+\left(\cdot, \psi_{j}^{1}\right) \varphi_{j}^{1}+\cdots+\left(\cdot, \psi_{j}^{s}\right) \varphi_{j}^{s}}{(\lambda-\mu)^{p_{j}+1-s}}
$$

¿əe

$$
\varphi_{j}^{0}, \varphi_{j}^{1}, \ldots, \varphi_{j}^{p_{j}}, \quad j=1, \ldots, N
$$


- каноническая система жордановых цепочек (корневых векторов) оператора $T$, отвечающая собственному значению $\mu, a$

$$
\psi_{j}^{0}, \psi_{j}^{1}, \ldots, \psi_{j}^{p_{j}}, \quad j=1, \ldots, N,
$$

- однозначно определяемая по системе (4.8) каноническая система жордановых чепочек оператора $T^{*}$.

ДокАзАТЕльство. В работе [3] эта теорема доказана для мероморфных оператор-функций $L^{-1}(\lambda)$ в случае, когда $L(\lambda)$ - полином с операторными коэффициентами (достаточно требовать аналитичности $L(\lambda)$ в окрестности нормального собственного значения $\mu$ ). Доказательство основано на следующем простом утверждении: соотношение

$$
L(\lambda)\left[\varphi^{0}+(\lambda-\mu) \varphi^{1}+\cdots+(\lambda-\mu)^{s} \varphi^{s}\right]=O(\lambda-\mu)^{s+1} \quad \text { при } \lambda \rightarrow \mu
$$

имеет место в том и только том случае, когда $\varphi^{0}, \ldots, \varphi^{s}-$ жорданова цепочка оператор-функции $L(\lambda)$, отвечающая собственному значению $\mu$.

Теперь мы готовы дать простое доказательство теорем о полноте.

ОПРЕДЕЛЕНиЕ 4.5. Будем говорить, что оператор $T$ принадлежит классу $\mathscr{K}_{\alpha}, 0<\alpha \leqslant 2$, если найдутся лучи $\gamma_{1}, \ldots, \gamma_{N}$ наилучшего убывания его резольвенты такие, что все углы между соседними лучами не больше $\pi \alpha$.

ЗАмЕчАНИЕ 4.6. Из этого определения и замечания 2.4 следует, что если $T \in \mathscr{K}_{\alpha}$, то $T \in \mathscr{K}_{\alpha-\varepsilon}$ при некотором $\varepsilon>0$.

В частности, если $T$ имеет хотя бы один луч наилучшего убывания резольвенты, то $T \in \mathscr{K}_{2}$. Если $T-m$-секториальный оператор с углом $2 \pi \beta<\pi$ (т. е. значения квадратичной формы $(T x, x)$ лежат в секторе $|\arg \lambda| \leqslant \pi \beta$ и внешность этого сектора принадлежит резольвентному множеству $\rho(T))$, то $T \in$ $\mathscr{K}_{2 \beta+\varepsilon}$ при любом $\varepsilon>0$. Любой самосопряженный оператор, а также любой нормальный оператор со спектром, локализованным на конечном числе лучей, принадлежит классу $\mathscr{K}_{\varepsilon}$ при любом $\varepsilon>0$.

Напомним, что система векторов $\left\{\varphi_{k}\right\}$ называется полной в $H$, если в $H$ нет ненулевых векторов, ортогональных всем векторам системы. Эквивалентное определение такое: любой вектор $x \in H$ можно с любой точностью приблизить линейными комбинациями векторов из системы.

Теорема 4.7. Пусть оператор $T$ имеет порядок $\alpha$ и принадлежит классу $\mathscr{K}_{\gamma}$ при некотором $\gamma \leqslant \alpha$. Тогда его система корневых векторов полна в пространстве $H$.

ДокАЗАТЕЛЬство. Предположим, что существует вектор $x \in H$, ортогональный всем корневым векторам оператора $T$. Тогда согласно теоремам 4.2 и 4.4 скалярная функция

$$
F_{y}(\lambda)=\left((T-\lambda)^{-1} y, x\right)
$$

является целой порядка роста $\leqslant \alpha^{-1}$. Из условия $T \in \mathscr{K}_{\gamma}$ следует, что эта функция убывает на лучах $\gamma_{1}, \ldots, \gamma_{N}$, раствор между которыми не больше $\pi \gamma$. В силу замечания 4.6 можно считать, что раствор между этими лучами строго меньше $\pi \gamma$, а потому и строго меньше $\pi \alpha$. Согласно теореме Фрагмена-Линделёфа 
(см., например, $[65]), F_{y}(\lambda)$ убывает при $\lambda \rightarrow \infty$ внутри каждого из секторов между соседними лучами, а тогда по теореме Лиувилля $F_{y}(\lambda) \equiv 0$. Но из равенства $F_{y}(\lambda)$ при всех $y \in H$ получаем $x=0$. Теорема доказана.

ЗАмЕчАниЕ 4.8. Условия теоремы 4.7 можно ослабить, а именно, достаточно требовать, чтобы резольвента $(T-\lambda)^{-1}$ на лучах $\gamma_{k}$ росла не быстрее некоторой степени $\lambda$. Но в этом случае условие $\gamma \leqslant \alpha$ должно быть заменено на условие $\gamma<\alpha$. Доказательство остается почти таким же. Вместо скалярной функции $F_{y}$ рассматриваем вектор-функцию $F(\lambda)=\left[(T-\lambda)^{-1}\right]^{*} x$, предполагая, что $x$ ортогонален всем корневым векторам оператора $T$. Тогда функция $F$ целая порядка роста $\leqslant \alpha^{-1}$. Из теоремы Фрагмена-Линделёфа заключаем, что $F$ растет не быстрее полинома при $\lambda \rightarrow \infty$, поэтому

$$
F(\lambda)=y_{0}+y_{1} \lambda+\cdots+y_{p} \lambda^{p}, \quad \text { где } y_{j} \in \mathscr{D}\left(T^{*}\right) .
$$

Но тогда $x=\left(T^{*}-\lambda\right)\left(y_{0}+y_{1} \lambda+\cdots+y_{p} \lambda^{p}\right)=0$. Сравнивая коэффициенты при степенях $\lambda$, получаем $y_{p}=y_{p-1}=y_{0}$, а тогда $x=0$.

СлеДСтвиЕ 4.9. Пусть оператор $T$ имеет порядок $\alpha$ u $T \in \mathscr{K}_{\gamma}$ при некотором $\gamma \leqslant \alpha$. Если оператор $B$ вполне T-подчинен, то система корневых векторов оператора $T+B$ полна в пространстве $H$. Это утверждение сохраняет силу, если $T$ - нормальный оператор, а $B$ порождается вполне T-подчиненной квадратичной формой.

ДокАЗАТЕЛЬСтво. Достаточно воспользоваться теоремой 2.5.

СледСтвиЕ 4.10. Пусть T-m-секториальный оператор, причем значения его квадратичной формы лежат в угле $c|\arg \lambda| \leqslant \gamma \pi / 2$. Пусть $T$ имеет конечный порядок $\alpha$, причем $\gamma<\alpha$. Если оператор $B$ вполне $T$-подчинен, то система корневых векторов оператора $T+B$ полна в пространстве $H$.

СлеДСТВИЕ 4.11. Пусть Т - самосопряженный оператор любого конечного порядка или нормальныи оператор со спектром на конечном числе лучей. Если оператор $B$ вполне T-подчинен или порождается вполне T-подчиненной квадратичной формой, то система корневых векторов оператора $T+B$ полна в пространстве $H$.

ДокАзАТЕЛЬство. Достаточно заметить, что оператор $T$ (а тогда и оператор $T+B)$ принадлежит классу $\mathscr{K}_{\varepsilon}$ при любом $\varepsilon>0$.

Отметим, что первое утверждение следствия 4.11 (в случае, когда $B$ вполне $T$-подчинен) представляет собой эквивалентную форму одной из основных теорем Келдыша о полноте.

Может возникнуть вопрос: почему теоремы о полноте мы формулируем в терминах принадлежности операторов классу $\mathscr{K}_{\alpha}$ ? Ответ на этот вопрос простой. Во-первых, такая формулировка позволяет получить теоремы о полноте для возмущений самосопряженных и нормальных операторов как простое следствие полученных ранее оценок для резольвенты возмущенного оператора $T+B$. А во-вторых (и это главное), задача об оценках резольвенты общих классов дифференциальных операторов (не обязательно самосопряженных или нормальных) хорошо изучена. Для обыкновенных дифференциальных 
операторов, порожденных регулярными краевыми условиями, еще Дж. Биркгоф [66], [67] показал, что все лучи комплексной плоскости, кроме $\mathbb{R}_{+}$и $\mathbb{R}_{-}$, являются лучами наилучшего убывания резольвенты, а для операторов с частными производными, порожденных регулярными по Лопатинскому краевыми условиями, нужные оценки для резольвенты в секторах комплексной плоскости получили С. Агмон [21] и М. С. Агранович и М.И. Вишик [68]. Более современное изложение этих вопросов можно найти, например, в монографиях M. А. Наймарка (для обыкновенных дифференциальных операторов) [69; гл. 1] и Х. Трибеля [59; гл. 5].

\section{5. Суммируемость спектральных разложений методом Абеля-Лидского}

Условия, при которых получены теоремы о полноте, не гарантируют сходимости спектральных разложений

$$
\sum_{k=1}^{n} P_{k} x \rightarrow x \quad \text { при } n \rightarrow \infty,
$$

где $P_{k}=-\frac{1}{2 \pi i} \int_{\Gamma_{k}}(T-\lambda)^{-1} d \lambda$ - проекторы Рисса на корневые подпространства оператора $T$. Здесь $\Gamma_{k}$ - положительно ориентированные замкнутые контуры, заключающие внутри себя одно или несколько собственных значений оператора $T$. Интересен ответ на вопрос о возможности суммирования этих разложений. Предположим, что спектр оператора $T$ лежит в секторе

$$
\Lambda_{\tau}=\left\{\lambda \in \mathbb{C}:|\arg \lambda| \leqslant \frac{\tau \pi}{2}\right\}, \quad 0 \leqslant \tau<2 .
$$

Функция $\exp \left(-\lambda^{\beta} t\right)$ при любом $t>0$ экспоненциально убывает в этом секторе, если только $\beta \tau<1$. В этом случае $\varepsilon=\cos (\beta \tau \pi / 2)>0$ и указанная функция оценивается сверху величиной $\exp \left(-\varepsilon|\lambda|^{\beta} t\right)$. Образуем ряд

$$
S(t)=\sum_{k=1}^{\infty} S_{k}(t), \quad \text { где } \quad S_{k}(t)=-\frac{1}{2 \pi i} \int_{\Gamma_{k}} e^{-\lambda^{\beta} t}(T-\lambda)^{-1} d \lambda .
$$

Введение экспоненциально убывающего множителя может обеспечить сходимость этого ряда. Если при любом $t>0$ сильная сходимость ряда имеет место и, кроме того,

$$
\lim _{t \rightarrow 0+} S(t) x=x \quad \forall x \in H,
$$

то будем говорить, что система корневых векторов оператора $T$ образует базис для метода суммирования Абеля-Лидского порядка $\beta$.

Вопрос о суммируемости спектральных разложений в указанном смысле впервые исследовал В. Б. Лидский [14], [15]. В работе [14] он доказал утверждение о возможности суммирования разложений указанным методом порядка $\beta$ для $m$-секториальных операторов с углом $\Lambda_{\tau}$ при условии $\beta \in\left(\alpha^{-1}, \tau^{-1}\right), \tau<\alpha$, где $\alpha$ - порядок оператора. Однако сходимость была им доказана не при всех 
$x \in H$, а только при $x \in \mathscr{D}(T)$. Это ограничение впоследствии снял В. И. Мацаев. В работе [15] В. Б. Лидский заметил, что порядок суммирования $\beta$ можно понижать, допуская значения $\beta<\alpha^{-1}$, если задачу о суммировании рассматривать для оператора $A=T+B$, где $B$ является сильно $p$-подчиненным оператору $T=T^{*}>0, p<1$. Однако это замечание было им сделано не для общего случая, а для случая самосопряженного эллиптического оператора второго порядка, возмущенного несамосопряженным оператором первого порядка. В общем случае возможность понижения порядка суммируемости до числа $\beta>\alpha-1+p$ для сильно $p$-подчиненных возмущений доказал В.Э. Кацнельсон [23]. Изложение этих вопросов (но без подробных доказательств) имеется в [33], а для $p$-подчиненных возмущений в смысле форм (с доказательствами) - в [60].

В [70]-[72] замечено, что задачу о суммировании методом Абеля-Лидского можно эффективно решать не средствами спектральной теории, а средствами теории функций. Используя результаты работы [70] об оценках мероморфных функций, можно получить более общие результаты и при этом явно указать правило расстановки скобок при суммировании, т. е. правило выбора контуров $\Gamma_{k}$ в (5.2). Здесь мы изложим вкратце суть вопроса.

Для доказательства нижеследующей теоремы 5.1 Лидского-Мацаева будем использовать теорему Тичмарша (см., например, [65]): если иелая функиия $\Delta(\lambda)$ имеет порядок роста $\leqslant \nu$, то для любого $\varepsilon>0$ найдется последовательность чисел $r_{j} \rightarrow \infty$ таких, что на окружностях $|\lambda|=r_{j}$ эта функиия допускает оценку снизу

$$
|\Delta(\lambda)| \geqslant C \exp \left(-|\lambda|^{\nu+\varepsilon}\right)
$$

Tеорема 5.1. Пусть оператор T имеет порядок $\alpha$ и его спектр, за исключением конечного числа собственных значений, лежит в секторе $\Lambda_{\tau}$, определенном формулой (5.1), причем $\alpha>\tau$. Пусть все лучи вне сектора $\Lambda_{\tau}$ являются лучами наилучшего убывания резольвенты оператора T. Тогда система корневых векторов оператора Т образует базис для метода суммирования Абеля-Лидского порядка $\beta$ при любом $\beta \in\left(\alpha^{-1}, \tau^{-1}\right)$.

ДокАзАтЕЛЬство. Обозначим через Г ориентированный контур, являющийся границей области, состоящей из объединения сектора $\Lambda_{\tau+\delta}$ и круга достаточно большого радиуса (такого, что вне этой области нет собственных значений оператора $T)$. Число $\delta>0$ возьмем столь малым, что $\beta<(\tau+\delta)^{-1}$. Рассмотрим оператор

$$
S(t)=-\frac{1}{2 \pi i} \int_{\Gamma} e^{-\lambda^{\beta} t}(T-\lambda)^{-1} d \lambda
$$

где выбрана главная ветвь корня функции $\lambda^{\beta}$. Заметим, что оператор $S(t)$ корректно определен. Это следует из экспоненциального убывания подынтегральной функции, что гарантируется выбором числа $\delta$. Так как $\beta>\alpha^{-1}$, то из теоремы 4.2 и теоремы Тичмарша следует, что при любом $t>0$ подынтегральная функция экспоненциально убывает на некоторой последовательности уходящих на бесконечность дуг $\gamma_{k}:|\lambda|=r_{k}$. Обозначим через $\Gamma_{k}$ ориентированные контуры, которые являются границами областей, заключенных в секторе $\Lambda_{\tau+\delta}$ 
между дугами $\gamma_{k}$ и $\gamma_{k+1}$. В силу экспоненциального убывания подынтегральной функции на контурах $\Gamma_{k}$ оператор $S(t)$ при любом $t>0$ представляется сходящимся по операторной норме рядом проекторов $S_{j}(t)$ на корневые подпространства оператора $T$, которые отвечают за собственные значения, заключенные между дугами $\gamma_{k}$ и $\gamma_{k+1}$. Следовательно, $S(t)$ совпадает с рядом (5.2), причем выбор контуров $\Gamma_{k}$ (или, что эквивалентно, выбор расстановки скобок при суммировании) не зависит от $x$ и $t$, а зависит только от расположения собственных значений $\mu_{k}$ оператора $T$.

Остается доказать соотношение (5.2). Заметим, что оно заведомо выполняется, если вектор $x$ есть конечная линейная комбинация корневых векторов оператора $T$ (в этом случае число членов ряда конечно). Но согласно теореме 4.7 (ее условия выполнены) имеется полнота корневых векторов, поэтому соотношение (5.2) выполняется на плотном множестве в $H$. Тогда остается только показать, что нормы $\|S(t)\|$ равномерно ограничены при $t \rightarrow 0+$. Именно этот факт был замечен Мацаевым (доказательство имеется в [33], но для удобства читателя мы воспроизведем его здесь).

Не ограничивая общности, считаем, что оператор $T$ обратим. Замена конечного участка контура Г другим не влияет на равномерную оценку интеграла $S(t)$ при $t>0$. Заменим контур $\Gamma$ на $\Gamma^{\prime}-$ границу сектора $\Lambda_{\tau+\delta}$ (при этом $\delta$ надо взять так, чтобы собственные значения $T$ не попадали на $\Gamma^{\prime}$ ). Сделаем замену $\zeta=\lambda t^{1 / \beta}$. Тогда контур $\Gamma^{\prime}$ перейдет в себя и мы получим

$$
S(t)=\frac{-t^{-1 / \beta}}{2 \pi i} \int_{\Gamma} e^{-\zeta^{\beta}}\left(T-\zeta t^{-1 / \beta}\right)^{-1} d \zeta .
$$

Интегралы по частям контура $\Gamma^{\prime}$ при $|\zeta| \geqslant 1$ легко оцениваются, поскольку подынтегральная функция оценивается величиной $\exp \left(-\varepsilon|\zeta|^{\beta}\right)|\zeta|^{-1} t^{1 / \beta}$. Интегралы по $\Gamma^{\prime}$ при $|\zeta| \leqslant 1$ можно заменить интегралом по части окружности $|\zeta|=1$ вне сектора $\Lambda_{\tau+\delta}$ и двух берегов отрезка $[-1,0]$. Интеграл по дуге окружности оценивается с помощью оценки $\left\|(T-\lambda)^{-1}\right\| \leqslant C|\lambda|^{-1}$ вне сектора $\Lambda_{\tau+\delta}$, а оценка суммы интегралов по берегам отрезка $[-1,0]$ сводится к оценке интеграла

$$
\int_{-1}^{0} e^{-\varepsilon|\zeta|^{\beta}} \sin \left(\varepsilon|\zeta|^{\beta}\right)|\zeta|^{-1} d \zeta,
$$

который, очевидно, сходится. Теорема доказана.

Доказательство приводимой далее теоремы 5.3 о возможности понижения порядка суммируемости и указания правила расстановки скобок основано на следующей теореме автора [72; теоремы 5.2 и 5.3], которую мы формулируем здесь в упрощенном виде.

ТЕОРема 5.2. Пусть $f$-мероморфная функиия конечного порядка в некотором секторе $\Lambda_{\tau}$, причем ее полюсы лежат в параболической области

$$
\Pi_{p, h}=\left\{\lambda=\sigma+i \eta \in \mathbb{C}:|\eta| \leqslant h \sigma^{p}, \sigma>0\right\}, \quad p<1,
$$

а при некотором $\varepsilon>0$ на границе области $\Pi_{p, h+\varepsilon}$ верна оценка $|f(\lambda)| \leqslant C$. Тогда найдется последовательность $r_{1}<r_{2}<\cdots<r_{k} \rightarrow \infty$ такая, что при 
$\lambda \in \Pi_{p, h+\varepsilon},|\lambda|=r_{k}$, выполняется оченка

$$
\ln |f(\lambda)| \leqslant C_{1}\left(1+r^{p-1} n(r)\right), \quad r=|\lambda|,
$$

где $n(r)$ - функиия распределения полюсов $f$, расположенных в области $\Pi_{p, h}$, а постоянная $C_{1}$ зависит только от $C$ и расположения полюсов $f$, но не зави-

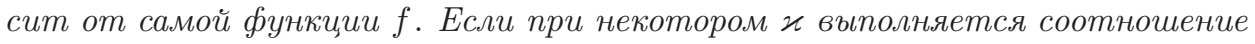

$$
n\left(r+r^{p}\right)-n(r)=O\left(r^{\varkappa}\right)
$$

mo

$$
\ln |f(\lambda)|=O\left(r^{\varkappa}\right)
$$

для всех $\lambda \in \Pi_{p, h+\varepsilon}$, но вне некоторого исключительного множества кружков $\mathscr{K}$. В качестве исключительного множества при любом $\omega>\varkappa$ можно взять

$$
\mathscr{K}=\bigcup_{j} \mathscr{K}_{j}, \quad \text { əде } \quad \mathscr{K}_{j}=\left\{\lambda:\left|\lambda-\mu_{j}\right| \leqslant \exp \left(-\left|\mu_{j}\right|^{\omega-\varkappa}\right)\right\} .
$$

Из этой теоремы сразу получаем усиление теоремы Кацнельсона [23] и теорему Аграновича [60].

ТЕОРема 5.3. Пусть $T$ - самосопряженный полуограниченный снизу оператор порядка $\alpha$, а оператор $B$ является р-подчиненным оператору $T$ или порождается р-подчиненной оператору $T$ квадратичной формой. Тогда система корневых векторов оператора $A=T+B$ образует базис для метода суммирования Абеля порядка $\beta$ при любом $\beta>\alpha^{-1}-1+p$.

ДокАЗАтЕльство. В силу предложений 3.1 и 3.2 спектр возмущенного оператора $A=T+B$ лежит в объединении круга достаточно большого радиуса и параболической области $\Pi_{p, h}$ при некотором $h>0$. Вне этого круга и области $\Pi_{p, h}$ спектра нет, а резольвента $(A-\lambda)^{-1}$ ограничена. Согласно теореме 2.5 вне произвольно малого сектора $\Lambda_{\varepsilon}$ резольвента имеет наилучшее убывание.

Очевидно, что порядки операторов $T$ и $A$ одинаковы. Это следует из определения, если $\mathscr{D}(T)=\mathscr{D}(A)$. В случае, когда $T$ возмущается $p$-подчиненной формой, имеем $T=R U R, A=R V R$, где $R=|T|^{1 / 2}, U$ - унитарный, а $V$ ограничен и обратим в $H$ (считаем, что $0 \notin \sigma(T) \cup \sigma(A))$. Но тогда $s_{j}\left(T^{-1}\right) \asymp s_{j}\left(A^{-1}\right)$, что следует из свойств s-чисел операторов. Следовательно, скалярная функция $D(\lambda)$, являющаяся знаменателем в представлении резольвенты $(A-\lambda)^{-1}$ из теоремы 4.2, имеет порядок $\alpha^{-1}$ и нормальный тип при этом порядке. Из теоремы Йенсена (см., например, [65]) следует, что для $n(t)$ - функции распределения нулей $D(\lambda)$ имеет место оценка

$$
\varlimsup_{r \rightarrow \infty} n(r) r^{-\beta}<C, \quad \beta=\alpha^{-1} .
$$

Рассмотрим скалярную функцию

$$
f(\lambda)=\left((A-\lambda)^{-1} x, y\right), \quad x, y \in H, \quad\|x\|=\|y\|=1 .
$$

В силу теоремы $5.2|f(\lambda)| \leqslant \exp \left(r^{\beta+p-1}\right)$ внутри $\Pi_{p, h}$ на последовательности дуг, уходящих в бесконечность, причем константа $C$ в этой оценке не зависит 
от $x, y$. Следовательно, $\ln \left\|(A-\lambda)^{-1}\right\|$ допускает такую же оценку. Поэтому при $\beta>\alpha^{-1}+p-1$ метод Абеля корректно определен: ряд проекторов $S_{k}(t)$ в (5.2), определенных для оператора $A$, сходится при всех $t>0$. Сходимость сумм $S(t) x \rightarrow x$ при $t \rightarrow 0+$ доказывается с помощью приема Мацаева точно так же, как в теореме 5.1.

Добавим только, что если выполнено условие (5.4), то при $\beta>\varkappa$ правило расстановки скобок можно указать эффективно: в скобки нужно объединять только члены, которые отвечают экспоненциально близким собственным значениям, как это указано в теореме 5.3. Теорема доказана.

ЗАмечАниЕ 5.4. Теорему 5.3 можно сформулировать в более общем виде, не требуя, чтобы оператор $A$ был возмущением положительного оператора $T$. Достаточно только следующее: спектр оператора $A$ локализован в параболе, вне параболы резольвента $(A-\lambda)^{-1}$ ограничена, а вне произвольно малого сектора $\Lambda_{\varepsilon}$ резольвента имеет наилучшее убывание.

Приведенное здесь доказательство теоремы 5.3 является коротким по форме, но по сути таковым не является. Доказательство теоремы 5.2, на которую мы опирались, требует нелегкой работы. Эта работа даже труднее, нежели прямое использование спектральных методов, речь о которых пойдет ниже. Но у предложенного здесь функционального подхода есть важное преимущество: если имеется информация о регулярности роста функции распределения полюсов в виде оценки (5.4), то эффективно указывается правило расстановки скобок.

\section{6. Теоремы о безусловной базисности}

Напомним известные понятия базиса и безусловного базиса (см., например, [25; гл. 6]). Последовательность векторов $\left\{\psi_{k}\right\}_{k=1}^{\infty}$ пространства $H$ называется базисом этого пространства, если каждый вектор $f \in H$ разлагается единственным образом в ряд

$$
f=\sum c_{k} \psi_{k}
$$

сходящийся по норме $H$.

Базис называется безусловным, если он остается базисом после любой перестановки векторов последовательности. Система $\left\{\psi_{k}\right\}_{k=1}^{\infty}$ называется базисом со скобками, если для любого $f \in H$ ряд (6.1) сходится по норме $H$ после некоторой расстановки скобок, не зависящей от $f$. Если система остается базисом после любой перестановки наборов ее векторов, которые отвечают членам ряда, заключаемым в скобки, то такая система называется безусловным базисом со скобками или базисом Рисса со скобками.

Систему $\left\{\psi_{k}\right\}_{k=1}^{\infty}$ называют минималъной, если существует система $\left\{\psi_{k}^{*}\right\}_{k=1}^{\infty}$ такая, что $\left(\psi_{k}, \psi_{j}^{*}\right)=\delta_{k j}$. Такую систему называют сопряженной или биортогональной; она определяется однозначно, если исходная система полна. Ряд (6.1) после заключения его членов в скобки можно переписать в виде

$$
x=\sum_{j=1}^{\infty} Q_{k} x, \quad Q_{j}=\sum_{k=r_{j}}^{r_{j+1}}\left(\cdot, \psi_{k}^{*}\right) \psi_{k},
$$


где $1=r_{1}, r_{2}, \ldots$ - возрастающая последовательность натуральных чисел, отвечающая за расстановку скобок, а $\left\{\psi_{k}^{*}\right\}_{k=1}^{\infty}-$ сопряженная система. Операторы $Q_{j}$ являются проекторами, обладающими свойством $Q_{j} Q_{k}=\delta_{k j}$.

Далее о базисных свойствах систем удобно говорить на языке проекторов. При этом естественно использовать следующие определения:

(а) система проекторов $\left\{Q_{j}\right\}_{1}^{\infty}$ полна, если равенства $\left(Q_{j} x, y\right)=0 \forall x \in H$, $j=1,2, \ldots$, влекут за собой равенство $y=0$;

(b) система $\left\{Q_{j}\right\}_{1}^{\infty}$ минималъна, если $Q_{j} Q_{k}=\delta_{k j}$.

Очевидно, что свойства полноты и минимальности являются необходимыми условиями базисности. Поэтому задача о свойствах базисности (безусловной базисности) систем в терминах проекторов получает такую формулировку. Дана полная и минимальная система проекторов $\left\{Q_{j}\right\}_{1}^{\infty}$. Найти условия, при которых частичные суммы

$$
S_{n}=\sum_{j=1}^{n} Q_{j}
$$

сходятся (безусловно сходятся) в сильном смысле к единичному оператору. При этом достаточно доказывать только сходимость, не требуя, чтобы предельный оператор был тождественным. Действительно, если сходимость частичных сумм $S_{n}$ имеет место, то по теореме Банаха-Штейнгауза $\sup \left\|S_{n}\right\|<\infty$. Но на подпространствах $S_{n}(H)$, образующих плотное множество в $H$, выполнено равенство $S_{n} x=x$, поэтому предельный оператор обязан быть единичным. В частности, формулировка задачи о базисности в терминах проекторов позволяет получить следующее утверждение: если система является базисом (безусловным базисом) со скобками, то сопряженная к ней система обладает тем же свойством.

При изучении проблемы базисности полезен следующий результат.

Теорема 6.1. Пусть $Q_{j}$ - полная и минимальная система проекторов. Следуюшие условия эквивалентны:

(i) ряд $\sum_{j=1}^{\infty} Q_{j}$ сходится безусловно;

(ii) имеет место оценка

$$
\sum_{j=1}^{\infty}\left|\left(Q_{k} x, x\right)\right|<\infty \quad \forall x \in H ;
$$

(iii) существуют полная и минимальная система ортогональньх проекторов $\left\{P_{j}\right\}$, а также ограниченный и обратимый в $H$ оператор $W$ такие, что

$$
Q_{j}=W P_{j} W^{-1}, \quad j=1,2, \ldots
$$

Доказательство. Докажем, что (i) $\Leftrightarrow$ (ii). Пусть выполнено (ii). Так как билинейная форма в гильбертовом пространстве определяется квадратичной формой, то из (6.4) следует, что

$$
\left|\left(S_{n} x, y\right)\right| \leqslant \sum_{k=1}^{n}\left|\left(Q_{k} x, y\right)\right|<\infty \quad \forall x, y \in H .
$$


Тогда в силу теоремы Банаха-Штейнгауза последовательность $\left\|S_{n} x\right\|$ равномерно ограничена по $n$ при любом $x \in H$. При $x \in S_{n}(H)$ имеем $S_{n} x=x$. Так как подпространства $S_{n}(H)$ образуют плотное множество в $H$, то $S_{n} x \rightarrow x$ при $n \rightarrow \infty$ для любого $x \in H$. Но условие (6.4) выполняется при всех перестановках индексов, поэтому ряд $\sum Q_{j}$ сходится в сильном смысле безусловно. Обратно, если этот ряд сходится в сильном смысле безусловно, то для любых $x, y \in H$ ряд $\sum\left(Q_{k} x, y\right)$ сходится безусловно, а тогда выполнено (6.4).

Отношение эквивалентности (i) $\Leftrightarrow$ (iii) есть хорошо известный результат, к доказательству которого причастны многие математики (см. подробности в [25; гл. 6, теорема 5.1]). В книге [25] соответствующая теорема сформулирована в терминах подпространств $Q_{j}(H)$, но перевод с одного языка на другой не составляет труда. Теорема доказана.

Из теоремы 2.1 получаются полезные достаточные условия. Следующее предложение представляет одну лемму Като [32; лемма V.4.17].

ПРЕДЛОЖЕНИЕ 6.2. Пусть $\left\{Q_{j}\right\}_{i}^{\infty} u\left\{P_{j}\right\}_{1}^{\infty}$ - полные и минимальные системы проекторов, причем $P_{j}=P_{j}^{*}$. Предположим, что

$$
\sum_{j=1}^{\infty}\left\|P_{j}\left(Q_{j}-P_{j}\right) x\right\|^{2} \leqslant q\|x\|^{2} \quad \forall x \in H
$$

при некотором $q<1$ и, кроме того,

$$
\operatorname{dim} Q_{0}=\operatorname{dim} P_{0}=n<\infty .
$$

Тогда существует ограниченный и обратимый оператор $W$ такой, что

$$
Q_{0}=W^{-1} P_{0} W, \quad Q_{j}=W^{-1} P_{j} W, \quad j=1,2, \ldots .
$$

ДокАЗАТЕЛЬство. Идея состоит в следующем. Если оператор преобразования существует, то он имеет вид

$$
W=\sum_{k=0}^{\infty} P_{k} Q_{k}=1-B-P_{0}\left(Q_{0}-P_{0}\right), \quad B=\sum_{k=1}^{\infty} P_{k}\left(Q_{k}-P_{k}\right) .
$$

Действительно, если $W$ имеет такое представление, то, умножая слева на $P_{j}$, а справа на $Q_{j}$, получаем $P_{j} W=Q_{j} W$. Сходимость ряда в сильном смысле гарантируется условием (6.8), причем $\|B\|<1$. Из выписанного представления следует, что $W$ - фредгольмов оператор с нулевым индексом. Поэтому он обратим, если его ядро нулевое. Тривиальность ядра доказывается с помощью равенства (6.7). Предложение доказано.

Приведем еще один важный результат.

Теорема 6.3. Пусть система конечномерных проекторов $\left\{Q_{j}\right\}_{1}^{\infty}$ полна и минимальна, а другая система $\left\{P_{j}\right\}_{1}^{\infty}$ эквивалентна полной и минимальной системе $\left\{P_{j}^{0}\right\}_{1}^{\infty}$ ортогональных проекторов, т.е. $P_{j}=W^{-1} P_{j}^{0} W, j=1,2, \ldots$, где $W$ и $W^{-1}$ ограниченьь. Если

$$
\sum_{j=1}^{\infty}\left\|Q_{j}-P_{j}\right\|^{2}<\infty
$$


то система $\left\{Q_{j}\right\}_{1}^{\infty}$ эквивалентна некоторой полной и минимальной системе ортогональных проекторов $\left\{P_{j}^{\prime}\right\}_{1}^{\infty}$, причем при достаточно больших $j \geqslant j_{0}$ можно положить $P_{j}^{\prime}=P_{j}^{0}$.

ДокАЗАтЕльство. Эта теорема известна как теорема Бари-Маркуса [25; гл. 6 , теоремы 2.3 и 5.2]. Однако сформулирована и доказана она в неудобных терминах: вместо минимальности проекторов $\left\{Q_{j}\right\}_{1}^{\infty}$ требуется $\omega$-линейная независимость подпространств $\left\{Q_{j}(H)\right\}_{1}^{\infty}$. Это условие трудно проверяемое и более жесткое, нежели условие минимальности системы $\left\{Q_{j}\right\}_{1}^{\infty}$. Специалисты (включая автора) подразумевали, что эта теорема верна, если система проекторов на подпространства порождается проекторами Рисса на непересекающиеся части спектра. Но пояснений по этому вопросу автор в литературе не встречал. Поэтому мы приведем доказательство, тем более что на нашем языке оно упрощается.

Если $P_{j}=W^{-1} P_{j}^{0} W$ и $Q_{j}^{1}=W^{-1} Q_{j} W$, то

$$
\sum_{j=1}^{\infty}\left\|Q_{j}^{1}-P_{j}^{0}\right\|^{2} \leqslant\|W\|\left\|W^{-1}\right\| \sum_{j=1}^{\infty}\left\|Q_{j}-P_{j}\right\|^{2}<\infty,
$$

причем новая система проекторов $\left\{Q_{j}^{1}\right\}_{1}^{\infty}$ также полна и минимальна. Поэтому, не ограничивая общности, можно считать, что $\left\{P_{j}\right\}_{1}^{\infty}-$ полная и минимальная система ортогональных проекторов.

Рассмотрим оператор

$$
1+B=1+\sum_{j=n}^{\infty}\left(Q_{j}-P_{j}\right) P_{j}=\sum_{k=n}^{\infty} Q_{j} P_{j}-\sum_{j=1}^{n-1} P_{j} .
$$

Этот ряд сходится в сильном смысле, причем

$$
\begin{aligned}
\|B x\|^{2} & \leqslant \sum_{j=n}^{\infty}\left(\left\|Q_{j}-P_{j}\right\|\left\|P_{j} x\right\|\right)^{2} \\
& \leqslant \sum_{j=n}^{\infty}\left\|Q_{j}-P_{j}\right\|^{2} \sum_{j=n}^{\infty}\left\|P_{j} x\right\|^{2} \leqslant\left(\sum_{j=n}^{\infty}\left\|Q_{j}-P_{j}\right\|^{2}\right)\|x\|^{2} .
\end{aligned}
$$

Выберем число $n$ так, чтобы было выполнено неравенство $\|B\|<1$. Тогда $\left\|Q_{j}-P_{j}\right\|<1$ при $j \geqslant n$. Обозначим

$$
\mathscr{M}_{j}=P_{j}(H), \quad \mathscr{N}_{j}=Q_{j}(H) .
$$

В силу леммы Сёкефальви-Надя [57; гл. 1 , лемма 3.1$]$ проектор $Q_{j}$ при $j \geqslant n$ изоморфно отображает пространство $\mathscr{M}_{j}$ на $\mathscr{N}_{j}$.

Обозначим через $\mathscr{M}$ (соответственно $\mathscr{N}$ ) линейную оболочку подпространств $\mathscr{M}_{1}, \ldots, \mathscr{M}_{n-1}\left(\right.$ соответственно $\left.\mathscr{N}_{1}, \ldots, \mathscr{N}_{n-1}\right)$, а через $\widehat{\mathscr{M}}($ соответственно $\widehat{\mathscr{N}})$ замкнутую линейную оболочку остальных подпространств $\mathscr{M}_{n}, \mathscr{M}_{n+1}, \ldots$ (соответственно $\left.\mathscr{N}_{n}, \mathscr{N}_{n+1}, \ldots\right)$. Очевидно, что

$$
\mathscr{M} \oplus \widehat{\mathscr{M}}=H .
$$


Покажем, что

$$
\mathscr{N}+\widehat{\mathscr{N}}=H .
$$

Сумма подпространств $\mathscr{N}$ и $\widehat{\mathscr{N}}$ содержит все подпространства $\left\{\mathscr{N}_{j}\right\}_{1}^{\infty}$. Из определения полноты системы проекторов следует, что не существует ненулевого вектора, ортогонального всем этим подпространствам, поэтому их сумма плотна в пространстве $H$. Так как $\mathscr{N}$ конечномерно, а $\widehat{\mathscr{N}}$ замкнуто, то $\mathscr{N}+\widehat{\mathscr{N}}=H$. Далее, если сумма не является прямой, то найдется вектор $x \in \mathscr{N} \cap \widehat{\mathscr{N}}, x \neq 0$. Из условия $x \in \mathscr{N}($ соответственно $x \in \widehat{\mathscr{N}})$ следует, что найдется индекс $j, 1 \leqslant j \leqslant n-1$ (соответственно $s \geqslant n$ ) такой, что $Q_{j} x \neq 0$ (соответственно $Q_{s} x \neq 0$ ). Но тогда в силу минимальности системы проекторов имеем $0=Q_{s} Q_{j} x=Q_{s}^{2} x=Q_{s} x \neq 0$. Противоречие. Поэтому сумма $(6.11)$ прямая. Из представления (6.9) следует, что $(1+B)(\widehat{\mathscr{M}})=\widehat{\mathscr{N}}$. Следовательно, $H=\mathscr{N}^{\prime} \dot{+} \widehat{\mathscr{N}}$, где $\mathscr{N}^{\prime}=(1+B)(\mathscr{M})$. Сравнивая с $(6.11)$, получаем, что $\operatorname{dim} \mathscr{N}=\operatorname{dim} \mathscr{N}^{\prime}=\operatorname{dim} \mathscr{M}$. Поэтому подпространство $\mathscr{M}$ можно представить в виде суммы

$$
\mathscr{M}=\mathscr{M}_{1}^{\prime} \oplus \cdots \oplus \mathscr{M}_{n-1}^{\prime},
$$

где $\operatorname{dim} \mathscr{M}_{j}^{\prime}=\operatorname{dim} Q_{j}, j=1, \ldots, n-1$. Пусть $P_{j}^{\prime}, j=1, \ldots, n-1,-$ ортопроекторы на подпространства $\mathscr{M}_{j}^{\prime}$. На этих подпространствах определим оператор $W$ так, чтобы выполнялось равенство $W\left(\mathscr{M}_{j}^{\prime}\right)=Q_{j}$, а на $\widehat{\mathscr{M}}$ положим $W=1+B$. Тогда $W$ обратим в $H$ и преобразует ортогональные подпространства

$$
\mathscr{M}_{1}^{\prime}, \mathscr{M}_{2}^{\prime}, \ldots, \mathscr{M}_{n-1}^{\prime}, \mathscr{M}_{n}, \mathscr{M}_{n+1}, \ldots
$$

в подпространства $\mathscr{N}_{1}, \mathscr{N}_{2}, \ldots$ Теорема доказана.

ЗАМЕЧАНИЕ 6.4. Если известно, что

$$
\operatorname{dim} Q_{j}=\operatorname{dim} P_{j}, \quad j=1,2, \ldots,
$$

то от системы проекторов $\left\{Q_{j}\right\}_{1}^{\infty}$ достаточно требовать только что-то одно: либо полноту, либо минимальность. Это легко усмотреть из доказательства теоремы.

Базисы, которые порождаются системами проекторов, квадратично близкими к полным и минимальным системам ортогональных проекторов, часто называют базисами Бари. Такие базисы выделяют в особый класс, мотивируя это следующим обстоятельством: оператор $W$, реализующий преобразования систем проекторов

$$
W Q_{j}=P_{j} W, \quad j=1,2, \ldots,
$$

в этом случае допускает представление $W=1+K$, где $K$ принадлежит идеалу операторов Гильберта-Шмидта $\mathfrak{S}_{2}$. Это доказано в $[25 ;$ гл. $6, \S 3.1]$ для случая, когда $\operatorname{dim} Q_{j}=\operatorname{dim} P_{j}=1$ при всех $j \geqslant 1$. Утверждение остается справедливым, если

$$
\sup _{j} \operatorname{dim} Q_{j}<\infty .
$$

Если это условие не выполнено, то свойство $K \in \mathfrak{S}_{2}$ уже нельзя гарантировать.

Прежде чем сформулировать основную теорему настоящего раздела, приведем одно предложение из теории функций, которое понадобится в дальнейшем. 
ПрЕДЛОЖЕНИЕ 6.5. Пусть $f(\lambda)$ - голоморфная ограниченная функиия в прямоугольнике

$$
\Pi=\{\lambda:|\operatorname{Re} \lambda|<a,|\operatorname{Im} \lambda|<c\} .
$$

Зафиксируем $\tau \in(0,1)$, положим $a^{\prime}=a(1-\tau), c^{\prime}=c(1-\tau)$ и обозначим через $\Pi^{\prime}$ прямоугольник, который определен равенством (6.12) с заменой а и с на а $a^{\prime}$ и $c^{\prime}$. Введем также обозначения

$$
M=\sup _{\lambda \in \Pi} f(\lambda), \quad M^{\prime}=\sup _{\lambda \in \Pi^{\prime}} f(\lambda) .
$$

Тогда справедливы следующие утверждения:

(i) для числа нулей $n_{f}\left(\Pi^{\prime}\right)$ функиии $f(\lambda)$ в прямоуголънике $\Pi^{\prime}$ справедлива оценка

$$
n_{f}\left(\Pi^{\prime}\right) \leqslant C\left(\ln M-\ln M^{\prime}\right) ;
$$

(ii) для любого $\varepsilon \in(0,1)$ найдутся круги с общей суммой радиусов $\leqslant \varepsilon$ такие, что вне объединения $\mathscr{E}$ этих кругов выполняется оценка снизу

$$
\ln |f(\lambda)| \geqslant(\ln \varepsilon)\left(\ln M-\ln M^{\prime}\right)+\ln M^{\prime}, \quad \lambda \in \Pi^{\prime} \backslash \mathscr{E} ;
$$

(iii) если $\gamma$ - отрезок прямой, заключенный в $\Pi^{\prime}$ и не проходящий через нули $f$ в $\Pi^{\prime}$, то изменение аргумента функции $f$ вдоль $\gamma$ подчинено оченке

$$
\left|[\arg f(\lambda)]_{\gamma}\right| \leqslant C\left(\ln M-\ln M^{\prime}\right) .
$$

Постоянные $C$ во всех оценках зависят только от $\tau$ и отношения а/с и не зависят от $f$. При одновременном увеличении или уменвшении сторон прямоугольников П и П' в $\eta$ раз ощенка (6.14) сохраняется с той же постоянной $C$, но исключительное множество кружков нужно брать с общей суммой радиусов $\leqslant \eta \varepsilon$.

ДокАЗАТЕЛЬство. Варианты приведенных утверждений в другой форме можно найти, например, в книге [65]. В сформулированном виде утверждения (i) и (iii) имеются в [58; леммы 1.1 и 1.3], а утверждения (i) и (ii) - в [72; лемма 2.1], [70].

Следующая теорема представляет собой уточнение замечательной теоремы Маркуса и Мацаева [73], [28; гл. 1, §6].

ТЕОРема 6.6. Пусть Т - нормалъный оператор порядка а со спектром, локализованным на конечном числе лучей $\arg \lambda=\theta_{s}, s=1, \ldots, N$. Если оператор $B$ является $p$-подчиненным оператору $T$ при некотором $p \in[0,1)$, причем $\alpha^{-1} \leqslant 1-p$, то система корневых векторов оператора $A=T+B$ образует безусловный базис со скобками. Более того, это утверждение остается верным, если вместо конечности порядка оператора $T$ потребовать выполнения более слабого условия: существует число $b_{1}>b^{\prime}$ такое, что

$$
\varliminf_{r \rightarrow \infty} n_{j}\left(r+b_{1} r^{p}\right)-n_{j}\left(r-b_{1} r^{p}\right)=m<\infty, \quad j=1, \ldots, N .
$$

Здесъ $b^{\prime}$ - точная грань р-подчиненности в (3.3), a $n_{j}(r)$ - функции распределения собственных значений оператора $T$ на лучах $\arg \lambda=\theta_{j}$. 
Доказательство первого утверждения этой теоремы имеется в [28]. Здесь мы сохраняем основные идеи доказательства, приведенного в [28], но меняем форму изложения и проводим некоторые упрощения, добиваясь при этом большей общности. Изложение доказательства разобьем на несколько этапов.

Шаг 1. Сначала заметим, что условие конечности порядка оператора вместе с условием $\alpha^{-1} \leqslant 1-p$ влекут выполнение условия (6.16), причем с произвольно большим числом $b_{1}$ (обратное, конечно, неверно). Доказательство элементарно (см. [28; лемма 6.11]). Поэтому далее доказываем теорему при условии (6.16). На первом этапе мы максимально упростим задачу и будем считать, что оператор $T=T^{*}$ положителен, а условие (6.16) выполнено при $m=0$, т. е. найдутся интервалы $\Delta_{j}=\left(r_{j}-b_{1} r_{j}^{p}, r_{j}+b_{1} r_{j}^{p}\right)$, которые вовсе не содержат собственных значений $\left\{\mu_{k}\right\}$ оператора $T$. Здесь $b_{1}>b^{\prime}$, где $b^{\prime}-$ точная нижняя грань постоянных $b$ в оценке (3.3). Позже мы избавимся от этих предположений. Доказательство проведем на основе следующих представлений, справедливых при $\lambda \notin \sigma(T) \cup \sigma(A)$ :

$$
(A-\lambda)^{-1}=(T-\lambda)^{-1}-G(\lambda), \quad \text { где } \quad G(\lambda)=(A-\lambda)^{-1} B(T-\lambda)^{-1},
$$

и

$$
\begin{aligned}
G(\lambda)= & (T-\lambda)^{-1} M(\lambda) B(T-\lambda)^{-1}, \\
& \text { где } \quad M(\lambda)=(1+S(\lambda))^{-1}, \quad S(\lambda)=B(T-\lambda)^{-1} .
\end{aligned}
$$

Согласно предложению 3.3 спектр оператора $A=T+B$, за исключением конечного числа собственных значений, лежит внутри параболической области $\Pi_{p, h}$, если $h>b^{\prime}$, а вне этой области и круга достаточно большого радиуса выполнена оценка (3.8). Далее считаем, что $h>2 b^{\prime}$, тогда

$$
\|S(\lambda)\| \leqslant \frac{1}{2}, \quad M(\lambda) \leqslant 2, \quad \text { если } \lambda \notin \Pi_{p, h},|\lambda|>r_{0} .
$$

Ниже на шаге 4 мы покажем, что если интервалы $\Delta_{j}$ не содержат собственных значений $\left\{\mu_{k}\right\}$ оператора $T$, то на вертикальных прямых $\operatorname{Re} \lambda=r_{j}$ справедлива оценка $\|S(\lambda)\| \leqslant 1-\varepsilon$ при некотором $\varepsilon>0$, а потому оператор-функция $M(\lambda)$ равномерно ограничена на этих прямых. В частности, эта оператор-функция равномерно ограничена на вертикальных отрезках $\gamma_{j}$, которые проходят через точки $r_{j}$ и соединяют кривые

$$
\Gamma_{ \pm}=\left\{\lambda \in \mathbb{C}: \operatorname{Im} \lambda= \pm h|\lambda|^{p}\right\}
$$

- границы области $\Pi_{p, 2 b}$ в верхней и нижней полуплоскостях.

Обозначим через $Q_{j}$ и $P_{j}$ проекторы Рисса на части спектра операторов $A$ и $T$, которые заключены внутри криволинейных трапеций $\Pi_{j} \subset P_{2 b}$, ограниченных вертикальными отрезками $\gamma_{j-1}$ и $\gamma_{j}$. Сумма ортогональных проекторов $P_{j}$ безусловно сходится к единичному оператору. Система проекторов $\left\{Q_{j}\right\}$, отвечающих за непересекающиеся части спектра, минимальна в $H$ для любого оператора. Поэтому в силу представления (6.17) для доказательства теоремы 
достаточно показать, что система корневых векторов оператора $A$ полна в $H$ и при всех $x \in H$ сходится ряд

$$
\sum_{j=1}^{\infty}\left|\left(Q_{j} x, x\right)\right|, \quad Q_{j}=-\frac{1}{2 \pi i} \int_{\Gamma_{j}} G(\lambda) d \lambda
$$

где $\Gamma_{k}$ - ориентированные границы криволинейных трапеций $\Pi_{j}$, а $\Gamma_{0}$ - граница области, являющейся пересечением круга достаточно большого радиуса $r_{0}$ и полуплоскости $\operatorname{Re} \lambda \leqslant r_{1}$.

В случае конечности порядка оператора $T$ полнота корневых векторов возмущенного оператора $A$ следует из теоремы 4.7. При выполнении более слабого условия (6.16) свойство полноты сохраняется, об этом мы скажем на заключительном шаге 6. Если равномерная ограниченность оператор-функции $M(\lambda)$ на вертикальных отрезках $\gamma_{j}$ уже доказана, то из представления (6.18) получаем, что для завершения доказательства теоремы достаточно доказать сходимость интегралов

$$
\int_{\Gamma_{ \pm}}\left\|(T-\lambda)^{-1} x\right\|\|S(\lambda) x\||d \lambda|<\infty, \quad x \in H
$$

и ряда

$$
\sum_{j=1}^{\infty} \int_{\gamma_{j}}\left\|(T-\lambda)^{-1} x\right\|\|S(\lambda) x\||d \lambda|<\infty .
$$

Итак, наш план состоит в следующем. На шаге 2 мы оценим оператор-функцию $M(\lambda)$ на вертикальных прямых $\operatorname{Re} \lambda=r_{j}$ при условии, что интервалы $\Delta_{j}$ не содержат собственных значений $\mu_{k}$ оператора $T$, т. е. если выполнено условие (6.16) при $m=0$. Более того, равномерная оценка $M(\lambda)$ будет сохраняться в вертикальных полосах

$$
\Omega_{j}=\left\{\lambda \in \mathbb{C}: r_{j}-\delta r_{j}^{p}<\operatorname{Re} \lambda<r_{j}+\delta r_{j}^{p}\right\}
$$

при любом фиксированном $\delta \in\left(0, b_{1}-b^{\prime}\right)$. Далее мы докажем сходимость интегралов (6.20) и сходимость ряда (6.21), где $\gamma_{j}$ - произвольные вертикальные отрезки в полосах $\Omega_{j}$, соединяющие кривые $\Gamma_{ \pm}$. На шаге 5 с помощью приема искусственной лакуны мы покажем, что если выполнено условие (6.16) с произвольным числом $m \in \mathbb{N}$, то в полосе $\Omega_{j}$ можно выбрать вертикальные отрезки $\gamma_{j}^{\prime}$, на которых нужные оценки сохраняются. Тем самым доказательство будет завершено для случая $T=T^{*}>0$. Изменения, необходимые для доказательства в случае нормального оператора со спектром на конечном числе лучей, мы обсудим на шаге 6.

Шаг 2. Оценим оператор-функцию $S(\lambda)$ на прямой $\operatorname{Re} \lambda=r$ при условии, что интервал $\Delta_{r}=\left(r-b_{1} r^{p}, r+b_{1} r^{p}\right)$ не содержит собственных значений $\mu_{k}$. Здесь и далее, где удобно, пишем $r$ вместо $r_{j}$. Пусть $\lambda=\eta+i \tau$. Из оценки $(2.1)$ получаем

$$
\left\|T(T-\lambda)^{-1}\right\|=\left(\inf _{t \in \sigma(T)}\left|1-\frac{\lambda}{t}\right|\right)^{-1} \leqslant\left(\inf _{t \in \sigma(T)}\left|1-\frac{\eta}{t}\right|\right)^{-1} .
$$


Так как $\sigma(T) \cap \Delta_{j}=\varnothing$, а функция $|1-\eta t-1|$ убывает при $t \in(0, \eta)$ и возрастает при $t \in(\eta, \infty)$, то правая часть (6.23) на прямой $\operatorname{Re} \lambda=r$ не превосходит максимума значений этой функции на концах интервала $\Delta_{j}$, т. е. не превосходит величины

$$
\max \left(\left|1-\frac{r}{r-b_{1} r^{p}}\right|^{-1},\left(1-\frac{r}{r+r^{p}}\right)^{-1}\right)=\frac{r+b_{1} r^{p}}{b_{1} r^{p}}
$$

Используя очевидную оценку $\left\|(T-\lambda)^{-1}\right\| \leqslant\left(b_{1} r^{p}\right)^{-1}$ и оценку (3.3), получаем (далее $o(1)$ есть бесконечно малая величина при $r=r_{j} \rightarrow \infty$ )

$$
\begin{aligned}
\|S(\lambda)\| & \leqslant b\left(\frac{r_{j}+b_{1} r_{j}^{p}}{b_{1} r_{j}^{p}}\right)^{p}\left(\frac{1}{b_{1} r_{j}^{p}}\right)^{1-p}+\frac{M}{b_{1} r_{j}^{p}} \leqslant b\left(\frac{r_{j}+b_{1} r_{j}^{p}}{b_{1} r_{j}^{p}}\right)^{p}\left(\frac{1}{b_{1} r_{j}^{p}}\right)^{1-p}(1+o(1)) \\
& =\frac{b}{b_{1}}(1+o(1))<1,
\end{aligned}
$$

если $b \in\left(b^{\prime}, b_{1}\right)$. Очевидно, что оценка сохраняется в полосе $\Omega_{r}=\Omega_{j}(\delta)$, определяемой формулой (6.22), если $\delta$ достаточно мало. В частности, если $\delta=\left(b_{1}-b^{\prime}\right) / 2$ и $b=b^{\prime}+\delta / 4$, то для $\lambda \in \Omega_{r}, \lambda \gg 1$, получаем

$$
\|S(\lambda)\| \leqslant \frac{b}{b_{1}-\delta}=\frac{2 b}{b_{1}+b^{\prime}}, \quad\|M(\lambda)\| \leqslant \frac{b_{1}+b^{\prime}}{b_{1}+b^{\prime}-2 b}=\frac{2\left(b_{1}+b^{\prime}\right)}{b_{1}-b^{\prime}} .
$$

Шаг 3. Не ограничивая общности, считаем, что оператор $T$ обратим, а тогда из оценки (3.3) следует оценка (3.2) с некоторой новой константой $b$. Оценим интеграл по кривой $\Gamma_{+}$(оценка для $\Gamma_{-}$аналогична). Из (3.2) получаем

$$
\|S(\lambda) x\| \leqslant\left\|T(T-\lambda)^{-1} x\right\|^{p}\left\|(T-\lambda)^{-1} x\right\|^{1-p} .
$$

При $p=0$ ситуация сильно упрощается - нужно работать только со вторым множителем. При $p>0$ интеграл в (6.20) мажорируется интегралом

$$
b \int_{\Gamma_{+}}\left(\left\|T(T-\lambda)^{-1} x\right\|^{p}|\lambda|^{(p-2) p / 2}\right)\left(|\lambda|^{(2-p) p / 2}\left\|(T-\lambda)^{-1} x\right\|^{2-p}\right)|d \lambda|,
$$

который по неравенству Гёльдера (в качестве гёльдеровских сопряженных чисел берем $\left.q=2 / p, q^{\prime}=2 /(2-p)\right)$ не превосходит

$$
b\left(\int_{\Gamma_{+}}|\lambda|^{p-2}\left\|T(T-\lambda)^{-1} x\right\|^{2}|d \lambda|\right)^{p / 2}\left(\int_{\Gamma_{+}}|\lambda|^{p}\left\|(T-\lambda)^{-1} x\right\||d \lambda|\right)^{(2-p) / 2} .
$$

Обозначим через $E(t)$ спектральную функцию оператора $T$ и положим $e(t)=$ $(E(t) x, x)$. Если $\lambda=\xi+i h \xi^{p} \in \Gamma_{+}$и $|\lambda| \geqslant 1$, то

$|\lambda|^{p}=\xi^{p}(1+o(1)), \quad|d \lambda|=d \xi(1+o(1)), \quad|t-\lambda|^{2}=|t-\xi|^{2}+i h^{2} \xi^{2 p}, \quad \xi \geqslant 1$,

поэтому сходимость интегралов в (6.27) эквивалентна сходимости следующих интегралов:

$$
\begin{aligned}
& \int_{1}^{\infty} \int_{0}^{\infty} \frac{\xi^{p} d e(t)}{(t-\xi)^{2}+h^{2} \xi^{2 p}} d \xi=\int_{1}^{\infty} F_{1}(t) d e(t), \quad F_{1}(t)=\int_{1}^{\infty} \frac{\xi^{p}}{(t-\xi)^{2}+h^{2} \xi^{2 p}} d \xi \\
& \int_{1}^{\infty} \int_{0}^{\infty} \frac{t^{2} \xi^{p-2} d e(t)}{(t-\xi)^{2}+h^{2} \xi^{2 p}} d \xi=\int_{1}^{\infty} F_{1}(t) d e(t), \quad F_{2}(t)=\int_{1}^{\infty} \frac{t^{2} \xi^{p-2}}{(t-\xi)^{2}+h^{2} \xi^{2 p}} d \xi
\end{aligned}
$$


Так как функция $e(t)$ монотонна и $0 \leqslant e(t) \leqslant\|x\|^{2}$, то выписанные интегралы сходятся, если функции $F_{1}$ и $F_{2}$ ограничены. Заметим, что

$$
F_{1}(t) \geqslant F_{2}(t), \quad \text { если } t \geqslant \xi, \quad F_{1}(t) \leqslant F_{2}(t), \quad \text { если } t \leqslant \xi .
$$

Поэтому

$$
F_{1}(t)+F_{2}(t) \leqslant 2 \int_{1}^{t} \frac{t^{2} \xi^{p-2}}{(t-\xi)^{2}+h^{2} \xi^{2} p} d \xi+2 \int_{t}^{\infty} \frac{\xi^{p}}{(t-\xi)^{2}+h^{2} \xi^{2} p} d \xi=: 2 I_{1}+2 I_{2} .
$$

Оценка последних интегралов очевидна. Например, первый интеграл оценивается следующим образом:

$$
\begin{aligned}
I_{1} & \leqslant\left(\int_{1}^{t / 2}+\int_{t / 2}^{t-t^{p}}+\int_{t-t^{p}}^{t}\right) \frac{t^{2} \xi^{p-2}}{(t-\xi)^{2}+h^{2} \xi^{2 p}} d \xi \\
& \leqslant \int_{1}^{t / 2} \frac{t^{2} \xi^{p-2}}{(t-\xi)^{2}} d \xi+\int_{t / 2}^{t-t^{p}} \frac{t^{p}}{(t-\xi) 2} d \xi+\int_{t-t^{p}}^{t} \frac{t^{p}}{\xi^{2 p}} d \xi<\frac{4}{1-p}+1+1 .
\end{aligned}
$$

Аналогично оценивается второй интеграл $I_{2}$. Тем самым оценка (6.20) доказана.

Шаг 4. Докажем сходимость ряда (6.21). Так как интервалы $\Delta_{n}$ и $\Delta_{n+1}$ не пересекаются, то $r_{n+1} \geqslant r_{n}+b_{1}\left(r_{n}^{p}+r_{n+1}^{p}\right)$. Поэтому

$$
r_{n+1}^{1-p} \geqslant r_{n}^{1-p}\left(1+2 b_{1} r_{n}^{p-1}\right)^{1-p}>r_{n}^{1-p}+b_{1}(1-p), \quad \text { если } n \geqslant n_{0} .
$$

Следовательно, $r_{n+1}^{1-p}-r_{n}^{1-p}>b_{1}(1-p)$, если $n \geqslant n_{0}$. Тогда по индукции при $j \geqslant n$ получаем

$$
r_{j}^{1-p}-r_{n}^{1-p}>c(j-n), \quad c=b_{1}(1-p), \quad j \geqslant n \geqslant n_{0} .
$$

Поэтому $r_{j}-r_{n} \geqslant r_{j}^{p}\left(r_{j}^{1-p}-r_{n}^{1-p}\right) \geqslant c r_{j}^{p}(j-n), j \geqslant n \geqslant n_{0}$. Меняя $j$ и $n$ местами, получаем

$$
\left|r_{j}-r_{n}\right| \geqslant c \max \left(r_{j}^{p}, r_{n}^{p}\right)|j-n| .
$$

Подынтегральная функция в (6.21) с учетом неравенства (3.3) оценивается величиной

$$
\left\|T(T-\lambda)^{-1} x\right\|^{p}\left\|(T-\lambda)^{-1} x\right\|^{2-p}+M\left\|(T-\lambda)^{-1} x\right\|^{2} .
$$

Здесь главную роль играет первое слагаемое, а второе есть $о(1)$ при $\lambda \rightarrow \infty$ в сравнении с первым. Поэтому интегралы в (6.21) оцениваются интегралами

$$
\int_{\gamma_{j}}\left(\left\|T(T-\lambda)^{-1} x\right\|^{p} r_{j}^{p(p-2) / 2}\right)\left(r_{j}^{p(2-p) / 2}\left\|(T-\lambda)^{-1} x\right\|^{2-p}\right)|d \lambda| .
$$

Теперь, чтобы оценить ряд в (6.21), применим сначала неравенство Гёльдера для интегралов, а затем неравенство Гёльдера для сумм. В результате получим, 
что ряд в (6.21) не превосходит величины

$$
\begin{aligned}
& \sum_{j=1}^{\infty}\left(\int_{\gamma_{j}} r_{j}^{p-2}\left\|T(T-\lambda)^{-1} x\right\|^{2}|d \lambda|\right)^{p / 2}\left(\int_{\gamma_{j}} r_{j}^{p}\left\|(T-\lambda)^{-1} x\right\|^{2}|d \lambda|\right)^{2(2-p)} \\
& \leqslant\left(\sum_{j=1}^{\infty} \int_{\gamma_{j}} r_{j}^{p}\left\|(T-\lambda)^{-1} x\right\|^{2}|d \lambda|\right)^{p / 2}\left(\sum_{j=1}^{\infty} \int_{\gamma_{j}} r_{j}^{p-2}\left\|T(T-\lambda)^{-1} x\right\|^{2}|d \lambda|\right)^{2 /(2-p)} .
\end{aligned}
$$

Оценим первый множитель. Обозначим $x_{k}=\left(x, \varphi_{k}\right)$. При $\lambda \in \gamma_{j}$ имеем

$$
\left\|(T-\lambda)^{-1} x\right\|^{2}=\left\|\sum_{k=1}^{\infty} \frac{x_{k} \varphi_{k}}{\mu_{k}-\lambda}\right\| \leqslant \sum_{k=1}^{\infty} \frac{\left|x_{k}\right|^{2}}{\left|\mu_{k}-r_{j}\right|^{2}} .
$$

Длина отрезка $\gamma_{j}$ не превышает $2 h r_{j}^{p}$, поэтому первый множитель в (6.30) оценивается рядом

$$
\sum_{k=1}^{\infty}\left|x_{k}\right|^{2}\left(\sup _{k} \sum_{j=1}^{\infty} \frac{r_{j}^{2 p}}{\left(\mu_{k}-r_{j}\right)^{2}}\right), \quad \sum_{k=1}^{\infty}\left|x_{k}\right|^{2}=\|x\|^{2} .
$$

Достаточно показать, что супремум здесь ограничен. Найдется число $n \in \mathbb{N}$ такое, что $\mu_{k} \in\left[r_{n}, r_{n+1}\right)$, при этом $r_{n}+b_{1} r_{n}^{p} \leqslant \mu_{k} \leqslant r_{n+1}-b_{1} r_{n+1}^{p}$. Принимая во внимание оценку (6.29), получаем

$$
\sum_{j=1}^{\infty} \frac{r_{j}^{2 p}}{\left(\mu_{k}-r_{j}\right)^{2}} \leqslant \frac{1}{b_{1}^{2}}+\frac{1}{c^{2}}\left(\sum_{j=1}^{n-1}+\sum_{j=n+1}^{\infty}\right) \frac{1}{(n-j)^{2}}<C .
$$

Здесь мы учли, что конечное число слагаемых в сумме при $n \leqslant n_{0}$, для которых (6.29) выполняется с другой константой, на оценку не влияет.

Оценим второй множитель в (6.30). При $\lambda \in \gamma_{j}$ с учетом того, что длина $\gamma_{j}$ не превосходит $2 h r_{j}^{p}$, находим мажоранту этого множителя:

$$
\sum_{j=1}^{\infty} r_{j}^{2 p-2} \sum_{k=1}^{\infty} \frac{\mu_{k}^{2}\left|x_{k}\right|^{2}}{\left(\mu_{k}-r_{j}\right)^{2}}=\sum_{k=1}^{\infty}\left|x_{k}\right|^{2}\left(\sup _{k} \sum_{j=1}^{\infty} \frac{r_{j}^{2 p-2} \mu_{k}^{2}}{\left(\mu_{k}-r_{j}\right)^{2}}\right) .
$$

Остается доказать ограниченность супремума. Фиксируем индекс $k$. Разбивая сумму под знаком супремума на две части, получаем

$$
\left(\sum_{2 r_{j}>\mu_{k}}+\sum_{2 r_{j} \leqslant \mu_{k}}\right) \frac{r_{j}^{2 p-2} \mu_{k}^{2}}{\left(\mu_{k}-r_{j}\right)^{2}} \leqslant 4 \sum_{j} \frac{r_{j}^{2 p}}{\left(\mu_{k}-r_{j}\right)^{2}}+4 \sum_{j} r_{j}^{2(p-1)} \text {. }
$$

Первая сумма уже была оценена. Вторая сумма конечна, но число слагаемых растет при $\mu_{k} \rightarrow \infty$. Для ее оценки воспользуемся неравенством (6.28). Получаем

$$
r_{j}^{1-p} \geqslant c\left(j-j_{0}\right) \quad \text { при } j \geqslant j_{0},
$$

где $j_{0}$ - достаточно большое число. Эта оценка обеспечивает сходимость второго ряда. Тем самым доказана сходимость ряда (6.21). 
Шаг 5. Теперь усложним задачу. Пусть выполнено условие (6.16), т. е. каждый интервал $\Delta_{r}=\left(r-b_{1} r^{p}, r+b_{1} r^{p}\right), r=r_{j}$, содержит $\leqslant m$ собственных значений $\mu_{k}$ оператора $T$. Тогда интегралы по кривым $\Gamma_{ \pm}$оцениваются так же, как прежде, но интегралы по вертикальным отрезкам $\gamma_{j}$ оценить тем же способом не получится, поскольку ограниченность оператор-функции $M(\lambda)$ уже нельзя гарантировать. Оценки нужно проводить по-другому. Идея состоит в следующем. Нужно показать, что в полосе $\Omega_{r}$ ширины $2 \delta$, определяемой формулой (6.22), найдется полоса $\Omega_{r}^{\prime}$ ширины $\delta^{\prime} r^{p}=\delta r^{p} / C$, в которой выполняется оценка

$$
\|F(\lambda)\| \leqslant C_{1}, \quad \text { где } \quad F(\lambda)=B(A-\lambda)^{-1}, \quad \lambda \in \Omega_{r}^{\prime} \subset \Omega_{r} .
$$

Предположим, что это уже доказано. Воспользуемся равенством

$$
(A-\lambda)^{-1}=(T-\lambda)^{-1}(1-F(\lambda)) .
$$

Тогда

$$
\begin{aligned}
|(G(\lambda) x, x)| & =\left|\left((T-\lambda)^{-1}(1-F(\lambda)) S(\lambda) x, x\right)\right| \\
& \leqslant\left(1+C_{1}\right)\left\|(T-\lambda)^{-1} x\right\|\|S(\lambda) x\| \\
& \leqslant\left(1+C_{1}\right)\left\|T(T-\lambda)^{-1} x\right\|^{p}\left\|(T-\lambda)^{-1} x\right\|^{2-p} .
\end{aligned}
$$

Здесь мы воспользовались также оценкой (6.25) и равенством

$$
\left\|(T-\lambda)^{-1} x\right\|=\left\|\left(T^{*}-\bar{\lambda}\right)^{-1} x\right\|,
$$

которое справедливо для нормальных операторов. Пусть $\Delta_{r}^{\prime}=\Omega_{r}^{\prime} \cap \mathbb{R}$. На этом отрезке находится $\leqslant m$ точек $\mu_{k}$, поэтому найдется точка $r^{\prime} \in \Delta_{r}^{\prime}$ такая, что $\operatorname{dist}\left(r^{\prime}, \sigma(T)\right) \geqslant r^{p} \delta^{\prime} /(2 m)$. Пусть $\gamma^{\prime}-$ вертикальные отрезки, проходящие через точки $r^{\prime}$. Тогда интегралы по $\gamma^{\prime}$ от функции (6.33) оцениваются точно так же, как на шаге 4 . Разница лишь в том, что вместо слагаемого $1 / b_{1}^{2}$ в $(6.31)$ появится слагаемое $m\left(2 m / \delta^{\prime}\right)^{2}$.

Итак, надо доказать (6.32). Воспользуемся приемом Мацаева создания "искусственной лакуны". Рассмотрим оператор

$$
K_{r}:=\sum_{\mu_{k}<r}\left(\mu_{k}-r^{-}\right)\left(\cdot, \varphi_{k}\right) \varphi_{k}-\sum_{\mu_{k} \geqslant r}\left(\mu_{k}-r^{+}\right)\left(\cdot, \varphi_{k}\right) \varphi_{k}, \quad r^{ \pm}:=r \pm b_{1} r^{p},
$$

где в суммах участвуют только те индексы $k$, для которых $\mu_{k} \in \Delta_{r}$. Размерность этого оператора не превышает $m$. Положим $T_{r}:=T-K_{r}$. Этот оператор остается нормальным, сохраняет систему собственных векторов $\left\{\varphi_{k}\right\}_{1}^{\infty}$, но меняет собственные значения, лежащие в интервале $\Delta_{r}$, сдвигая их из этого интервала в ближайший из его концов $r^{+}$или $r^{-}$.

Воспользуемся равенством

$$
B(A-\lambda)^{-1}=B\left(T_{r}+B-\lambda\right)^{-1}\left(1+S_{r}(\lambda)\right)^{-1}, \quad S_{r}(\lambda)=B\left(T_{r}-\lambda\right)^{-1} .
$$

Далее оценим множители в этом представлении. Имеем

$$
\left(T_{r}+B-\lambda\right)^{-1}=\left(T_{r}-\lambda\right)^{-1}\left(1+K_{r}\left(T_{r}+B-\lambda\right)^{-1}\right) .
$$


Заметим, что при $\lambda \in \Omega_{r}$

$$
\left\|S_{r}(\lambda)\right\| \leqslant\left\|T\left(T_{r}-\lambda\right)^{-1}\right\|^{p}\left\|\left(T_{r}-\lambda\right)^{-1}\right\|^{1-p} \leqslant\left\|T T_{r}^{-1}\right\|^{p} \frac{2\left(b_{1}+b^{\prime}\right)}{b_{1}-b^{\prime}} .
$$

Здесь мы воспользовались неравенством (6.24) для оператора $T_{r}$, не имеющего собственных значений в интервале $\left(r_{-}, r_{+}\right)$. Кроме того, $\left\|T T_{r}^{-1}\right\| \leqslant r r_{-}^{-1}=$ $1+o(1)$, так как $r^{-}=r-b_{1} r^{p}$. Следовательно, $\left\|\left(1+S_{r}(\lambda)\right)^{-1}\right\| \leqslant C$ в полосе $\Omega_{r}$, а тогда при $\lambda \in \Omega_{r}$

$$
\begin{aligned}
\left\|\left(T_{r}+B-\lambda\right)^{-1}\right\| & \leqslant \frac{C}{\left(b_{1}-\delta\right) r^{p}} \leqslant C_{1} r^{-p}, \\
\left\|B\left(T_{r}+B-\lambda\right)^{-1}\right\| & \leqslant C\left\|B\left(T_{r}-\lambda\right)^{-1}\right\|=C\left\|S_{r}(\lambda)\right\|<C .
\end{aligned}
$$

Остается оценить оператор-функцию

$$
\left(1+K^{-1}(\lambda)\right)^{-1}, \quad K(\lambda)=1+K_{r}\left(T_{r}+B-\lambda\right)^{-1} .
$$

Из определения оператора $K_{r}$ следует, что $\left\|K_{r}\right\| \leqslant b_{1} r^{p}$. Из (6.36) получаем $\|K(\lambda)\| \leqslant C$. Теперь для оценки оператор-функции (6.37) воспользуемся теоремой 4.1, полагая в ней $\lambda=-1, K=K(\lambda)$. Заметим, что в рассматриваемом случае числитель в правой части равенства (4.3) оценивается величиной $(1+C)^{m}$, так как s-числа оператора $K$ не превышают его нормы. Знаменатель есть скалярная функция $D(\lambda):=\operatorname{det}(1+K(\lambda))-$ произведение $m$ чисел $1+\lambda_{j}(K(\lambda))$, где $\lambda_{j}(K(\lambda))$ - собственные значения $K$ (не следует путать: в теореме 4.1 мы считаем, что $\lambda=-1$, но в рассматриваемом случае $K=K(\lambda)$ - голоморфная оператор-функция в полосе $\Omega_{r}$ ). Собственные значения также оцениваются постоянной $C$, поэтому во всей полосе $\Omega_{r}$ справедлива оценка сверху $|D(\lambda)| \leqslant(1+C)^{m}$. В силу предложения 3.1 имеем $\left\|\left(T_{r}+B-\lambda\right)^{-1}\right\|<C \tau^{-1}$, $\tau=\operatorname{Im} \lambda$. Возьмем $h=2\left(C b_{1}\right)^{-1}$. Тогда $\|K(r+i h)\| \leqslant 1 / 2$, поэтому модули всех собственных значений оператора $K(r+i h)$ не превосходят $1 / 2$. Следовательно, имеется оценка снизу $|D(r+i h)|>(1-1 / 2)^{m}=2^{-m}$.

Далее воспользуемся предложением 6.2 для оценки функции $D$. В качестве прямоугольника П возьмем прямоугольник с центром в точке $r$, высотой $4 h$ и шириной $2 \delta$, а в качестве $\Pi^{\prime}$ - вдвое меньший прямоугольник с тем же центром. Функция $D$ в прямоугольнике П ограничена постоянной $M=(1+c)^{m}$, а для постоянной $M^{\prime}$ в прямоугольнике $\Pi^{\prime}$ мы получили оценку снизу $M^{\prime} \geqslant$ $2^{-m}$. Согласно предложению 6.2 во всем прямоугольнике $\Pi^{\prime}$ вне исключительного множества кружков с общей суммой радиусов $\varepsilon r^{p}$ функция $D$ оценивается снизу некой постоянной. Кроме того, число нулей функции $D$ в прямоугольнике $\Pi^{\prime}$ не превышает $C m$. Поэтому $\varepsilon$ можно выбрать столь малым, чтобы полоса $\Pi^{\prime \prime}$ ширины $c r^{p}$ в прямоугольнике $\Pi^{\prime}$ при некотором достаточно малом $c>0$ не пересекала исключительное множество. Тогда в этой полосе $\Pi^{\prime \prime}$ оператор-функция $(1+K(\lambda))^{-1}$ (а потому и функция $B(A-\lambda)^{-1}$ ) равномерно ограничена постоянной, не зависящей от $r=r_{j} \rightarrow \infty$. Этим заканчивается доказательство нужных оценок. 
Шаг 6. Мы рассмотрели случай $T=T^{*}>0$. Это условие эквивалентно тому, что $T$ - нормальный оператор со спектром на одном луче. Случай, когда спектр нормального оператора $T$ локализован не на одном, а на нескольких лучах $\arg \lambda=\theta_{j}, j=1, \ldots, N$, не вносит трудностей. Нужно взять окружность $\Gamma$ достаточно большого радиуса, не пересекающую собственных значений $\left\{\mu_{k}\right\}$ и $\left\{\lambda_{k}\right\}$ операторов $T$ и $A=T+B$ соответственно, и рассмотреть области $\Pi_{j}=e^{i \theta_{j}} \Pi_{p, h}$, получающиеся поворотом области $\Pi_{p, h}$. В каждой из областей $\Pi_{j}$ можно провести точно такие же рассуждения, как прежде: найти отрезки, соединяющие границы параболической области $\Pi_{j}$, на которых будут выполняться "хорошие" оценки резольвенты $(A-\lambda)^{-1}$. Нужно только заметить, что собственные значения $\mu_{k}$, которые лежат вне рассматриваемой области $\Pi_{j}$, являются “далекими” и на выполнение оценок никак не влияют. Тем самым мы покажем, что корневые векторы оператора $A$, отвечающие собственным значениям из рассматриваемой области $P_{j}$, образуют безусловный базис со скобками в замыкании своей линейной оболочки. Это верно для всех $j=1, \ldots, N$, поэтому тем же свойством обладает система всех корневых векторов оператора $A$. Остается заметить, что эта система полна в пространстве $H$. Действительно, если полноты нет, то найдется вектор $0 \neq x \in H$ такой, что скалярная функция $F(\lambda)=\left(A-\lambda^{-1} y, x\right)$ является целой при любом $y \in H$. Заметим, что вне парабол $\Pi_{p, h}$ функция $F$ убывает, а внутри парабол найдутся уходящие на бесконечность отрезки, на которых она также ограничена. Из принципа максимума модуля получаем $F(\lambda) \equiv 0$. Но тогда $x=0$. Этим завершается доказательство самой теоремы 6.6.

ЗАмЕчАниЕ 6.7. Пусть $\alpha$ - порядок оператора $T$, а $n(t)$ - функция распределения его собственных значений. Для простоты считаем, что $T>0$. Тогда $n(t)=O\left(t^{\beta}\right), \beta=\alpha^{-1}$. Положим $p=1-\beta$ и при фиксированном $b>0$ рассмотрим функцию

$$
R(t)=n\left(t+b t^{p}\right)-n\left(t-b t^{p}\right)
$$

и множество

$$
\Omega=\left\{t \in \mathbb{R}_{+}: R(t) \leqslant m\right\} .
$$

Легко показать [28; гл. 1, лемма 6.11], что при достаточно большом $m=m(b)$ это множество неограничено. Назовем его зоной несгущаемости спектра опеpamopa T. Из доказательства теоремы следует, что скобки нужно расставлять в зонах несгущаемости спектра, причем в самих зонах несгущаемости в скобки нужно объединять только корневые векторы, которые отвечают сближающимся собственным значениям, $\left|\mu_{k}-\mu_{j}\right|=o(1)$ при $k \rightarrow \infty$. В частности, если $R(t)=O(1)$ (это условие часто выполняется для обыкновенных дифференциальных операторов), то $\Omega=\mathbb{R}_{+}$при достаточно большом $m$ и в скобки нужно объединять не более конечного числа членов, отвечающих сближающимся собственным значениям.

ЗАмЕчАниЕ 6.8. В приведенном доказательстве в качестве контуров интегрирования $\Gamma_{j}$ мы выбирали криволинейные трапеции, стороны которых ограничены параболами $\Gamma_{ \pm}$. Полезно иметь в виду, что контуры $\Gamma_{k}$ можно выбрать 
по-другому. Вместо парабол $\Gamma_{ \pm}$возьмем лучи

$$
\Gamma_{ \pm}^{\prime}=\left\{\lambda: \lambda=e^{ \pm i \delta} r, r>0\right\}
$$

где $\delta$ столь мало, что в секторе между лучами $\Gamma_{ \pm}^{\prime}$ спектр оператора $T$ состоит только из вещественных собственных значений. Заметим, что на лучах $\Gamma_{ \pm}^{\prime}$ имеет место оценка $\|G(\lambda)\| \leqslant C|\lambda|^{p-2}$, что следует из оценки (3.2) и представления (6.18). Поэтому оценка интеграла от функции $|(G(\lambda) x, x)|$ по лучам $\Gamma_{ \pm}^{\prime}$ тривиальна. А тогда вместо оценок по параболам достаточно доказать, что сходится ряд

$$
\sum_{j} \int_{l_{j}^{ \pm}}\left\|T(T-\lambda)^{-1} x\right\|^{p}\left\|(T-\lambda)^{-1} x\right\|^{2-p}|d \lambda|,
$$

где $l_{j}^{ \pm}$- вертикальные отрезки, соединяющие параболы $\Gamma_{ \pm}$и лучи $\Gamma_{ \pm}^{\prime}$, которые являются продолжением отрезков $\gamma_{j}^{\prime}$.

Можно получить аналог теоремы Маркуса-Мацаева для возмущений нормальных операторов $p$-подчиненными квадратичными формами. Это было сделано М. С. Аграновичем в работе [60] для операторов $T=T^{*} \gg 0$. Результаты раздела 2 позволяют получить обобщение для нормальных операторов, в частности для произвольных самосопряженных.

Теорема 6.9. Пусть $T$ - нормальный оператор порядка а со спектром, локализованным на конечном числе лучей $\arg \lambda=\theta_{j}, j=1, \ldots, N$. Если оператор $B$ порождается $p$-подчиненной оператору $T$ формой при некотором $p \in[0,1)$, причем $\alpha^{-1} \leqslant 1-p$, то система корневых векторов оператора $A=T+B$ образует безусловный базис со скобками.

ДокАЗАТЕЛьство. Мы не будем проводить здесь полное доказательство, укажем лишь основные моменты (см. детали в [60]). Отметим, что "скопировать" доказательство теоремы 6.6 не удается, оценки нужно проводить по-другому. Определение суммы операторов $T+B$ и оценки резольвенты возмущенного оператора вне параболических областей даны в разделе 3. Рассуждения, приведенные на шаге 6 в теореме 6.6, позволяют свести доказательство к случаю $T>0$.

Вместо (6.17) воспользуемся следующим представлением:

$$
(A-\lambda)^{-1}-(T-\lambda)^{-1}=V(\lambda)\left(1+S_{1}(\lambda)\right)^{-1} S_{1}(\lambda) V(\lambda)=: G_{1}(\lambda),
$$

где $V(\lambda)$ и $S_{1}(\lambda)$ те же, что в $(2.12)$ и (2.13). Оценку функции $S_{1}(\lambda)$ внутри параболической области проводим так же, как в предложении 3.2. А именно, в силу (3.6) (для простоты полагаем $M=0$ ) справедлива оценка

$$
\begin{aligned}
\left|\left(S_{1}(\lambda) x, x\right)\right| & \leqslant b\left\|T^{1 / 2}(T-\lambda)^{-1 / 2} x\right\|^{2 p}\left\|(T-\lambda)^{-1 / 2} x\right\|^{2-2 p} \\
& =b \sup _{t \in \sigma(T)}\left|\frac{t^{1 / 2}}{|t-\lambda|^{1 / 2}}\right|^{2 p} \sup _{t \in \sigma(T)}\left(\frac{1}{|t-\lambda|^{1 / 2}}\right)^{2-2 p} \\
& =\sup _{t \in \sigma(T)}\left|\frac{t}{t-\lambda}\right|^{p} \sup _{t \in \sigma(T)}\left|\frac{1}{t-\lambda}\right|^{1-p} .
\end{aligned}
$$


Поэтому $\left\|S_{1}(\lambda)\right\|$ оценивается удвоенной величиной, фигурирующей в правой части. Это позволяет провести оценки интегралов от функции $\left|\left(G_{1}(\lambda) x, x\right)\right|$ по вертикальным отрезкам $\gamma_{j}^{\prime}$ почти так же, как в теореме 6.3. Оценку интегралов по параболам получить не удается, нужно воспользоваться замечанием 6.7 и проводить оценки по вертикальным отрезкам $l_{j}$, соединяющим лучи $\Gamma_{ \pm}^{\prime} \cdot$ Далее доказательство сводится к доказательству сходимости ряда

$$
\sum_{j} \int_{l_{j}}\left|\left(G_{1}(\lambda) x, x\right)\right||d \lambda|, \quad \lambda=r_{j}+i \tau,
$$

где функция $G_{1}(\lambda)$ определена в (6.38). Оценки для функций $V$ и $S_{1}$ известны, а для функции $\left(1+S_{1}(\lambda)\right)^{-1}$ получаются с помощью приема искусственной лакуны (так же, как на шаге 5 в теореме 6.6) с учетом оценки (6.39). Возникает еще один момент. Не удается доказать сходимость последнего ряда при произвольном выборе чисел $r_{n}$ в зонах несгущаемости спектра (числа $r_{n}$ суть точки пересечения отрезков $l_{j}$ с вещественной осью) так, как это сделано в теореме 6.6 (см. замечание 6.7). Однако удается показать, что при выборе достаточно редкой последовательности $r_{n}$ ряд становится сходящимся. Это обстоятельство не дает возможности доказать теорему при более общем условии (6.16) на поведение собственных значений оператора $T$, как это сделано в теореме 6.6.

В заключение этого раздела сделаем несколько замечаний. Теореме 6.3 предшествовал результат А. С. Маркуса [16], полученный при условии сильной $p$-подчиненности, условии конечности порядка и при наличии "больших" лакун в спектре, когда не требуется применения приема искусственной лакуны. Затем В.Э. Кацнельсон [24] доказал безусловную базисность со скобками при выполнении условия (7.3) и условия подчинения (3.4), которое более ограничительно, нежели (3.5) или (3.6). Доказательство в [24] использует совсем другие, весьма элегантные инструменты. А именно, оно использует функциональное исчисление для диссипативных операторов, развитое в работах И. Шура и Дж. фон Неймана, а также интерполяционную теорему Карлесона. При этом полезно отметить следующую деталь. Кацнельсон заметил, что при выполнении условия (3.4) достаточно сначала доказать теорему для случая $p=0$ (мы уже отмечали, что в этом случае доказательства теорем 6.6 и 6.9 значительно упрощаются), а затем для доказательства при $p \in(0,1), \alpha^{-1} \leqslant 1-p$ можно применить следующий трюк. Нужно рассмотреть оператор

$$
(T+B)^{\alpha}=T^{\alpha}+R, \quad R=(T+B)^{\alpha}-T^{\alpha} .
$$

Как мы видели ранее, здесь степени операторов корректно определены (в [24] рассматривался только случай $T>0$ ). Оказывается, что при выполнении условия (3.4) оператор $R$ оказывается ограниченным. Тем самым, вопрос сводится к случаю $p=0$, так как системы корневых векторов операторов $T+B$ и $(T+B)^{\alpha}$ совпадают. K сожалению, попытки применить такой прием при более слабых условиях подчинения нам не удались.

Мы уже отмечали в разделе 3 , что сильные $p$-подчиненные возмущения можно рассматривать и при $p<0$. Задача о базисности в этом случае изучалась в работах М. С. Аграновича [33], [74]. Возмущения отрицательного порядка естественно возникают при рассмотрении псевдодифференциальных операторов. 


\section{7. Локально р-подчиненные возмущения}

В этом разделе мы предполагаем, что невозмущенный оператор $T$ самосопряжен. По прежнему через $\left\{\mu_{k}\right\}$ обозначаем его собственные значения, а через $\left\{\varphi_{k}\right\}$ - полную ортонормированную систему соответствующих собственных векторов. Положительные собственные значения нумеруем индексами $k \geqslant 1$ в порядке возрастания модулей, а отрицательные собственные значения - индексами $k \leqslant 0$. Оператор $B$ назовем локально $p$-подчиненным оператору $T$, если

$$
\varphi_{k} \in \mathscr{D}(B) \quad \text { и } \quad\left\|B \varphi_{k}\right\| \leqslant b\left|\mu_{k}\right|^{p} .
$$

Здесь $-\infty<p<1$. Конечно, условие (7.1) значительно слабее условия обычной $p$-подчиненности. Отличие этого условия от ранее рассмотренных состоит в том, что выполнение оценок требуется не для всех $x \in \mathscr{D}(T) \subset \mathscr{D}(B)$ или $x \in \mathscr{D}\left(T^{1 / 2}\right)$, а только локально на базисных векторах $\varphi_{k}$. Для общих операторов это условие было введено в работах автора [51], [52]. Этим работам предшествовала работа Дж. Аддучи и В. С. Митягина [41] (именно она послужила моделью для общей постановки), в которой была решена задача о безусловной базисности корневых функций для случая, когда $T y=-y^{\prime \prime}+x^{2} y$ - гармонический осциллятор в пространстве $H=L_{2}(\mathbb{R})$, а $B y=q(x) y$-оператор умножения на функцию $q \in L_{2}(\mathbb{R})$. Эта задача не решается с помощью приведенных в разделе 6 теорем. В работе [41] она была решена с помощью неожиданного приема с использованием дискретного преобразования Гильберта.

Полезно определить локальную $p$-подчиненность на формах. Конечно, выполнение условий (3.6) или (3.5) на базисных векторах не дает достаточной информации об операторе $B$. Поэтому вместо условия (3.5) будем исходить из условия

$$
|\mathfrak{b}[x, y]| \leqslant\left\|T^{1 / 2} x\right\|^{p}\left\|T^{1 / 2} y\right\|^{p}\|x\|^{1-p}\|y\|^{1-p} .
$$

Будем говорить, что форма $\mathfrak{b}=\mathfrak{b}[x, x]$ локально р-подчинена оператору $T$, если при некотором $p<1$ выполняется оценка

$$
\varphi_{k} \in \mathscr{D}\left(T^{1 / 2}\right), \quad\left|\mathfrak{b}\left[\varphi_{k}, \varphi_{j}\right]\right| \leqslant b\left|\mu_{k}\right|^{p / 2}\left|\mu_{j}\right|^{p / 2},
$$

где $b$ - некоторая постоянная. Пусть порядок оператора $T$ равен $\alpha$, что эквивалентно условию

$$
\varlimsup_{t \rightarrow \infty} n(t, T) t^{-\beta}=C<\infty, \quad \text { или } \quad\left|\mu_{k}\right| \geqslant C^{-1} k^{\alpha}, \quad \alpha=\beta^{-1} .
$$

Здесь $n(t, T)$ - считающая функция собственных значений оператора $T$ (число собственных значений на отрезке $[-t, t])$. В случае $\alpha=1$ последовательность $\left\{\mu_{k}\right\}$ назовем несгущающейся, если найдется число $l \in \mathbb{N}$ такое, что для всех $t \in \mathbb{R}$ отрезок $[t, t+1]$ содержит не более $l$ чисел $\mu_{k}$ из этой последовательности, т. е. $n(t+1, T)-n(t, T) \leqslant l$. При $\alpha \neq 1$ последовательность $\mu_{k}$ назовем $\alpha$-несгущающейся, если

$$
n\left((t+1)^{\alpha}, T\right)-n\left(t^{\alpha}, T\right) \leqslant l
$$

при некотором $l \in \mathbb{N}$. Это эквивалентно тому, что последовательность $\left\{\left|\mu_{k}\right|^{\beta}\right\}$, где $\beta=\alpha^{-1}$, является несгущающейся. Очевидно, что если $\left\{\mu_{k}\right\}$ является $\alpha$-несгущающейся, то выполнено условие (7.3), но обратное, конечно, неверно. 
Сначала займемся локально подчиненными возмущениями с условием (7.1). Сущность полученного ниже результата в следующем: мы требуем более слабое условие $p$-подчиненности (локального типа), но накладываем более сильное условие на регулярность поведения собственных значений невозмущенного оператора $T$.

Tеорема 7.1. Пусть T- оператор порядка $\alpha$ и оператор $B$ локально р-подчинен оператору $T$ при некотором $p \in[0,1)$, причем $\alpha^{-1} \leqslant 1-p$. Предположим, что выполняется одно из условий:

(i) $\alpha \geqslant 1 u\left\{\mu_{k}\right\}$ является $\alpha$-несгущающейся последовательностью;

(ii) $\alpha>1 / 2$ и существуют числа $l \in \mathbb{N} u \tau>0$ такие, что

$$
\left|\mu_{k+l}-\mu_{k}\right| \geqslant \tau k^{\alpha-1} \text {. }
$$

Тогда корневые векторы оператора $T+B$ образуют базис Рисса со скобками в пространстве $H$. При этом расстановку скобок можно провести так, чтобы в каждом блоке были объединены не более $l_{1}$ корневых векторов, где $l_{1}$ фиксированное число.

ДокАЗАТЕЛьство. Сначала отметим, что эта теорема доказана Дж. Аддучи и В. С. Митягиным [42] в случае, когда вместо условия (7.1) выполняется более сильное условие

$$
\left\|B \varphi_{k}\right\|=o(1)\left|\mu_{k}\right|^{p}, \quad \text { где } o(1) \rightarrow 0 \text { при } k \rightarrow \infty .
$$

Здесь мы проведем доказательство этой теоремы при $\alpha=1$. При $p \in(0,1)$ задача сложнее, и мы рассмотрим ее в другой работе.

Разобьем доказательство теоремы 7.1 при $\alpha=1$ на несколько этапов.

Шаг 1. Наметим план доказательства. Сначала мы покажем, что оператор $T+B$ корректно определен на области $\mathscr{D}(T)$ и система его корневых векторов полна в $H$. Затем докажем, что при достаточно большом $h$ все собственные значения оператора $A=T+B$ лежат в полосе $|\operatorname{Im} \lambda|<h$, стороны которой обозначаются через $\Gamma_{ \pm}=\Gamma_{ \pm}(h)$. Более того, справедлива асимптотика

$$
\begin{gathered}
n_{ \pm}(t, A)=n_{ \pm}(t, T)+O(1), \quad t \rightarrow \infty, \\
\text { или } \quad \lambda_{k}=\mu_{k}+O(1), \quad k \rightarrow \pm \infty,
\end{gathered}
$$

где $\lambda_{k}$ - собственные значения оператора $A=T+B$, а $n_{ \pm}(t, A)$ и $n_{ \pm}(t, T)-$ функции распределения положительных и отрицательных собственных значений операторов $A$ и $T$ соответственно. В частности, последовательность $\lambda_{k}$ оказывается также несгущающейся.

Далее будем использовать представления (6.17) и работать с функциями

$$
G(\lambda)=(T-\lambda)^{-1} M(\lambda) S(\lambda), \quad M(\lambda)=(1+S(\lambda))^{-1}=(T-\lambda)(A-\lambda)^{-1},
$$

где $S(\lambda)=B(T-\lambda)^{-1}$. Идея доказательства состоит в следующем. Так же как в доказательстве теоремы 6.3, нам нужно доказать сходимость ряда (6.21). Сначала работаем с функцией $S$. При достаточно большом $h$ справедлива оценка $\|S(\lambda)\| \leqslant 1 / 2$, поэтому функция $M(\lambda)$ равномерно ограничена вне полосы $\Pi_{h}$, 
границами которой являются прямые $\Gamma_{ \pm}(h)$. Пусть $\mathscr{K}_{T}$ и $\mathscr{K}_{A}-$ множества кружков радиуса $\varepsilon$ с центрами в собственных значениях $\mu_{k}$ и $\lambda_{k}$ операторов $T$ и $A$ соответственно. Мы покажем, что функция $M(\lambda)$ равномерно ограничена во всей комплексной плоскости за исключением множества кружков $\mathscr{K}_{A}$.

Важный момент состоит в доказательстве того, что вектор-функция

$$
F(\lambda)=S(\lambda) x, \quad x \in H,
$$

принадлежит $L_{2}(\mathbb{R}+i h)$ при любом $h \neq 0$. Это тем более верно для вектор-функции $(T-\lambda)^{-1} x$ (достаточно положить $B=1$ ). Предположим, что это уже доказано. Выберем последовательность вертикальных отрезков $\gamma_{k}$, уходящих на $\pm \infty$ при $k \rightarrow \pm \infty$ и имеющих концы на сторонах полосы $\Pi_{h}$, так, чтобы они не проходили через множества кружков $\mathscr{K}_{A}$ и $\mathscr{K}_{T}$. При достаточно малом $\varepsilon$ такие отрезки можно выбрать в каждом сегменте $[k, k+1]$. Тогда из представления для функции $G$ следует, что

$$
\begin{aligned}
\int_{\Gamma_{ \pm}}|(G(\lambda) x, x)||d \lambda|+\sum_{k} \int_{\gamma_{k}}|(G(\lambda) x, x)||d \lambda| \\
\quad \leqslant\left(\int_{\Gamma_{ \pm}}+\sum_{k} \int_{\gamma_{k}}\right)\left(\|S(\lambda) x\|^{2}+M^{2}\left\|(T-\lambda)^{-1} x\right\|^{2}\right)|d \lambda|,
\end{aligned}
$$

где $M=M(\varepsilon)$ - постоянная, которой ограничена функция $M(\lambda)$ вне множества $\mathscr{K}_{A}$. Поэтому теорема будет доказана, если мы докажем сходимость суммы интегралов по отрезкам $\gamma_{k}$ от функции $\|S(\lambda)\|^{2}$.

Основная трудность при реализации этого плана - доказать утверждения $S(\lambda) \in L_{2}(\mathbb{R} \pm i h)$. Мы не видим возможности доказать это с помощью разбиения функции на какие-либо слагаемые и дальнейшей оценки их по модулю. Здесь используются более тонкие инструменты.

Приступим к реализации намеченного плана.

Шаг 2. Из условия несгущаемости последовательности $\mu_{k}$ следует, что порядок оператора $T$ равен 1 , т. е. $\left|\mu_{ \pm k}\right| \geqslant c|k|$. Покажем, что оператор $K=$ $B T^{-1}$, определенный на плотном в $H$ множестве (линейных комбинациях системы $\left.\left\{\varphi_{k}\right\}\right)$, продолжается по непрерывности на все пространство $H$ как компактный оператор.

Пусть вектор $x$ есть линейная комбинация системы $\left\{\varphi_{k}\right\}$. Воспользуемся равенствами

$$
T^{-1}=\sum_{k=-\infty}^{\infty} \frac{\left(\cdot, \varphi_{k}\right) \varphi_{k}}{\mu_{k}}, \quad B T^{-1} x=\sum_{k=-\infty}^{\infty} \frac{x_{k} B \varphi_{k}}{\mu_{k}}, \quad x_{k}=\left(x, \varphi_{k}\right) .
$$

Тогда

$$
\left\|B T^{-1} x-\sum_{k=-N}^{N} \frac{x_{k} B \varphi_{k}}{\mu_{k}}\right\|=\left\|\sum_{|k|>N} \frac{x_{k} B \varphi_{k}}{\mu_{k}}\right\| \leqslant\|x\|^{2}\left\|\sum_{|k|>N} \frac{\left\|B \varphi_{k}\right\|^{2}}{\mu_{k}^{2}}\right\| \leqslant \varepsilon\|x\|^{2},
$$

где $\varepsilon \rightarrow 0$ при $N \rightarrow \infty$, так как ряд в правой части сходится в силу неравенства $\left|\mu_{ \pm k}\right| \geqslant c|k|$. Следовательно, оператор $B T^{-1}$ аппроксимируется с произвольной точностью конечномерными операторами, а потому продолжается на 
все $H$ как компактный оператор. В силу предложения 2.2 оператор $B$ является $T$-подчиненным с точной $T$-гранью 0 . Так как области определения операторов $T+B$ и $T$ совпадают, то эти операторы имеют одинаковые порядки. Согласно следствию 4.11 система корневых векторов оператора $A$ полна в $H$.

Шаг 3. Оценим функцию $S(\lambda)$. Справедливо представление

$$
S(\lambda)=\sum_{k=-\infty}^{\infty} \frac{x_{k} B \varphi_{k}}{\mu_{k}-\lambda}, \quad x_{k}=\left(x, \varphi_{k}\right) .
$$

По условию выполнено неравенство $\left\|B \varphi_{k}\right\| \leqslant b$. Поэтому

$$
\|S(\lambda) x\|^{2} \leqslant b^{2}\left\|\sum_{k} \frac{x_{k}}{\mu_{k}-\lambda}\right\|^{2} \leqslant b^{2}\left(\sum_{k} \frac{1}{\left|\lambda-\mu_{k}\right|^{2}}\right)\|x\|^{2} .
$$

Предположим сначала, что каждый отрезок $[k, k+1]$ содержит не более одного собственного значения $\mu_{k}$. Тогда, полагая $\lambda=\sigma+i \tau$, находим:

$$
\begin{aligned}
\|S(\lambda)\| & \leqslant b^{2} \sum_{k} \frac{1}{\left(\mu_{k}-\sigma\right)^{2}+\tau^{2}} \leqslant b^{2} \sum_{s} \frac{1}{\tau^{2}+s^{2}} \\
& \leqslant 2 b^{2}\left(\frac{1}{\tau^{2}}+\int_{1}^{\infty} \frac{d x}{\tau^{2}+x^{2}}\right)<\frac{b^{2} C}{\tau} .
\end{aligned}
$$

Теперь, очевидно, если в каждом интервале $[k, k+1]$ содержится $\leqslant l$ собственных значений $\mu_{k}$, то оценка сохранится, но постоянная $C$ заменится на $C l$. Поэтому $\|S(\lambda)\|<1 / 2$, если $\tau \geqslant 2 b^{2} C l=h$.

Доказательство асимптотики (7.5) мы здесь проводить не будем. Оно имеется в работе автора [51] для случая $T>0$ (но для общего случая $T=T^{*}$ доказательство не меняется).

Шаг 4. Покажем, что $S(\lambda) x \in L_{2}(\mathbb{R}+i h)$. Это основной момент в доказательстве. Полезно получить интегральное представление ряда (7.8). Рассмотрим гладкую (или кусочно гладкую) функцию $\chi(t)$ с носителем на отрезке $[-1,1]$. Положим, например, $\chi(t)=1-|t|$ при $t \in[-1,1]$ и $\chi(t) \equiv 0$ при $|t| \geqslant 0$. Обозначим через $n_{+}(t)$ и $n_{-}(t)$ считающие функции положительных и отрицательных собственных значений $\left\{\mu_{k}\right\}$ соответственно. Положим

$$
n(t)= \begin{cases}n_{+}(t), & \text { если } t>0, \\ -n_{-}(-t), & \text { если } t<0 .\end{cases}
$$

Рассмотрим вектор-функцию

$$
r(t):=\sum_{k=-\infty}^{\infty} x_{k} \chi\left(t-\mu_{k}\right) B \varphi_{k}, \quad x_{k}=\left(x, \varphi_{k}\right) .
$$

Эта функция корректно определена, так как при каждом $t \in \mathbb{R}$ число слагаемых в этой сумме конечно и не превосходит $2 l$. Более того, $r(t) \in L_{2}(\mathbb{R})$, так как

$$
\int_{\mathbb{R}}\|r(t)\|^{2} d t \leqslant 2 b l \sum\left|x_{k}\right|^{2} \int_{\mathbb{R}}\left|\chi\left(t-\mu_{k}\right)\right|^{2} d t<b l\|x\|^{2} .
$$


Очевидно, что вектор-функцию (7.8) можно переписать в интегральной форме

$$
S(\lambda)=\int_{\mathbb{R}} \frac{r(t) d n(t)}{t-\lambda}
$$

Для функции $n(t)$ докажем следующее представление:

$$
n(t)=\psi(t)+\eta(t), \quad\left|\psi^{\prime}(t)\right| \leqslant l, \quad|\eta(t)| \leqslant l,
$$

где $\psi$ - кусочно линейная непрерывная функция, а число $l$ определяется условием несгущаемости: $n(t+1)-n(t) \leqslant l$. Определим числа $s_{m}:=n(m+0), m \in \mathbb{Z}$. Тогда сегменты $\Delta_{m}=(m-1, m], m=1,2, \ldots$, содержат $l_{m}=s_{m}-s_{m-1} \leqslant l$ собственных значений из последовательности $\left\{\mu_{k}\right\}$. Теперь определим функцию $\psi(t)$ на интервалах $\Delta_{m+1}=(m, m+1]$ следующим образом:

$$
\psi(t)=s_{m}+l_{m}(t-m), \quad t \in(m, m+1] .
$$

Из построения следует, что $|\psi(t)-n(t)| \leqslant \sup \left\{l_{m}\right\}=l$ и $\psi^{\prime}(t) \leqslant l$. Тем самым представление (7.10) доказано.

Пользуясь этим представлением и интегрируя по частям правую часть равенства (7.9), получаем

$$
S(\lambda) x=\int_{\mathbb{R}} \frac{r(t) \psi^{\prime}(t)-r^{\prime}(t) \eta(t)}{t-\lambda} d t+\int_{\mathbb{R}} \frac{r(t) \eta(t)}{(t-\lambda)^{2}} d t=: S_{1}(\lambda) x+S_{2}(\lambda) x
$$

Поскольку $r(t) \in L_{2}(\mathbb{R})$ и $|\eta(t)| \leqslant l$, то функцию $S_{2}(\lambda) x$ на прямых $\Gamma_{ \pm}$можно оценить, воспользовавшись неравенством Коши-Буняковского, что влечет включение $S_{2}(\lambda) x \in L_{2}(\mathbb{R}+i h)$. Далее, $\left|\chi^{\prime}(t)\right| \leqslant 1$, поэтому $r^{\prime}(t) \in L_{2}(\mathbb{R})$. Но тогда

$$
f(t):=r(t) \psi^{\prime}(t)-r^{\prime}(t) \eta(t) \in L_{2}(\mathbb{R})
$$

Таким образом, функция $S_{1}(\lambda) x$ является преобразованием Гильберта функции $f(t) \in L_{2}(\mathbb{R})$. Но тогда $S_{1}(\lambda) x$ принадлежит $L_{2}(\mathbb{R}+i h)$ при любом $h \in \mathbb{R}$. Этот факт известен специалистам, его можно считать фольклором, но мы затрудняемся дать прямую литературную ссылку. Что эти утверждения справедливы, легко понять, прочитав главы 1-3 книги [75] о связи оператора Пуассона, оператора гармонического сопряжения и преобразования Гильберта (см. [75; теорема 2.5.6, следствия 2.4.3 и 3.3.4]). Отметим еще, что результаты этой теории излагаются для скалярных функций, но при их трактовке для вектор-функций никаких проблем не возникает. Достаточно разложить вектор-функцию по ортонормированному базису и работать с каждой координатой. Теперь, учитывая, что $S_{2}(\lambda) x \in L_{2}(\mathbb{R} \pm i h)$ при любом $h \neq 0$, получаем, что функция $S(\lambda) x$ обладает тем же свойством. 
Шаг 5. Докажем ограниченность оператор-функции $M(\lambda)$ вне исключительного множества кружков $\mathscr{K}_{A}$ с центрами в собственных значениях $\lambda_{k}$ оператора $A$. Образуем последовательность $\zeta_{k}=\lambda_{k}+i h$, выбрав $h$ так, чтобы эта последовательность лежала в открытой верхней полуплоскости. Построим по этой последовательности произведение Бляшке

$$
\mathscr{B}(\lambda)=\prod_{-\infty}^{\infty} \frac{\lambda-\zeta_{k}}{\lambda-\overline{\zeta_{k}}} \frac{\overline{\zeta_{k}}}{-\zeta_{k}} .
$$

Согласно представлению (7.6) функция $M(\lambda)$ имеет полюсы только в точках $\lambda_{k}$. Тогда функция

$$
\Phi(\lambda)=\mathscr{B}(\lambda) M(\lambda+i h)
$$

является голоморфной в верхней полуплоскости. Кроме того, $\|\Phi(\lambda)\| \leqslant 2$ на вещественной прямой и в полуплоскости $\operatorname{Im} \lambda \geqslant 2 h$, так как $\|M(\lambda)\| \leqslant 2$ в этой полуплоскости и $|\mathscr{B}(\lambda)| \leqslant 1$ в $\mathbb{C}_{+}$. Согласно теореме 4.2 порядок оператор-функции $(A-\lambda)^{-1}$ равен 1 , так как $A$ и $T$ имеют одинаковые порядки. Тогда по теореме Фрагмена-Линделёфа получаем неравенство $\|\Phi(\lambda)\| \leqslant 2$ во всей верхней полуплоскости.

Докажем следующее простое утверждение. Пусть $\zeta_{k}-$ несгущающаяся последовательность, $0<\operatorname{Im} \zeta_{k}<h$ u $\mathscr{K}$ - множество кружков радиуса є с иентрами в точках $\zeta_{k}$. Тогда произведение Бляшке, построенное по этой последовательности, допускает в полосе $0 \leqslant \operatorname{Im} \lambda \leqslant 2 h$ вне множества $\mathscr{K}$ следующую оченку снизу:

$$
|\mathscr{B}(\lambda)| \geqslant c=c(\varepsilon), \quad \operatorname{Im} \lambda \geqslant 0, \quad \lambda \notin \mathscr{K} .
$$

Действительно, если $\lambda=\sigma+i \tau$ и $\lambda_{k}=\sigma_{k}+i \tau_{k}$, то

$$
|\mathscr{B}(\lambda)|^{-2}=\prod_{k} \frac{\left(\sigma+\sigma_{k}\right)^{2}+\left(\tau-\tau_{k}\right)^{2}}{\left|\lambda-\zeta_{k}\right|^{2}}=\prod_{k}\left(1+\frac{4 \tau \tau_{k}}{\left|\lambda-\zeta_{k}\right|^{2}}\right) .
$$

Последовательность $\zeta_{k}$ назовем $\delta$-отделенной, если $\left|\zeta_{k+1}-\zeta_{k}\right|>\delta$. Ясно, что всякую несгущающуюся последовательность можно разбить на конечное число 1-отделенных последовательностей. Если $\left\{\zeta_{k}\right\}$ есть 1-отделенная последовательность, а $\mathscr{K}=\mathscr{K}(\varepsilon)-$ множество кружков радиуса $\varepsilon$ с центрами в $\zeta_{k}$, то найдется индекс $j$ такой, что $\left|\lambda-\zeta_{k}\right| \geqslant \varepsilon+|k-j| / 2$, если $\varepsilon \in(0,1 / 2)$. Тогда

$$
-2 \ln |\mathscr{B}(\lambda)| \leqslant \prod_{k}\left(1+\frac{16 h^{2}}{(\varepsilon+|k-j|)^{2}}\right) \leqslant C_{1}(\varepsilon), \quad 0 \leqslant \operatorname{Im} \lambda \leqslant 2 h .
$$

Поэтому $|\mathscr{B}(\lambda)| \geqslant c, c=\exp \left(-2 C_{1}(\varepsilon)\right)$. Для несгущающейся последовательности под экспоненту надо внести еще число $l$.

Ранее мы доказали, что $\|\Phi(\lambda)\| \leqslant 2$ в верхней полуплоскости. Но тогда из этого неравенства получаем

$$
\|M(\lambda)\| \leqslant 2 c^{-1}, \quad \operatorname{Im} \lambda \geqslant-h, \quad \lambda \notin \mathscr{K} .
$$


Шаг 6. Теперь рассмотрим голоморфную в верхней полуплоскости вектор-функцию

$$
\Psi(\lambda)=\mathscr{B}(\lambda) S(\lambda+i h) x,
$$

где $\mathscr{B}(\lambda)$ - произведение Бляшке, построенное по несгущающейся последовательности $\left\{\mu_{k}+i h\right\}$. Очевидно, что $\|\Psi(\lambda)\| \leqslant 1 / 2$ на вещественной оси и в полуплоскости $\operatorname{Im} \lambda \geqslant 2 h$. Согласно теореме Фрагмена-Линделёфа неравенство сохраняется во всей верхней полуплоскости, т. е. $\Psi$ принадлежит классу Харди $H^{\infty}\left(\mathbb{C}_{+}\right)$. Поскольку $|\mathscr{B}(\lambda)|=1$ при $\lambda \in \mathbb{R}$, то из доказанного ранее получаем

$$
\Psi(\sigma+i 0)=\Psi(\sigma) \in L_{2}(\mathbb{R}) .
$$

Таким образом, $\Psi \in H^{\infty}\left(\mathbb{C}_{+}\right)$, а граничные значения функции $\Psi$ принадлежат $L_{2}(\mathbb{R})$. Но тогда (см. [75; гл. 2]) функция $\Psi$ принадлежит классу Харди $H^{2}\left(\mathbb{C}_{+}\right)$. Из определения нормы для функций этого класса имеем

$$
\int_{-\infty}^{\infty} \int_{0}^{2 h}\|\Psi(\sigma+i \tau)\|^{2} d \tau d \sigma<C
$$

Пусть $\mathscr{K}=\mathscr{K}_{T} \cup \mathscr{K}_{A}$ - объединение кружков радиуса $\varepsilon$ с центрами в точках $\lambda_{k}$ и $\mu_{k}$. Если $\varepsilon$ достаточно мало, то на каждом отрезке $[k, k+1]$ можно найти отрезок $\Delta_{k}=\left[r_{k}, r_{k}+\varepsilon\right]$ такой, что в полосе $r_{k} \leqslant \operatorname{Re} \lambda \leqslant r_{k}+\varepsilon$ нет точек множества $\mathscr{K}$. Из неравенства (7.11) тогда получаем

$$
\sum_{k} \int_{\Delta_{k}} \int_{0}^{2 h}|\Psi(\sigma+i \tau)|^{2} d \tau d \sigma<C
$$

По теореме о среднем найдутся точки $\xi_{k} \in \Delta_{k}$ такие, что

$$
\int_{\Delta_{k}} \int_{0}^{2 h}|\Psi(\sigma+i \tau)|^{2} d \tau d \sigma=\varepsilon \int_{0}^{2 h}\left|\Psi\left(\xi_{k}+i \tau\right)\right|^{2} d \tau=: \varepsilon c_{k}^{2} .
$$

Из (7.11) следует, что $\varepsilon \sum c_{k}^{2}<C$. Обозначим через $\gamma_{k}$ вертикальные отрезки в полосе $|\operatorname{Im} \lambda| \leqslant h$, проходящие через точки $\xi_{k}$, и заметим, что на отрезках $\gamma_{k}+i h$ выполняется оценка $|\mathscr{B}(\lambda)|>c$. Тогда

$$
\begin{aligned}
\int_{\gamma_{k}}\|S(\lambda) x\|^{2}|d \lambda| & =\int_{\gamma_{k}+i h}\left\|\mathscr{B}^{-1}(\lambda) \Psi(\lambda-i h)\right\|^{2}|d \lambda| \\
& \leqslant c^{-1} \int_{0}^{2 h}\left\|\Psi\left(\xi_{k}+i \tau\right)\right\|^{2} d \tau=\varepsilon^{-1} c^{-1} c_{k}^{2}, \quad \text { где } \quad \sum c_{k}^{2}<\infty .
\end{aligned}
$$

Тем самым сходимость интегралов и рядов в (7.7) доказана, чем завершается доказательство теоремы 7.1 .

Естествен вопрос: можно ли ослабить условие регулярности поведения собственных значений $\mu_{k}$ невозмущенного оператора? Можно ли условие несгущаемости или условие (7.15), которое является обременительным в приложениях к дифференциальным операторам с частными производными, заменить 
на "необременительное" условие (7.3)? Автор склоняется к мнению, что ответ на этот вопрос отрицательный. Однако если вместо вопроса о безусловной базисности ставить вопрос о базисности обычной, ответ будет положительным.

Теорема 7.2. Пусть оператор $T=T^{*}$ uмеет порядок $\alpha$ и оператор $B$ локально р-подчинен оператору $T$ при некотором $p \in[0,1)$. Если выполнено условие $\alpha^{-1} \leqslant 1-p$, то корневые векторы оператора $T+B$ образуют базис со скобками в пространстве $H$.

Эта теорема проще, нежели предыдущая. Доказательство опускаем, предполагая провести его в отдельной работе.

Для рассмотренных в разделе 3 возмущений порядка $p$ спектр возмущенного оператора локализуется в параболах того же порядка $p$. Сохраняется ли это свойство при $p$-возмущениях локального типа? Ответ на этот вопрос положителен при выполнении условия несгущаемости, но порядок параболы может увеличиться. При этом мы допускаем, что порядок подчинения $p$ в условии (7.1) может быть и отрицательным. Сформулируем точный результат о локализации спектра.

ПРЕДЛОЖЕНИЕ 7.3. Пусть оператор $T=T^{*}$ uмеет порядок $\alpha$ и оператор $B$ локально р-подчинен оператору $T$ при некотором $-\infty<p<1$. Рассмотрим двойную параболическую область

$$
\Pi_{\nu, h}^{ \pm}=\left\{\lambda \in \mathbb{C}:|\operatorname{Re} \lambda| \leqslant h|\operatorname{Im} \lambda|^{\nu}\right\} .
$$

Пусть выполнено условие

$$
\gamma:=\max \left(0, p, 2 p+\alpha^{-1}-1\right)<1
$$

Если последовательность $\left\{\mu_{k}\right\}$ является $\alpha$-несгущающейся, то спектр оператора $A=T+B$ при достаточно большом $h$ асимптотически локализован в области $\Pi_{\gamma, h}^{ \pm}$. Вне этой области справедлива оценка

$$
\left\|(A-\lambda)^{-1}\right\| \leqslant C \tau^{-1}, \quad \lambda=\eta+i \tau
$$

Если оператор Т полуограничен, то спектр локализуется только внутри правой параболь.

Доказательство этого предложения для положительного оператора $T$ имеется в работе автора [52]. Однако оно с очевидными изменениями сохраняется для случая $T=T^{*}$ и даже для нормального оператора со спектром на конечном числе лучей, если область локализации определять как объединения параболических областей, примыкающих к лучам.

Докажем теорему о базисности для суммирования методом Абеля-Лидского.

ТЕОРема 7.4. Пусть T - самосопряженный полуограниченный снизу оператор порядка а и оператор В является локально р-подчиненным оператору $T$ при некотором $-\infty<p<1$. Предположим, что выполнено условие (7.13). Тогда система корневых векторов оператора $A=T+B$ образует базис для суммирования методом Абеля-Лидского порядка $\beta>\alpha^{-1}+\gamma-1$. 
ДокАзАтельство. Согласно предложению 7.3 спектр возмущенного оператора лежит в параболе порядка $\gamma$, а вне этой области выполнено соотношение (7.14). Теперь достаточно воспользоваться замечанием 5.4.

Приведем теперь некоторые результаты для локально $p$-подчиненных возмущений в смысле форм.

ПРЕДЛОЖеНИЕ 7.5. Пусть оператор $T$ имеет порядок $\alpha$ и форма $\mathfrak{b}=\mathfrak{b}[x, x]$ локально $p$-подчинена оператору $T$ при некотором $p<1$, причем $p<1-\alpha^{-1}$. Тогда форма $\mathfrak{b}$ является вполне Т-подчиненной (в смысле определения в разделе 2). В частности, форме $(T x, x)+\mathfrak{b}[x, x]$ соответствует оператор $T+B$, который замкнут в $Н$ и имеет дискретный спектр. Система корневых векторов оператора $T+B$ полна в $H$. Для всех $\lambda \notin \sigma(T+B) \cup \sigma(T)$ справедливо представление

$$
(T+B-\lambda)^{-1}=V(\lambda)(1+B(\lambda))^{-1} V(\lambda)
$$

¿əe

$$
V(\lambda)=(T-\lambda)^{-1}, \quad B(\lambda)=U(\lambda) B_{1}(\lambda), \quad U(\lambda)=V(\lambda)\left[V^{-1}\right]^{*},
$$

a оператор $B_{1}(\lambda)$ порождается ограниченной квадратичной формой

$$
\widehat{\mathfrak{b}}_{\lambda}[x, x]=\mathfrak{b}[V(\lambda) x, V(\lambda) x], \quad\|B(\lambda)\|=\left\|B_{1}(\lambda)\right\| \leqslant 2|\widehat{\mathfrak{b}}[x, x]|\|x\|^{-2} .
$$

ДоказАтельство. Обозначим $x_{k}=\left(x, \varphi_{k}\right)$. Тогда с учетом $(7.2)$ получаем

$$
\begin{aligned}
|\mathfrak{b}[V(\lambda) x, V(\lambda) x]| & =\left|\mathfrak{b}\left[\sum_{k} \frac{x_{k} \varphi_{k}}{\left(\mu_{k}-\lambda\right)^{1 / 2}}, \sum_{j} \frac{x_{j} \varphi_{j}}{\left(\mu_{j}-\lambda\right)^{1 / 2}}\right]\right| \\
& \leqslant\left|\sum_{k} \sum_{j} \frac{x_{k} x_{j} \mathfrak{b}\left[\varphi_{k}, \varphi_{j}\right]}{\left(\mu_{k}-\lambda\right)^{1 / 2}\left(\mu_{j}-\lambda\right)^{1 / 2}}\right| \\
& \leqslant b \sum_{k} \sum_{j} \frac{\left|x_{k}\right|\left|x_{j}\right|\left|\mu_{k}\right|^{p / 2}\left|\mu_{j}\right|^{p / 2}}{\left|\mu_{k}-\lambda\right|^{1 / 2}\left|\mu_{j}-\lambda\right|^{1 / 2}} \\
& \leqslant b\left(\sum_{k} \frac{\left|x_{k}\right|\left|\mu_{k}\right|^{p / 2}}{\left|\mu_{k}-\lambda\right|^{1 / 2}}\right)^{2} \leqslant b\left(\sum_{k} \frac{\left|\mu_{k}\right|^{p}}{\left|\mu_{k}-\lambda\right|}\right)\|x\|^{2} .
\end{aligned}
$$

Здесь суммирование ведется по всем индексам от $-\infty$ до $\infty$. Порядок оператора равен $\alpha$, поэтому $\left|\mu_{k}\right| \geqslant c|k|^{\alpha}$. По условию $p<1-\alpha^{-1}$, следовательно, ряд

$$
\sum\left|\mu_{k}\right|^{p-1} \leqslant \sum|k|^{\alpha(p-1)}
$$

сходится и форме $\widehat{\mathfrak{b}}_{\lambda}$ отвечает ограниченный оператор. При $\lambda=0$ получаем, что форма $\mathfrak{b}_{0}$ является $T$-подчиненной в смысле определения в разделе 2 . Пусть $P_{n}$ - ортопроекторы на линейную оболочку системы $\left\{\varphi_{k}\right\}_{k=-n}^{n}$. Тогда форма

$$
\widehat{\mathfrak{b}}_{0, n}=\mathfrak{b}\left[V(0) P_{n} x, V(0) P_{n} x\right]
$$

определяет конечномерные операторы, причем

$$
\left|\widehat{\mathfrak{b}}_{0}[x, x]-\widehat{\mathfrak{b}}_{0, n}[x, x]\right| \leqslant\|x\|^{2} \sum_{|k|>n}\left|\mu_{k}\right|^{p-1} \rightarrow 0 \quad \text { при } n \rightarrow \infty .
$$


В силу предложения 2.2 форма $\mathfrak{b}$ является вполне $T$-подчиненной, а тогда справедлива теорема 2.5. Оператор $(T+B-\lambda)^{-1}$ компактен, поскольку $V(\lambda)$ компактный. Поэтому спектр оператора $T+B$ дискретен. В силу следствия 4.11 система его корневых векторов полна в $H$. Представление для резольвенты $T+B$ следует из факторизации (2.12). Предложение доказано.

ТеОРема 7.6. ${ }^{2}$ Пусть выполнены условия предложения 7.5. Кроме того, пусть выполнено одно из условий

(i) $\alpha \geqslant 1 u\left\{\mu_{k}\right\}$ является $\alpha$-несгущающейся последовательностью;

(ii) выполнены соотношения $\mu_{k} \asymp k^{\alpha}$ и существуют числа $l \in \mathbb{N} u \tau>0$ такие, чmо

$$
\left|\mu_{k+l}-\mu_{k}\right| \geqslant \tau|k|^{\alpha-1}
$$

Обозначим через $\mathscr{K}_{\delta}$ объединение кружков

$$
\mathscr{K}_{j, \delta}=\left\{\lambda \in \mathbb{C}:\left|\lambda-\mu_{j}\right|<\delta j^{\alpha-1}\right\}, \quad j \in \mathbb{Z} .
$$

В случае (i) вместо кружков радиуса $\delta i^{\alpha-1}$ будем рассматривать кружки радиуса $\delta|\mu|^{1-\beta}$. Тогда при достаточно малом $\delta>0$ множество $\mathscr{K}_{\delta}$ разбивается на непересекаюшиеся компоненты $\Omega_{s}, s \in \mathbb{Z},|s| \geqslant s_{0}$, каждая из которых содержит $\leqslant l$ собственных значений обоих операторов $T$ и $T+B$, и существует ограниченная область $\Omega_{0}$, которая содержит все оставшиеся собственные значения операторов $T$ и $T+B$, не входящие в области $\left\{\Omega_{s}\right\}_{|s| \geqslant s_{0}}$. При этом проекторы Рисса $Q_{0}$ u $\left\{Q_{s}\right\}_{|s| \geqslant s_{0}}$ на части спектра оператора $T+B$ в обласmяx $\Omega_{0} u\left\{\Omega_{\mid} \mid\right\}_{s \geqslant s_{0}}$ образуют базис Бари, квадратично близкий $к$ системе соответствуюших проекторов $P_{0} u\left\{P_{s}\right\}_{|s| \geqslant s_{0}}$ оператора $T$.

ДокАЗАТЕЛЬСтво. При выполнении условия (ii) утверждение о базисности Рисса (вместо базисности Бари) в случае $T>0$ и $l=1$ доказано в недавней работе Митягина и Зигла [76]. Этой работе предшествовала их работа [50], где рассматривался случай $\alpha=1$. Для доказательства в [76] использовалась лемма Като (предложение 6.2), мы же используем теорему Бари-Маркуса в уточненной форме (теорема 6.3). Переход от случая $T>0$ к случаю $T=T^{*}$ не усложняет существенно проведение оценок, но требует новую интерпретацию суммы $T+B$, основанную на теореме 2.5 и предложении 7.5. Аргументы, приведенные в [76] для доказательства в случае $l=1$ (в этом случае проекторы асимптотически одномерные), не переносятся на случай $l>1$. Наш метод доказательства проще, кроме того, при его использовании значение числа $l$ по существу роли не играет.

Приведем доказательство, опуская несущественные детали.

Предположим, что выполнено условие (ii). Пусть сначала для простоты $l=1$ и $\mu_{k}>0$. Зафиксируем число $\delta \in(0, \tau / 2)$. Из условия (7.15) следует, что кружки $\mathscr{K}_{j, \delta}$ асимптотически не пересекаются. Если $\lambda$ лежит на границе

\footnotetext{
${ }^{2}$ В первоначальном варианте этой статьи имелась только формулировка теоремы 7.6, причем условие (ii) не фигурировало. Исправленный вариант с доказательством теоремы 7.6 был представлен в редакцию 25 августа 2016 г. после опубликования статьи Б. С. Митягина и П. Зигла [76].
} 
кружка $\mathscr{K}_{j, \delta}$, то

$$
\begin{aligned}
& \left|\mu_{k}-\lambda\right|=\delta j^{\alpha-1} \quad \text { при } k=j ; \\
& \left|\mu_{k}-\lambda\right| \geqslant\left|\mu_{k}-\mu_{j}\right|-\delta j^{\alpha-1} \geqslant \frac{\tau}{2}\left|\mu_{k}-\mu_{j}\right| \geqslant \tau_{1}\left|j^{\alpha}-k^{\alpha}\right| \quad \text { при } k \neq j,
\end{aligned}
$$

где $\tau_{1}=$ const. Последняя оценка вытекает из (7.15) (см. подробнее [76; лемма 4.1]. Поэтому при $\lambda \in \partial \mathscr{K}_{j, \delta}$

$$
\sum_{k=1}^{\infty} \frac{\left|\mu_{k}\right|^{p}}{\left|\mu_{k}-\lambda\right|} \leqslant C \frac{j^{\alpha p}}{\delta j^{\alpha-1}}+C\left(\sum_{k=1}^{j-1}+\sum_{k=j+1}^{\infty}\right) \frac{k^{\alpha p}}{\tau_{1}\left|j^{\alpha}-k^{\alpha}\right|} .
$$

Сумма в правой части оценивается точно так же, как на шаге 4 в доказательстве теоремы 6.6. Более того, в силу условия $\alpha-1-\alpha p=\alpha\left(1-p-\alpha^{-1}\right)>0$ правая часть стремится к нулю при $j \rightarrow \infty$.

Если $l>1$, то оценки сохранятся. В этом случае число $\delta$ надо подчинить условию $\delta \in(0,1 /(2 l))$ и учесть, что тогда множество $\mathscr{K}_{\delta}$ разобьется на непересекающиеся компоненты $\Omega_{s}$, каждая из которых асимптотически представляет собой объединение не более чем $l$ кружков $\mathscr{K}_{j, \delta}$. Оценка $(7.17)$ остается справедливой при $\lambda \in \Gamma_{s}:=\partial \Omega_{s}$, только правую часть нужно умножить на $l$. Если последовательность $\left\{\mu_{k}\right\}_{k=0}^{-\infty}$ бесконечна, то оценки также сохраняются. Можно рассуждать так. Добавим к последовательности $\left\{\mu_{k}\right\}_{k=1}^{\infty}$ последовательность $\left\{\left|\mu_{k}\right|\right\}_{k=-1}^{-\infty}$. Очевидно, что при $k \leqslant 0$ имеем

$$
\left|\mu_{j}-\mu_{k}\right| \geqslant\left|\mu_{j}-\right| \mu_{k}||
$$

(считаем для определенности, что $j>0$ ). Поэтому добавление к сумме (7.17) аналогичной суммы по неположительным индексам не оказывает влияния на общую оценку. Полученная оценка (7.17) показывает, что, если $\lambda$ принадлежит $\Gamma_{j}$ - границе области $\Omega_{j}=\Omega_{j, \delta}$, то $\|B(\lambda)\| \rightarrow 0$ при $j \rightarrow \pm \infty$. Стандартным путем (рассматривая семейство операторов $T+t B$ при $0 \leqslant t \leqslant 1$ и используя факторизацию резольвенты в предложении 7.5) получаем, что число собственных значений операторов $T$ и $T+B$ в областях $\Omega_{s}$ при достаточно больших $|s| \geqslant s_{0}$ совпадает, а потому не превышает $l$.

Из приведенных оценок видно, что на прямых $\gamma_{j}$, разделяющих области $\Omega_{j}$ и $\Omega_{j+1}$, выполняется оценка $\|B(\lambda)\|<1 / 2$, если $|s| \geqslant s_{0}$. Такая же оценка выполняется на горизонтальных прямых $\operatorname{Im} \lambda= \pm h$, если число $h$ достаточно велико, а также вне полосы, ограниченной этими прямыми. Пусть $\Omega_{0}-$ прямоугольник, заключенный между прямыми $\operatorname{Im} \lambda= \pm h$ и вертикальными прямыми $\gamma_{ \pm s_{0}}$. Несложно показать, что вне областей $\left\{\Omega_{s}\right\}|s| \geqslant s_{0}$ и $\Omega_{0}$ нет спектра обоих операторов $T$ и $T+B$. Оценим разность проекторов (сдвигаем индексы, считая, что $\left.s_{0}=1\right)$ :

$$
\begin{aligned}
\sum_{s} \| & \left(Q_{s}-P_{s}\right) x\left\|^{2}=\sum_{s}\right\| \int_{\Gamma_{s}}\left((T+B-\lambda)^{-1}-(T-\lambda)^{-1}\right) x d \lambda \|^{2} \\
& \leqslant\left(\int_{\Gamma_{s}}\left\|(T-\lambda)^{-1 / 2}\right\|\left\|(1+B(\lambda))^{-1}\right\|\left\|B(\lambda)(T-\lambda)^{-1 / 2} x\right\||d \lambda|\right)^{2} \\
& \leqslant C \sum_{s}|s|^{\alpha-1}\left(\sup _{\lambda \in \Gamma_{s}}\left\|B(\lambda)(T-\lambda)^{-1} x\right\|\right)^{2} .
\end{aligned}
$$


Здесь мы учли, что при $\lambda \in \Gamma_{s}$ выполняется оценка $\left\|(1+B(\lambda))^{-1}\right\|<2$, a $\left\|(T-\lambda)^{-1 / 2}\right\|$ после умножения на длину контура интегрирования оценивается величиной $C^{1 / 2}|s|^{(\alpha-1) / 2}$. Далее, при $\lambda \in \Gamma_{s}$ получаем (полагаем $x=\sum_{j} x_{j} \varphi_{j}$, $y=\sum_{k} y_{k} \varphi_{k}$ и используем оценки $\left.(7.16)\right)$ :

$$
\begin{aligned}
\left\|B(\lambda)(T-\lambda)^{-1 / 2} x\right\|^{2} & =\sup _{\|y\|=1}\left|\left(B(\lambda)(T-\lambda)^{-1 / 2} x, y\right)\right|^{2} \\
& =\sup _{\|y\|=1}\left|\sum_{k} \sum_{j} \frac{\mathfrak{b}\left[\varphi_{j}, \varphi_{k}\right] x_{j} y_{k}}{\left(\mu_{j}-\lambda\right)\left(\mu_{k}-\lambda\right)^{1 / 2}}\right|^{2} \\
& \leqslant b^{2}\left(\sum_{k} \frac{\left|\mu_{k}\right|^{p}}{\left|\mu_{k}-\lambda\right|}\right)\left(\sum_{j} \frac{\left|\mu_{j}\right|^{p / 2}\left|x_{j}\right|}{\left|\mu_{j}-\lambda\right|}\right)^{2} \\
& \leqslant C\left(\sum_{k \neq s} \frac{k^{\alpha p}}{\left|k^{\alpha}-s^{\alpha}\right|}+s^{-\varepsilon}\right)\left(\sum_{j=\neq s} \frac{j^{\alpha p / 2}\left|x_{j}\right|}{\left|j^{\alpha}-s^{\alpha}\right|}+s^{-\varepsilon_{1}}\left|x_{s}\right|\right)^{2} .
\end{aligned}
$$

В этих оценках при $j<0$ мы полагаем $j^{\alpha}:=-|j|^{\alpha}$ и вводим обозначения

$$
\varepsilon:=(\alpha-1)-\alpha p=\alpha\left(1-p-\alpha^{-1}\right), \quad \varepsilon_{1}=(\alpha-1)-\frac{\alpha p}{2} .
$$

По условию теоремы $p<1-\alpha^{-1}$, поэтому $\varepsilon>0$. Нетрудно видеть (см. лемму 4.3 в работе [76]), что

$$
\sum_{k \neq s} \frac{1}{k^{-\alpha p}\left|k^{\alpha}-s^{\alpha}\right|} \leqslant C \max \left(|s|^{-\varepsilon} \ln |s|,|s|^{-\alpha}\right) .
$$

Можно считать, что $\varepsilon<\alpha$. Иначе можно увеличить число $p$ (оценка (7.2) при увеличении числа $p$ сохраняется). Из приведенных оценок получаем

$$
\sum_{s}\left\|\left(Q_{s}-P_{s}\right) x\right\|^{2} \leqslant C_{1}\left(\sum_{k \neq s} \frac{|j s|^{(\alpha-1) / 2-\varepsilon / 2}(\ln |s|)^{1 / 2} \mid}{\left|j^{\alpha}-s^{\alpha}\right|}\left|x_{j}\right|+s^{-\varepsilon / 2}(\ln |s|)^{1 / 2}\left|x_{s}\right|\right)^{2} .
$$

Здесь мы учли, что $\alpha p / 2=(\alpha-1) / 2-\varepsilon / 2,-\varepsilon_{1}+(\alpha-1) / 2=-\varepsilon / 2$. Поэтому проекторы квадратично близки, если матрица с элементами

$$
\frac{|j s|^{(\alpha-1) / 2-\varepsilon / 2}(\ln |s|)^{1 / 2}}{\left|j^{\alpha}-s^{\alpha}\right|}, \quad j \neq s,
$$

порождает в двустороннем пространстве $l_{2}$ ограниченный оператор. Но это так, поскольку при любом $\varepsilon>0$ к этой матрице примени́м тест Шура (см. [77; теорема 301]. Остается воспользоваться теоремой 6.3 и учесть, что в силу предложения 7.5 система проекторов $\left\{Q_{s}\right\}$ полна в пространстве $H$.

Пусть теперь выполнено условие (i) теоремы. При этом условии последовательность $\left\{\mu_{k}^{\beta}\right\}$ несгущающаяся, поэтому ее можно разбить на конечное число последовательностей, для которых выполнено условие $\left|\mu_{k+1}^{\beta}-\mu_{k}^{\beta}\right| \geqslant 1$. Приведенные выше аргументы позволяют свести доказательство к случаю, когда 
имеется только одна такая последовательность. Вместо кружков $\mathscr{K}_{j, \delta}$ радиуса $\delta j^{\alpha-1}$ мы рассматриваем кружки радиуса $\delta\left|\mu_{j}\right|^{1-\beta}$. Если $\beta \leqslant 1$, то можно воспользоваться оценками (6.29), которые вытекают из условия несгущаемости последовательности $\left\{\mu_{k}^{\beta}\right\}$. Тогда

$\left|\mu_{k}-\mu_{s}\right| \geqslant \max \left(\left|\mu_{k}\right|^{1-\beta},\left|\mu_{s}\right|^{1-\beta}\right)|k-s|, \quad\left|\mu_{k}\right| \geqslant c|k|^{\alpha}, \quad \beta=\alpha^{-1}, \quad c=$ const.

Учитывая эти оценки и пользуясь проведенными выше неравенствами, задачу о квадратичной близости проекторов сведем к задаче об ограниченности оператора, порожденного в двустороннем пространстве $l_{2}$ матрицей, элементы которой равны

$$
\frac{\left|\mu_{j} \mu_{s}\right|^{-\varepsilon / 2}(\ln |s|)^{1 / 2}}{|j-s|} \leqslant C \frac{|j s|^{-\varepsilon \alpha / 2}(\ln |s|)^{1 / 2}}{|j-s|}, \quad \varepsilon=1-p-\beta>0 .
$$

Тем самым, при выполнении условия (i) доказательство теоремы значительно упрощается.

Отметим также, что в случае $\alpha \leqslant 1$ (или $\beta \geqslant 1$ ) условие $\mu_{k} \asymp k^{\alpha}$ в (ii) можно опустить. В этом случае $p<0$, а тогда для доказательства достаточно выполнения неравенств $\mu_{k} \leqslant c k^{\alpha p}$, которые следуют из (7.15). Теорема доказана.

\section{8. Теоремы сравнения спектров}

Для самосопряженного оператора $T$ определим функции распределения его собственных значений $\left\{\mu_{k}\right\}$ :

$$
n_{T}^{+}(r)=\sum_{0 \leqslant \mu_{k}<r} 1, \quad n_{T}^{-}(r)=\sum_{-r \leqslant \mu_{k}<0} 1 .
$$

Функции распределения собственных значений $\left\{\lambda_{k}\right\}$ возмущенного оператора $A+B$ определим аналогично:

$$
n_{A}^{+}(r)=\sum_{0 \leqslant \operatorname{Re} \lambda_{k}<r} 1, \quad n_{A}^{-}(r)=\sum_{-r \leqslant \operatorname{Re} \lambda_{k}<0} 1 .
$$

Сформулируем несколько теорем сравнения этих функций.

Теорема 8.1. Пусть оператор $B$ является р-подчиненным оператору $T$ при некотором $p \in[0,1)$, a $b^{\prime}-$ точная грань р-подчиненности. Тогда при любом $b>b^{\prime}$ выполняется оценка

$$
\left|n_{T}^{ \pm}(r)-n_{A}^{ \pm}(r)\right| \leqslant C\left(n_{T}^{ \pm}\left(r+b r^{p}\right)-n_{T}^{ \pm}\left(r-b r^{p}\right)\right)+C_{0},
$$

где $C, C_{0}-$ постоянные, зависящие от $b$.

ТеОрема 8.2. Пусть оператор $B$ является р-подчиненным оператору $T$ в смысле квадратичнъх форм при некотором $p \in[0,1)$, a $b^{\prime}-$ точная грань $p$-подчиненности. Тогда при любом $b>2 b^{\prime}$ выполняется ощенка (8.2).

Теорема 8.3. Пусть оператор В является сильно р-подчиненным оператору $T$ при некотором $p \in(-\infty, 1)$. Тогда найдутся постоянные $b, C, C_{0}, n p и$ которых выполнена оценка (8.2). 
Tеорема 8.4. Пусть оператор В вполне T-подчинен или вполне T-подчинен в смысле форм. Тогда для любого $\varepsilon>0$ найдутся постоянные $C, C_{0}$, зависящие от $\varepsilon$, при которых выполнена оценка

$$
\left|n_{T}^{ \pm}(r)-n_{A}^{ \pm}(r)\right| \leqslant C\left(n_{T}^{ \pm}((1+\varepsilon) r)-n_{T}^{ \pm}((1-\varepsilon) r)\right)+C_{0} .
$$

Теорема 8.5. Пусть оператор В локально р-подчинен оператору $T$, а последовательности его положительных и отрицательных собственных значений являются $\alpha$-несгущающимися. Тогда найдутся постоянная $b$ и зависящие от нее постоянные $C, C_{0}$ такие, что

$$
\left|n_{T}^{ \pm}(r)-n_{A}^{ \pm}(r)\right| \leqslant C\left(n_{T}^{ \pm}\left(r+b r^{\gamma}\right)-n_{T}^{ \pm}\left(r-b r^{\gamma}\right)\right)+C_{0} .
$$

ЗАмЕчАниЕ 8.6. Если оператор $T$ положителен или полуограничен снизу, то оценки в теоремах 8.1-8.5 можно уточнить. В этом случае вместо функций $n_{T}^{+}$и $n_{A}^{+}$нужно рассмотреть функции, считающие все собственные значения, включая те, которые лежат в левой полуплоскости. Тогда во всех теоремах константу $C_{0}$ можно положить равной нулю.

В менее точной форме (без учета определений (3.2) и (3.6), т. е. без указания точной грани констант $b$ в оценке (8.3)) теоремы 8.1-8.4 получены А. С. Маркусом и В.И. Мацаевым [58], [54], [28]. Теорема 8.5 получена автором в [52] при $T>0$. Но ее доказательство сохраняется для случая $T=T^{*}$.

Здесь мы приведем только доказательство теоремы 8.1, для которого подготовительная работа уже проделана (остальные теоремы доказываются аналогично). Пусть сначала $T>0$. Зафиксируем число $r \gg 1$ и рассмотрим прямоугольник $P_{r}$, ограниченный горизонтальными прямыми $\operatorname{Im} \lambda= \pm h r^{p}$ и вертикальными прямыми $\operatorname{Re} \lambda=-r_{0}, \operatorname{Re} \lambda=r$, где $h$ и $r_{0}$ достаточно большие числа. Так же как на шаге 5 в доказательстве теоремы 6.6, рассмотрим конечномерный оператор $K_{r}$ вида (6.34) такой, что оператор $T_{r}=T-K_{r}$ сохраняет систему собственных функций оператора $T$, но меняет собственные значения

$$
\mu_{k} \in\left(r_{-}, r_{+}\right)=\left(r-b r^{p}, r+b r^{p}\right)
$$

сдвигая их в ближайший из концов $r_{+}$или $r_{-}$. Тогда

$$
\operatorname{rank} K_{r}=R(r):=n_{T}\left(r+b r^{p}\right)-n_{T}\left(r-b r^{p}\right), \quad\left\|K_{r}\right\| \leqslant R(r) .
$$

Справедливо представление

$$
1+K_{r}\left(T_{r}+B-\lambda\right)^{-1}=(A-\lambda)\left(T_{r}+B-\lambda\right)^{-1} .
$$

В силу формулы Вайнштейна-Ароншайна (см. [32; гл. 5]) имеем

$$
n_{A}(r)=n_{T_{r}+B}(r)+\nu(r),
$$

где $\nu(r)$ - разность между числом нулей и числом полюсов скалярной функции $D(\lambda)=\operatorname{det}\left(1-K_{r}\left(\lambda-T_{r}-B\right)^{-1}\right)$, лежащих в прямоугольнике $P_{r}$. При $\varepsilon \in[0,1]$ выполнено равенство

$$
\left(T_{r}+\varepsilon B-\lambda\right)^{-1}=\left(T_{r}-\lambda\right)^{-1}\left(1+\varepsilon S_{r}(\lambda)\right)^{-1}, \quad S_{r}(\lambda)=B\left(T_{r}-\lambda\right)^{-1} .
$$


Из приведенных в разделе 3 оценок резольвенты следует, что $\left\|S_{r}(\lambda)\right\|<q<1$ на левой и горизонтальных сторонах прямоугольника $P_{r}$. На правой стороне неравенство сохраняется, что было доказано на шаге 5 в теореме 6.6. Следовательно, проекторы Рисса

$$
Q_{\varepsilon}=-\frac{1}{2 \pi i} \int_{\partial P_{r}}\left(T_{r}+\varepsilon B-\lambda\right)^{-1} d \lambda=-\frac{1}{2 \pi i} \int_{\partial P_{r}}\left(T_{r}-\lambda\right)^{-1}\left(1+\varepsilon S_{r}(\lambda)\right)^{-1} d \lambda
$$

корректно определены и непрерывно зависят от $\varepsilon$ в равномерной операторной топологии. Согласно лемме Рисса-Сёкефальви-Надя [57; гл. 1, лемма 3.1]

$$
\operatorname{rank} Q_{\varepsilon_{1}}=\operatorname{rank} Q_{\varepsilon_{2}}
$$

если разность $\left|\varepsilon_{1}-\varepsilon_{2}\right|$ мала. Отсюда следует, что $\operatorname{rank} Q_{0}=\operatorname{rank} Q_{1}$, а тогда $n_{T_{r}+B}(r)=n_{T}(r)$. Из равенства (8.5) получаем

$$
n_{T_{r}+B}(r)=n_{T}(r) .
$$

Остается оценить $\nu(r)$ в формуле (8.5). Заметим, что аргумент функции $D(\lambda)$ почти не меняется на левой боковой и горизонтальных сторонах прямоугольника $P_{r}$ в силу малости $\left\|S_{r}(\lambda)\right\|$. А на правой боковой стороне мы оценили $D(\lambda)$ сверху и снизу числами $2^{R(r)}$ и $2^{-R(r)}$, где $R(r)$ определено в (8.4). Теперь остается воспользоваться предложением 6.5, из которого следует справедливость оценки (6.15).

Если оператор $T$ не полуограничен, то нужно ввести дополнительную искусственную лакуну в окрестности $\lambda=0$. А именно, вместо $K_{r}$ нужно ввести оператор $K_{r}+K_{0}$, где $K_{0}$ меняет собственные значения $\mu_{k} \in\left(-r_{0}, r_{0}\right)$, сдвигая их в концы этого интервала (мы предполагаем, что $r_{0}$ выбрано достаточно большим). Дальнейшие изменения очевидны.

Из приведенных теорем получаем важные приложения к дифференциальным операторам, имея в виду, что задача о формулах распределения собственных значений конкретных дифференциальных невозмущенных операторов хорошо изучена в литературе (см., например, [63]). Приведем одно из возможных следствий, доказательство которого очевидно.

СлеДСТВИЕ 8.7. Пусть $n_{T}^{+}(r)=r^{\beta}+O\left(r^{\eta}\right), \eta<\beta$. Если оператор А является р-подчиненным оператору $T$, то

$$
n_{A}^{+}(r)=r^{\beta}+O\left(r^{\eta}\right)+O\left(r^{\beta+p-1}\right)
$$

В случае очень слабых возмущений (при $p<0$ ) теоремы сравнения спектров в терминах считающих функций $n(t)$ дают грубые результаты. В этих случаях более точную информацию дают асимптотические формулы непосредственно для самих собственных значений $\lambda_{k}$ оператора $A=T+B$ или для разности $\lambda_{k}-\mu_{k}$. Очень интересные результаты об асимптотиках собственных значений и собственных функций операторов, возмущенных локально подчиненными формами, получены в недавних работах [40] и [76]. 


\section{9. Асимптотические методы. Примеры}

Асимптотические методы для доказательства сходимости спектральных разложений используются издавна. В этом разделе речь пойдет только об обыкновенных дифференциальных операторах. Работы на эту тему для операторов с частными производными автору не известны. Первые общие результаты о сходимости рядов по корневым функциям обыкновенных дифференциальных операторов на конечном интервале получил Дж. Биркгоф [66], [67]. Он выделил класс регулярных краевых условий и доказал теорему о сходимости разложений в ряды по корневым функциям регулярных операторов. Полезные обобщения результатов Биркгофа получил Я. Д. Тамаркин [4]. Во всех случаях результаты о сходимости разложений опирались на асимптотические формулы для решений резольвентного уравнения.

Интерес к задачам о базисности и безусловной базисности корневых векторов операторов возник в пятидесятые годы прошлого века. Стоит отметить, что операторы, собственные функции которых образуют базис Рисса, подобны нормальным. Для нормальных операторов имеется функциональное исчисление. Построение теории операторов, для которых можно строить функциональное исчисление, было предпринято в книге Н. Данфорда и Дж. Т. Шварца [53]. В качестве возможного приложения Данфорд [78] доказал теорему о безусловной базисности регулярного обыкновенного дифференциального оператора на конечном отрезке. В этой работе он не использовал методы теории возмущений и опирался на асимптотические формулы Биркгофа. Получив асимптотические формулы для собственных функций $\left\{\varphi_{k}\right\}$ и $\left\{\psi_{k}\right\}$ прямого и сопряженного операторов, он показал, что обе системы являются бесселевыми, т. е.

$$
\sum\left|\left(f, \varphi_{k}\right)\right|^{2}<\infty, \quad \sum\left|\left(f, \psi_{k}\right)\right|^{2}<\infty .
$$

Тогда в силу теоремы Бари обе системы являются базисами Рисса. Такой же результат для сильно регулярных операторов получили В. П. Михайлов [79] и Г. М. Кессельман [80], причем Кессельман построил пример, показывающий, что для регулярных, но не сильно регулярных операторов свойство базисности может нарушаться. Теорема Данфорда была "реабилитирована" в работе автоpa [81], где было показано, что свойство безусловной базисности сохраняется, если говорить о базисности двумерных подпространств, которые образуют собственные функции, отвечающие “слипающимся" собственным значениям. Но прежние методы работ [78]-[80] для доказательства этого замечания не проходили. В последующих работах [82], [83] были развиты другие, более общие методы доказательства безусловной сходимости спектральных разложений. Основой этих подходов были по-прежнему асимптотические формулы Биркгофа.

Для дифференциальных операторов с сингулярными коэффициентами формул Биркгофа в их обычном виде нет. Получить их аналоги и научиться ими пользоваться - нелегкая работа. В настоящее время она проведена только для некоторых конкретных операторов. В работе [84] получены асимптотические формулы для решений уравнения

$$
-y^{\prime \prime}+q(x) y=\rho^{2} y, \quad x \in[0,1], \quad q \in W_{2}^{-1}[0,1], \quad \rho^{2}=\lambda
$$


(здесь $W_{2}^{-1}[0,1]$ - пространство Соболева, состоящее из функций $f$, для которых первообразная $\int f$, понимаемая в смысле теории распределений, принадлежит $\left.L_{2}[0,1]\right)$. В недавней работе [85] получены асимптотические формулы для решений системы Дирака

$$
-T \mathbf{y}^{\prime}+Q(x) \mathbf{y}=\lambda \mathbf{y}, \quad T=\left(\begin{array}{cc}
0 & 1 \\
-1 & 0
\end{array}\right), \quad Q(x)=\left(\begin{array}{cc}
q_{1}(x) & q_{2}(x) \\
q_{3}(x) & q_{4}(x)
\end{array}\right),
$$

с потенциалом $Q \in L_{1}[0,1]$. Эти результаты были использованы для доказательства безусловной базисности корневых функций соответствующих операторов.

"Свежим ветром" явилась для автора работа Дж. Аддучи и Б. С. Митягина [41], где была доказана теорема о безусловной базисности возмущенного гармонического осциллятора $T+B$ :

$$
T y=-y^{\prime \prime}+x^{2} y, \quad B y=q(x) y, \quad \mathscr{D}(T)=W_{2}^{2}(\mathbb{R}) .
$$

Для доказательства этого результата авторы [41] работали не с асимптотиками решений соответствующего резольвентного уравнения $(T+B) y=\lambda y$, а с асимптотиками собственных функций невозмущенного гармонического осциллятора. Эти функции - многочлены Эрмита, их асимптотические свойства хорошо изучены. Продолжение исследований этой задачи было предпринято в работах [50], [76] и [40].

В заключение на примере нескольких простых модельных задач покажем, насколько эффективны для них разные подходы. Для простоты говорим только об условиях Дирихле. Потенциалы в примерах комплекснозначные. Обсуждаем только задачу о базисности.

Пример 1. Оператор Лапласа в двумерной ограниченной области $\Omega \subset \mathbb{R}^{2}$ : $T u=-\Delta u$ с краевыми условиями Дирихле, $B u=q u$.

Пример 2. Оператор Штурма-Лиувилля с потенциалом из $q \in W_{2}^{-1}[0,1]$ : $T y=-y^{\prime \prime}$ с краевыми условиями Дирихле, $B y=q y$.

ПримеР 3. Оператор Штурма-Лиувилля с возмущением первого порядка: $T y=-y^{\prime \prime}$ с краевыми условиями Дирихле, $B y=q y^{\prime}, q \in L_{2}[0,1]$.

Пример 4. Возмущение гармонического осциллятора: $T y=-y^{\prime \prime}+x^{2} y$ в пространстве $L_{2}(\mathbb{R}), B y=q y$.

Пример 5. Оператор Дирака $T+B$, где $B \mathbf{y}=Q(x) \mathbf{y}$, а оператор $T$ и матрица-функция $Q$ определены в (9.1).

Корневые функции оператора в примере 1 образуют базис Рисса со скобками, если $q \in L_{\infty}(\Omega)$. Это следует из теоремы 6.6 или теоремы 6.9. Здесь мы учитываем, что $\mu_{k}(T) \geqslant c k$. Для функций $q \in L_{2}(\Omega)$ эти теоремы не работают. Однако если собственные функции оператора $T$ подчинены оценкам

$$
\left\|\varphi_{k}\right\|_{L_{2}} \asymp\left\|\varphi_{k}\right\|_{L_{\infty}}
$$


то для $q \in L_{2}$ выполнено условие локальной 0-подчиненности, и тогда согласно теореме 7.2 собственные функции образуют базис. Теорему 7.1 для доказательства базисности Рисса использовать нельзя, так как последовательность собственных значений оператора $T$ имеет кластеры и не является несгущающейся. Оценки (9.2) заведомо выполнены, если область $\Omega$ - прямоугольник.

В примере 2 можно показать, что оператор $B$ вполне $T$-подчинен в смысле квадратичных форм (см. [84]), но $p$-подчиненности при $p<1$ нет. Поэтому результаты раздела 6 не применимы. Однако корневые функции этого оператора образуют базис Рисса, что доказано в [84] с помощью асимптотических методов.

В примере $3 \mu_{k} \asymp k^{2}$, выполнено условие локальной $1 / 2$-подчиненности и последовательность $\left\{\mu_{k}\right\}$ является 1/2-несгущающейся, поэтому применима теорема 7.1. Никакие другие подходы не работают.

Пример 4 был рассмотрен Дж. Аддучи и Б.С. Митягиным в работе [41]. Они доказали безусловную базисность в случае $q \in L_{2}(\mathbb{R})$. Для функций $q$ из $L_{2}(\mathbb{R})+L_{\infty}(\mathbb{R})$ их теорема не применима, работает только теорема 7.1. В работе [50] для этого примера получена безусловная базисность в случае потенциала $q=\sum c_{k} \delta\left(x-x_{k}\right)$, где $\sum\left|c_{k}\right|<\infty$; в более общей форме этот результат представлен в теореме 7.6. Для таких потенциалов теорема 7.1 не работает.

В примере 5 результаты раздела 6 можно применить только для $Q \in L_{\infty}$. Теорема 7.1 работает для потенциалов из $L_{2}$. Для таких же потенциалов этот результат ранее получен в [45]. Для потенциалов из $L_{p}$ при $p \in[1,2)$ ни теорема 7.1, ни метод [45] не работают. Однако с помощью асимптотических методов безусловная базисность для $Q \in L_{1}$ при любых регулярных краевых условиях получена в [85]. Развивая технику операторов преобразования, этот же результат независимо получили А. А. Лунёв и М. М. Маламуд [86], но только для сильно регулярных операторов. Подробные доказательства результатов работы [86] и их обобщений были приведены ими в работе [87].

В связи с оператором Дирака следует отметить недавние работы И. В. Садовничей [88], [89]. Из ее результатов, в частности, получается базисность (конечно, условная) корневых функций регулярного оператора Дирака в пространствах $L_{p}$ при $p \neq 2$.

Важные приложения и примеры изложенных здесь результатов для операторов с частными производными имеются в работах [58], [50] и [76].

\section{Список литературы}

[1] М. В. Келдыш, "О собственных значениях и собственных функциях некоторых классов несамосопряженных уравнений”, Докл. АН СССР, 77:1 (1951), 11-14.

[2] М.В. Келдыш, “Об одной тауберовой теореме”, Сборник статей. Посвящается академику Ивану Матвеевичу Виноградову к его 60-летию, Тр. МИАН СССР, 38, Изд-во АН СССР, М., 1951, 77-86; англ. пер.: M. V. Keldysh, "On a Tauberian theorem", Amer. Math. Soc. Transl. Ser. 2, 102, Amer. Math. Soc., Providence, RI, 1973, 133-143.

[3] М.В. Келдыш, "О полноте собственных функций некоторых классов несамосопряженных линейных операторов", УМН, 26:4(160) (1971), 15-41; англ. пер.: 
M.V. Keldysh, "On the completeness of the eigenfunctions of some classes of non-selfadjoint linear operators", Russian Math. Surveys, 26:4 (1971), 15-44.

[4] Я. Д. Тамаркин, О некоторых общих задачах теории обыкновенных линейных дифференииальных уравнений и о разложении произволъных функиий в ряды, Тип. М. П. Фроловой, Петроград, 1917, xiv+308 c.

[5] T. Carleman, "Über die asymptotische Verteilung der Eigenwerte partieller Differentialgleichungen", Ber. Verh. Sächs. Akad. Leipzig, 88 (1936), 119-132.

[6] F.E. Browder, "On the eigenfunctions and eigenvalues of the general linear elliptic differential operator", Proc. Nat. Acad. Sci. U. S. A., 39 (1953), 433-439.

[7] F.E. Browder, "On the spectral theory of strongly elliptic differential operators", Proc. Nat. Acad. Sci. U. S. A., 45 (1959), 1423-1431.

[8] F.E. Browder, "On the spectral theory of elliptic differential operators. I", Math. Ann., 142 (1961), 22-130.

[9] М. С. Лившиц, "О спектральном разложении линейных несамосопряженных операторов", Матем. сб., 34(76):1 (1954), 145-199; англ. пер.: M. S. Livshits, "On spectral decomposition of linear non-selfadjoint operators", Amer. Math. Soc. Transl. Ser. 2, 5, Amer. Math. Soc., Providence, RI, 1957, 67-114.

[10] Б. Р. Мукминов, "О разложении по собственным функциям диссипативных ядер", Докл. АН СССР, 99:4 (1954), 499-502.

[11] И. М. Глазман, "О разложимости по системе собственных элементов диссипативных операторов", УМН, 13:3(81) (1958), 179-181.

[12] М. Г. Крейн, "О признаках полноты системы корневых векторов диссипативного оператора", УМH, 14:3(87) (1959), 145-152; англ. пер.: M. G. Krein, "Criteria for completeness of the system of root vectors of a dissipative operator", Amer. Math. Soc. Transl. Ser. 2, 26, Amer. Math. Soc., Providence, RI, 1963, 221-229.

[13] В. Б. Лидский, "Условия полноты системы корневых подпространств у несамосопряженных операторов с дискретным спектром", Тр. ММО, 8, ГИФМЛ, М., 1959, 83-120; англ. пер.: V. B. Lidskii, "Conditions for completeness of a system of root subspaces for non-selfadjoint operators with discrete spectra", Amer. Math. Soc. Transl. Ser. 2, 34, Amer. Math. Soc., Providence, RI, 1963, 241-281.

[14] В. Б. Лидский, "О суммируемости рядов по главным векторам несамосопряженных операторов", Тр. ММО, 11, ГИФМЛ, М., 1962, 3-35; англ. пер.: V. B. Lidskii, "Summability of series in the principal vectors of non-selfadjoint operators", Amer. Math. Soc. Transl. Ser. 2, 40, Amer. Math. Soc., Providence, RI, 1964, 193-228.

[15] В. Б. Лидский, "О разложении в ряд Фурье по главным функциям несамосопряженного эллиптического оператора", Матем. сб., 57(99):2 (1962), 137-150.

[16] А. С. Маркус, "О базисе из корневых векторов диссипативного оператора", Докл. AH CCCP, 132:3 (1960), 524-527; англ. пер.: A. S. Markus, "A basis of root vectors of a dissipative operator", Soviet Math. Dokl., 1 (1960), 599-602.

[17] А.С. Маркус, "О разложении по корневым векторам слабо возмущенного самосопряженного оператора", Докл. АН СССР, 142:3 (1962), 538-541; англ. пер.: A. S. Markus, "Expansion in root vectors of a slightly perturbed self-adjoint operator", Soviet Math. Dokl., 3 (1962), 104-108.

[18] В.И. Мацаев, "Об одном классе вполне непрерывных операторов", Докл. АН CCCP, 139:3 (1961), 548-551; англ. пер.: V. I. Matsaev, "On a class of completely continuous operators", Soviet Math. Dokl., 2 (1961), 972-975.

[19] В.И. Мацаев, "Несколько теорем о полноте корневых подпространств вполне непрерывных операторов", Докл. АН СCCP, 155:2 (1964), 273-276; англ. пер.: V.I. Matsaev, "Some theorems on the completeness of root subspaces of completely continuous operators", Soviet Math. Dokl., 5 (1964), 396-399.

[20] В.И. Мацаев, "Об одном методе оценки резольвент несамосопряженных операторов", Докл. АН ССCP, 154:5 (1964), 1034-1037; англ. пер.: V.I. Matsaev, 
"A method for the estimation of the resolvents of non-selfadjoint operators", Soviet Math. Dokl., 5 (1964), 236-240.

[21] S. Agmon, "On the eigenfunctions and on the eigenvalues of general elliptic boundary value problems", Comm. Pure Appl. Math., 15 (1962), 119-147.

[22] S. Agmon, Lectures on elliptic boundary value problems, Van Nostrand Math. Stud., 2, D. Van Nostrand Co., Inc., Princeton, NJ-Toronto-London, 1965, v+291 pp.

[23] В. Э. Кацнельсон, О сходимости и суммируемости рядов по корневым векторам некоторых классов несамосопряженных операторов, Дисс. ... канд. физ.-матем. наук, Харьк. гос. ун-т, Харьков, 1967, 150 с.

[24] В.Э. Кацнельсон, “Об условиях базисности системы корневых векторов некоторых классов операторов", Функи. анализ и его прил., 1:2 (1967), 39-51; англ. пер.: V. È. Katsnel'son, "Conditions under which systems of eigenvectors of some classes of operators form a basis", Funct. Anal. Appl., 1:2 (1967), 122-132.

[25] И.Ц. Гохберг, М.Г. Крейн, Введение в теорию линейных несамосопряженных операторов в гилъбертовом пространстве, Наука, М., 1965, 448 с.; англ. пер.: I. C. Gohberg, M. G. Krein, Introduction to the theory of linear nonselfadjoint operators, Transl. Math. Monogr., 18, Amer. Math. Soc., Providence, RI, 1969, $\mathrm{xv}+378 \mathrm{pp}$.

[26] П. Д. Лакс, “Теорема Фрагмена-Линделефа в гармоническом анализе и ее приложения к некоторым вопросам теории эллиптических уравнений”, Математика. Сб. пер., 4, ИЛ, М., 1959, 107-132; пер. с англ.: Р. D. Lax, "A Phragmén-Lindelöf theorem in harmonic analysis and its application to some questions in the theory of elliptic equations", Comm. Pure Appl. Math., 10 (1957), 361-389.

[27] S. Agmon, L. Nirenberg, "Properties of solutions of ordinary differential equations in Banach space", Comm. Pure Appl. Math., 16 (1963), 121-239.

[28] А. С. Маркус, Введение в спектральную теорию полиномиальных операторных пучков, Штиинца, Кишинев, 1986, 260 с.; англ. пер.: А. S. Markus, Introduction to the spectral theory of polynomial operator pencils, Transl. Math. Monogr., 71, Amer. Math. Soc., Providence, RI, 1988, iv+250 pp.

[29] А. А. Шкаликов, “Эллиптические уравнения в гильбертовом пространстве и спектральные задачи, связанные с ними", Тр. сем. им. И. Г. Петровского, 14, Изд-во Моск. ун-та, М., 1989, 140-224; англ. пер.: А. A. Shkalikov, "Elliptic equations in Hilbert space and associated spectral problems", J. Soviet Math., 51:4 (1990), 2399-2467.

[30] А.С. Маркус, В.И. Мацаев, "Теорема о сравнении спектров и спектральная асимптотика для пучка М. В. Келдыша", Матем. сб., 123(165):3 (1984), 391-406; англ. пер.: А. S. Markus, V. I. Matsaev, "A theorem on comparison of spectra, and the spectral asymptotics for a Keldysh pencil", Math. USSR-Sb., 51:2 (1985), 389-404.

[31] A. A. Shkalikov, "Zeto distribution for pairs of holomorphic functions with applications to eigenvalue distribution", Trans. Amer. Math. Soc., 281:1 (1984), 49-63.

[32] Т. Като, Теория возмущений линейных операторов, Мир, М., 1972, 740 с.; пер. с англ.: Т. Kato, Perturbation theory for linear operators, Grundlehren Math. Wiss., 132, Springer-Verlag New York, Inc., New York, 1966, xix+592 pp.

[33] М. С. Агранович, "Спектральные свойства задач дифракции", Добавление к кн.: Н. Н. Войтович, Б. З. Каценеленбаум, А. Н. Сивов, Обобщенный метод собственных колебаний в теории дифракции, Наука, М., 1977, 289-416; перераб. англ. изд.: M. S. Agranovich, B. Z. Katsenelenbaum, A. N. Sivov, N. N. Voitovich, Generalized method of eigenoscillations in diffraction theory, Wiley-VCH Verlag Berlin GmbH, Berlin, 1999, Chapter 5.

[34] М. С. Агранович, "Спектральные задачи для сильно эллиптических систем второго порядка в областях с гладкой и негладкой границей”, УМH, 57:5(347) (2002), 3-78; англ. пер.: M. S. Agranovich, "Spectral problems for second-order strongly 
elliptic systems in smooth and non-smooth domains", Russian Math. Surveys, 57:5 (2002), 847-920.

[35] М. С. Агранович, "Спектральные задачи в липшицевых областях", Уравнения в частных производных, СМФН, 39, РУДН, М., 2011, 11-35; англ. пер.: M.S. Agranovich, "Spectral problems in Lipschitz domains", J. Math. Sci. (N. Y.), 190:1 (2013), 8-33.

[36] Г. В. Радзиевский, "Задача о полноте корневых векторов в спектральной теории оператор-функций”, УМН, 37:2(224) (1982), 81-145; англ. пер.: G. V. Radzievskii, "The problem of the completeness of root vectors in the spectral theory of operatorvalued functions", Russian Math. Surveys, 37:2 (1982), 91-164.

[37] Т. Я. Азизов, Н. Д. Копачевский, Приложения индефинитной метрики, Изд-во “ДИАЙПИ", Симферополь, 2014, 276 с.

[38] В. В. Власов, Д. А. Медведев, Н. А. Раутиан, "Функционально-дифференциальные уравнения в пространствах Соболева и их спектральный анализ", Современные проблемы математики и механики, 8, Изд-во Моск. ун-та, М., 2011, 308 с.

[39] B.S. Mityagin, "Spectral expansions of one-dimensional periodic Dirac operators", Dyn. Partial Differ. Equ., 1:2 (2004), 125-191.

[40] B. S. Mityagin, "The spectrum of a harmonic oscillator operator perturbed by point interactions", Internat. J. Theoret. Phys., 54:11 (2015), 4068-4085.

[41] J. Adduci, B. Mityagin, "Eigensystem of an $L^{2}$-perturbed harmonic oscillator is an unconditional basis", Cent. Eur. J. Math., 10:2 (2012), 569-589; 2010 (v1 - 2009), 28 pp., arXiv: 0912.2722.

[42] J. Adduci, B. Mityagin, "Root system of a perturbation of a selfadjoint operator with discrete spectrum", Integral Equations Operator Theory, 73:2 (2012), 153-175.

[43] П.Б. Джаков, Б. С. Митягин, “Зоны неустойчивости одномерных периодических операторов Шрёдингера и Дирака", УМH, 61:4(370) (2006), 77-182; англ. пер.: P. Djakov, B.S. Mityagin, "Instability zones of periodic 1-dimensional Schrödinger and Dirac operators", Russian Math. Surveys, 61:4 (2006), 663-766.

[44] P. Djakov, B. Mityagin, "Spectral gaps of Schrödinger operators with periodic singular potentials", Dyn. Partial Differ. Equ., 6:2 (2009), 95-165.

[45] P. Djakov, B. Mityagin, "Bari-Markus property for Riesz projections of 1D periodic Dirac operators", Math. Nachr., 283:3 (2010), 443-462.

[46] P. Djakov, B. Mityagin, "Convergence of spectral decompositions of Hill operators with trigonometric polynomial potentials", Math. Ann., 351:3 (2011), 509-540.

[47] P. Djakov, B. Mityagin, "Equiconvergence of spectral decompositions of 1D Dirac operators with regular boundary conditions", J. Approx. Theory, 164:7 (2012), 879-927.

[48] P. Djakov, B. Mityagin, "Criteria for existence of Riesz bases consisting of root functions of Hill and 1D Dirac operators", J. Funct. Anal., 263:8 (2012), 2300-2332.

[49] P. Djakov, B. Mityagin, "Riesz bases consisting of root functions of 1D Dirac operators", Proc. Amer. Math. Soc., 141:4 (2013), 1361-1375.

[50] B. Mityagin, P. Siegl, "Root system of singular perturbations of the harmonic oscillator type operators", Lett. Math. Phys., 106:2 (2016), 147-167; 2015, 16 pp., arXiv: $1307.6245 \mathrm{v} 2$.

[51] А. А. Шкаликов, "О базисности корневых векторов возмущенного самосопряженного оператора", Теория функций и дифференциальные уравнения, Сборник статей. К 105-летию со дня рождения академика Сергея Михайловича Никольского, Тр. МИАН, 269, 2010, 290-303; англ. пер.: А. A. Shkalikov, "On the basis property of root vectors of a perturbed self-adjoint operator", Proc. Steklov Inst. Math., 269 (2010), 284-298.

[52] A. A. Shkalikov, "Eigenvalue asymptotics of perturbed self-adjoint operators", Methods Funct. Anal. Topology, 18:1 (2012), 79-89; 2012, 12 pp., arXiv: 1202.5204. 
[53] Н. Данфорд, Дж. Т. Шварц, Линейные операторь. Спектральные операторь, Мир, М., 1974, 663 с.; пер. с англ.: N. Dunford, J. T. Schwartz, Linear operators. Part III. Spectral operators, Pure Appl. Math., 7, Interscience Publishers [John Wiley \& Sons, Inc.], New York-London-Sydney, 1971, i-xx and 1925-2592.

[54] А. С. Маркус, В. И. Мацаев, "Операторы, порожденные полуторалинейными формами, и их спектральные асимптотики", Линейные операторы и интегралъные уравнения, Матем. исслед., 61, Штиинца, Кишинев, 1981, 86-103.

[55] М. А. Красносельский, П. П. Забрейко, Е. И. Пустыльник, П. Е. Соболевский, Интегральные операторы в пространствах суммируемых функиий, Наука, М., 1966, 499 с.; англ. пер.: M. A. Krasnosel'skii, P. P. Zabreiko, E. I. Pustyl'nik, P. E. Sobolevskii, Integral operators in spaces of summable functions, Monographs and Textbooks on Mechanics of Solids and Fluids, Noordhoff International Publishing, Leiden, 1976, xv+520 pp.

[56] М. С. Агранович, А.М. Селицкий, “Дробные степени операторов, отвечающих коэрцитивным задачам в липшицевых областях", Функи. анализ и его прил., 47:2 (2013), 2-17; англ. пер.: M.S. Agranovich, A. M. Selitskii, "Fractional powers of operators corresponding to coercive problems in Lipschitz domains", Funct. Anal. Appl., 47:2 (2013), 83-95.

[57] Ф. Рисс, Б. Сёкефальви-Надь, Лекции по функииональному анализу, 2-е изд., Мир, М., 1979, 589 с.; пер. с фр.: F. Riesz, B. Sz.-Nagy, Leçons d'analyse fonctionnelle, 4ème éd., Gauthier-Villars, Paris; Akadémiai Kiadó, Budapest, 1965, viii+490 pр.; англ. пер. 2-го фр. изд.: F. Riesz, B. Sz.-Nagy, Functional analysis, Dover Books on Advanced Mathematics, Dover Publications, Inc., New York, 1990, xii+504 pp.

[58] А.С. Маркус, В.И. Мацаев, “Теоремы сравнения спектров линейных операторов и спектральные асимптотики", Тр. ММО, 45, Изд-во Моск. ун-та, М., 1982, 133-181; англ. пер.: A. S. Markus, V.I. Matsaev, "Comparison theorems for spectra of linear operators, and spectral asymptotics", Trans. Moscow Math. Soc., 1, Amer. Math. Soc., Providence, RI, 1984, 139-187.

[59] Х. Трибель, Теория интерполяиии, функциональные пространства, дифференциальные операторы, Мир, М., 1980, 660 с.; пер. с англ.: Н. Triebel, Interpolation theory, function spaces, differential operators, VEB Deutscher Verlag der Wissenschaften, Berlin, 1978, 528 pp.

[60] М. С. Агранович, "О рядах по корневым векторам операторов, определяемых формами с самосопряженной главной частью”, Функи. анализ и его прил., 28:3 (1994), 1-21; англ. пер.: M. S. Agranovich, "On series with respect to root vectors of operators associated with forms having symmetric principal part", Funct. Anal. Appl., 28:3 (1994), 151-167.

[61] М. Ш. Бирман, М. З. Соломяк, "Количественный анализ в теоремах вложения Соболева и приложения к спектральной теории", Десятая математическая школа (Кацивели-Нальчик, 1972), Ин-т матем. АН Укр. ССР, Киев, 1974, 5-189.

[62] М.Ш. Бирман, М. З. Соломяк, "Оценки сингулярных чисел интегральных операторов", УМH, 32:1(193) (1977), 17-84; англ. пер.: M. Sh. Birman, M. Z. Solomyak, "Estimates of singular numbers of integral operators", Russian Math. Surveys, 32:1 (1977), 15-89.

[63] Г. В. Розенблюм, М. З. Соломяк, М. А. Шубин, "Спектральная теория дифференциальных операторов", Дифференциальные уравнения с частными производными -7 , Итоги науки и техн. Сер. Соврем. пробл. матем. Фундам. направления, 64, ВИНИТИ, М., 1989, 5-242; англ. пер.: G. V. Rozenblum, M. A. Shubin, M.Z. Solomyak, "Spectral theory of differential operators", Partial differential equations VII, Encyclopaedia Math. Sci., 64, Springer, Berlin, 1994, 1-261.

[64] H. Weyl, "Inequalities between the two kinds of eigenvalues of a linear transformation", Proc. Nat. Acad. Sci. U. S. A., 35 (1949), 408-411. 
[65] B. Ya. Levin, Lectures on entire functions, Transl. Math. Monogr., 150, Amer. Math. Soc., Providence, RI, 1996, xvi+248 pp.

[66] G. D. Birkhoff, "On the asymptotic character of the solutions of certain linear differential equations containing a parameter", Trans. Amer. Math. Soc., 9:2 (1908), 219-231.

[67] G. D. Birkhoff, "Boundary value and expansion problems of ordinary differential equations", Trans. Amer. Math. Soc., 9:4 (1908), 373-395.

[68] М.С. Агранович, М.И. Вишик, "Эллиптические задачи с параметром и параболические задачи общего вида", УМН, 19:3(117) (1964), 53-161; англ. пер.: M.S. Agranovich, M.I. Vishik, "Elliptic problems with a parameter and parabolic problems of general type", Russian Math. Surveys, 19:3 (1964), 53-157.

[69] М.А. Наймарк, Линейные дифференииальные операторы, 2-е изд., Наука, М., 1969, 526 с.; англ. пер. 1-го изд.: М. А. Naimark, Linear differential operators, v. I, II, Frederick Ungar Publishing Co., New York, 1967, 1968, xiii+144 pp., xv+352 pp.

[70] A. Shkalikov, "Estimates of meromorphic functions and summability theorems", $P a$ cific J. Math., 103:2 (1982), 569-582.

[71] А. А. Шкаликов, "Об оценках мероморфных функций и суммировании рядов по корневым векторам несамосопряженных операторов", Докл. АН CCCP, 268:6 (1983), 1310-1314; англ. пер.: А. A. Shkalikov, "On estimates of meromorphic functions and summation of series in the root vectors of nonselfadjoint operators", Soviet Math. Dokl., 27 (1983), 259-263.

[72] А. А. Шкаликов, “Теоремы тауберова типа о распределении нулей голоморфных функций”, Матем. сб., 123(165):3 (1984), 317-347; англ. пер.: А. A. Shkalikov, "Theorems of Tauberian type on the distribution of zeros of holomorphic functions", Math. USSR-Sb., 51:2 (1985), 315-344.

[73] А. С. Маркус, В. И. Мацаев, "О сходимости разложений по собственным векторам оператора, близкого к самосопряженному", Линейные операторы и интегральные уравнения, Матем. исслед., 61, Штиинца, Кишинев, 1981, 104-129.

[74] М. С. Агранович, "О сходимости рядов по корневым векторам операторов, близких к самосопряженным", Тр. ММО, 41, Изд-во Моск. ун-та, М., 1980, 163-180; англ. пер.: M.S. Agranovich, "On the convergence of series in the root vectors of almost selfadjoint operators", Trans. Mosc. Math. Soc., 1982, № 1, Amer. Math. Soc., Providence, RI, 1982, 167-182.

[75] Дж. Гарнет, Ограниченные аналитические функиии, Мир, М., 1984, 470 с.; пер. с англ.: J.B. Garnett, Bounded analytic functions, Pure Appl. Math., 96, Academic Press, Inc. [Harcourt Brace Jovanovich, Publishers], New York-London, 1981, xvi+467 pp.

[76] B.S. Mityagin, P. Siegl, Local form-subordination condition and Riesz basisness of root systems, 2016, 28 pp., arXiv: 1608.00224.

[77] Г. Г. Харди, Дж. И. Литтльвуд, Г. Полиа, Неравенства, ИЛ, М., 1948, 456 с.; пер. с англ.: G. H. Hardy, J. E. Littlewood, G. Pólya, Inequalities, Univ. Press, Cambridge, 1934, xii+314 pp.

[78] N. Dunford, "A survey of the theory of spectral operators", Bull. Amer. Math. Soc., 64:5 (1958), 217-274.

[79] В. П. Михайлов, "О базисах Рисса в $\mathscr{L}_{2}[0,1] ”$, Докл. АН CCCP, 114:5 (1962), 981-984; англ. пер.: V.P. Mikhailov, "Riesz bases in $\mathscr{L}_{2}[0,1]$ ", Soviet Math. Dokl., 3 (1962), 851-855.

[80] Г. М. Кесельман, "О безусловной сходимости разложений по собственным функциям некоторых дифференциальных операторов”, Изв. вузов. Матем., 1964, № 2, 82-93.

[81] А. А. Шкаликов, "О базисности собственных функций обыкновенного дифференциального оператора", УМH, 34:5(209) (1979), 235-236; англ. пер.: A. A. Shkalikov, 
"On the basis problem of the eigenfunctions of an ordinary differential operator", Russian Math. Surveys, 34:5 (1979), 249-250.

[82] А.А. Шкаликов, "О свойстве базисности собственных функций обыкновенных дифференциальных операторов с интегральными краевыми условиями”, Вестн. Моск. ун-та. Сер. 1 Матем. Мех., 1982, №6, 12-21; англ. пер.: А. A. Shkalikov, "The basis problem of the eigenfunctions of ordinary differential operators with integral boundary conditions", Moscow Univ. Math. Bull., 37:6 (1982), 10-20.

[83] А. А. Шкаликов, "Краевые задачи для обыкновенных дифференциальных уравнений с параметром в граничных условиях", Тр. сем. им. И.Г. Петровского, 9, Изд-во Моск. ун-та, М., 1983, 190-229; англ. пер.: А. A. Shkalikov, "Boundary problems for ordinary differential equations with parameter in the boundary conditions", J. Soviet Math., 33 (1986), 1311-1342.

[84] А.М. Савчук, А.А. Шкаликов, "Операторы Штурма-Лиувилля с потенциалами-распределениями", Тр. ММО, 64, УРСС, М., 2003, 159-212; англ. пер.: A. M. Savchuk, A.A. Shkalikov, "Sturm-Liouville operators with distribution potentials", Trans. Moscow Math. Soc., 2003, 2003, 143-192.

[85] A. M. Savchuk, A. A. Shkalikov, "The Dirac operator with complex valued summable potential", Math. Notes, 95:5 (2014), 777-810; рус. пер.: А. М. Савчук, А. А. Шкаликов, Оператор Дирака с комплекснозначным суммируемым потенииалом, 2014, 34 c., arXiv: 1412.6757.

[86] А.А. Лунев, М. М. Маламуд, "О базисности Рисса системы корневых векторов для $(2 \times 2)$-системы типа Дирака", Докл. РАН, 458:3 (2014), 255-260; англ. пер.: A. A. Lunyov, M. M. Malamud, "On the Riesz basis property of the root vector system for Dirac-type $2 \times 2$ systems", Dokl. Math., 90:2 (2014), 556-561.

[87] A. A. Lunyov, M. M. Malamud, "On the Riesz basis property of root vectors system for $2 \times 2$ Dirac type operators", J. Math. Anal. Appl., 441:1 (2016), 57-103.

[88] И. В. Садовничая, "Равносходимость спектральных разложений для системы Дирака с потенциалом из пространств Лебега", Функциональные пространства, теория приближений, смежные разделы математического анализа, Сборник статей. K 110-летию со дня рождения академика Сергея Михайловича Никольского, Тр. МИАН, 293, МАИК, М., 2016, 296-324; англ. пер.: I. V. Sadovnichaya, "Equiconvergence of spectral decompositions for the Dirac system with potential in Lebesgue spaces", Proc. Steklov Inst. Math., 293 (2016), 288-316.

[89] И.В. Садовничая, " $L_{\mu} \rightarrow L_{\nu}$ равносходимость спектральных разложений для системы Дирака с $L_{\varkappa}$ потенциалом", 2015, 6 с., arXiv: 1512.02021.

\section{Андрей Андреевич Шкаликов}

Поступила в редакцию

(Andrei A. Shkalikov)

25.07 .2016

Московский государственный университет

им. М. В. Ломоносова

E-mail: shkalikov@mi.ras.ru 